\title{
3 Ist Meeting of the European Association for the Study of Diabetes Eye Complications Study Group (EASDec) Odense, Denmark, 28th - 30th October 2021
}

European Journal of Ophthalmology $|-4|$

(C) The Author(s) 2021 Article reuse guidelines: sagepub.com/journals-permissions DOI: $10.1177 / 1 \mid 20672$ |2।104703। journals.sagepub.com/home/ejo

(S)AGE

\section{Topical Ocular Administration of Sitagliptin, a DPP-4 Inhibitor, Prevents the Downregulation of Neuroretinal Presynaptic Proteins Induced by Diabetes}

C. Hernández, P. Bogdanov, H. Ramos, D. Sabater, J. Huerta, R. Simó

Diabetes and Metabolism Research Unit and CIBERDEM, Vall d'Hebron Research Institute, Barcelona, Spain

DESIGN. Experimental study

PURPOSE. The increase in the number of patients with diabetes resulted in diabetic retinopathy (DR) becoming a global health concern. This is mainly due to the unsatisfactory therapeutic strategies addressing the early stages of the disease, in which the impairment of neurovascular unit plays an essential role. Neurovascular unit comprises a functional coupling between blood vessels, glia and neurons, that coordinates several physiological functions such as metabolic demand, blood delivery or synaptic activity. Neurovascular impairment leads to microvascular abnormalities and neurodegeneration, where synapse damage plays a key role. Reduced synaptic protein expression, impaired neurotransmission and alterations in neuronal morphology have been observed in retinas from several diabetic animal models. We previously reported that the topical administration (eye drops) of sitagliptin, a dipeptidyl peptidase-4 (DPP-4) inhibitor, prevented retinal neurodegeneration induced by diabetes in $\mathrm{db} / \mathrm{db}$ mice. The aim of this study is to examine whether the modulation of presynaptic proteins is a mechanism involved in the neuroprotective effect of sitagliptin.

METHODS. A total of $15 \mathrm{db} / \mathrm{db}$ mice, aged 12 weeks, received a topical administration of sitagliptin $(5 \mu \mathrm{L}$; concentrations: $10 \mathrm{mg} / \mathrm{mL}$ ) twice per day for 2 weeks while other $15 \mathrm{db} / \mathrm{db}$ were treated with vehicle $(5 \mu \mathrm{L})$. Fifteen non-diabetic mice $(\mathrm{db} /+)$ were used as a control group. Protein levels were assessed through western blotting and immunohistochemistry (IHC), and mRNA levels were evaluated through reverse transcription polymerase chain reaction (RT-PCR).

RESULTS. Our results revealed a down-regulation (protein and mRNA levels) of several presynaptic proteins such as syntaxin 1A, synapsin I, synaptotagmin, synaptophysin, vesicle-associated membrane protein 2 (vamp2) and synaptosomal-associated protein of $25 \mathrm{kDa}$ (SNAP25) in diabetic mice treated with vehicle. These proteins are involved in vesicle biogenesis, mobilization and docking, membrane fusion and recycling, and synaptic neurotransmission. Sitagliptin was able to significantly prevent the down-regulation of these proteins.

CONCLUSIONS. We conclude that sitagliptin exerts beneficial effects in the retinas of $\mathrm{db} / \mathrm{db}$ mice by preventing the downregulation of crucial presynaptic proteins. These neuroprotective effects open a new avenue for treating DR as well other retinal diseases in which neurodegeneration/ synaptic abnormalities play a relevant role.

\section{Characterization of a New Immortalized Human Microglial Cell Line and its Pro-Inflammatory Activation}

A. Mazzeo, E. Beltramo, M. Porta

Department of Medical Sciences, University of Torino, Turin, Italy

DESIGN. Experimental

PURPOSE. The complexity of retinal structure reflects on the difficulty to describe its composite cell interactions and the pathways involved. Microglial cells are the main responsible cell-type for the modulation of immune responses to inflammatory stimuli that occur in the retina in diabetes. The majority of studies on retinal inflammation in diabetic retinopathy in the literature use rodent microglial lines, because of the scarce availability of human cell sources. We carried out a detailed characterization of a 
new commercially available immortalized human microglial line (Innoprot, Spain), and tested its susceptibility to inflammatory stimuli, to create species-specific cell models to study the multiple interactions between the vascular and neuronal portions of the retina.

METHODS. First, we checked the expression of microglial markers, capable of discriminating between resident microglia and peripheral macrophages (TMEM119, Iba-1, CD14, CD16, CD68), and human vs rodent origin (CD11b). Subsequently, we tried LPS stimulation as well as several different pro-inflammatory cocktails to determine the best combination able to induce a significant M1 (inflammatory) response after $24 \mathrm{~h}$ exposure. We then measured M1 induction through the modulation of the expression of pro- and anti-inflammatory molecules, determined by flow cytometry, Western blotting, and RT-PCR. Finally, we performed morphology and functional assays, measuring viability, apoptosis, migration and ROS production.

RESULTS. Marker expression confirmed the microglial derivation of the cells and their human origin. Differently from rodents, LPS is not able to induce an M1 profile in human microglial cells. The best pro-inflammatory combination among those tested was $20 \mathrm{ng} / \mathrm{ml} \mathrm{hIL-1} \beta+10$ $\mathrm{ng} / \mathrm{ml} \mathrm{hTNF} \alpha+50 \mathrm{ng} / \mathrm{ml}$ hINF $\gamma$. This was able to induce changes in morphology, resulting in elongation and ramification, characteristic of activated cells, and to increase proliferation, apoptosis, migration, and ROS production. In addition, M1 conditions was associated with increased expression of inflammatory markers: IL-6, IL-8, MMP2, VCAM-1, TNF $\alpha$, MHC-II, and pNFK $\beta / \mathrm{NFK} \beta$, and the pro-inflammatory miR-155 and miR146a.

CONCLUSIONS. This immortalized human microglial line proved to be a potential experimental tool to investigate the pathophysiology of the inflammatory component of diabetic retinopathy in species-specific models.

\section{Towards a Better Understanding of PLVAP in Vascular Permeability: A Proteomic Approach to Identify Interacting Partners of PLVAP}

\author{
E. Bosma ${ }^{1,2}$, E.K. Bosma ${ }^{1,2}$, C.J.F. Van Noorden², R.O. \\ Schlingemann', I. Klaassen ${ }^{1,2}$ \\ 'Department of Ophthalmology, Amsterdam UMC, The Netherlands \\ ${ }^{2}$ Department of Medical Biology, Amsterdam UMC, The Netherlands
}

DESIGN. Fundamental research study with primary endothelial cells

PURPOSE. Current treatment options for diabetic macular edema (DME) remain suboptimal, necessitating the development of new treatment options. The protein plasmalemma vesicle-associated protein (PLVAP) has emerged as a new potential therapeutic target for DME. PLVAP expression is absent from healthy inner blood-retinal barrier blood vessels, but its expression is induced in blood vessels of patients with diabetic retinopathy where it coincides with increased vascular leakage. PLVAP localizes to vesicular structures called caveolae in continuous endothelium, where it forms a sieve structure in the neck of the caveolae. Our aim is to better understand the molecular mechanism of PLVAP in vascular permeability, and identify interacting proteins, which is essential before antiPLVAP therapies can be developed.

METHODS. Experiments were performed with human umbilical vein endothelial cells (HUVECs) and essential experiments will be repeated with retinal endothelial cells. PLVAP tagged with a small 3xHA-tag was overexpressed in HUVECs by lentiviral transduction, and subsequently PLVAP enriched fractions were isolated by sucrose gradient ultracentrifugation in a Triton X-100 buffer. We aim to pull down PLVAP and its interacting partners from this sample, and analyze all associated proteins with tandem mass spectrometry. We will confirm the binding of these proteins to PLVAP by immunoprecipitation, confocal imaging and immuno-electron microscopy.

RESULTS. When overexpressing the PLVAP-3xHA construct in HUVECs, a punctuate localization pattern for this construct was detected with confocal imaging, indicative of vesicular structures. Specific localization of PLVAP$3 \mathrm{xHA}$ to caveolae was confirmed by immuno-electron microscopy. Enriched protein fractions of endogenous PLVAP were successfully obtained by sucrose density gradient ultracentrifugation. A subpopulation was found to be located in the same fraction as caveolin-1, the main component of caveolae, whereas the majority was found in the lighter density fractions. Experiments with retinal endothelial cells that overexpress PLVAP-3XHA are still ongoing.

CONCLUSIONS. PLVAP tagged with a small $3 \mathrm{xHA}$ tag seems to localize to correct subcellular structures in endothelial cells, and can therefore be used for further experiments in order to identify the interacting partners of PLVAP.

\section{Immunomodulation of Microglia/Macrophage Interaction and its Effect in Diabetic Retinopathy Progression}

F. Cano-Cano',2, L. Gomez-Jaramillo',2, M.C. GonzalezMontelongo',2, E. Zubia ${ }^{3}$, M.J. Ortega ${ }^{3}$, M. Aguilar-Diosdado 1,2, A.I. Arroba ${ }^{1,2}$

'Research Unit, Instituto de Investigación e Innovación en Ciencias Biomédicas de la Provincia de Cádiz (INiBICA), University Hospital "Puerta del Mar", Cádiz, Spain

${ }^{2}$ Department of Endocrinology and Metabolism, Instituto de Investigación e Innovación en Ciencias Biomédicas de la Provincia de Cádiz (INiBICA), University Hospital "Puerta del Mar", Cádiz, Spain

${ }^{3}$ Departamento de Química Orgánica, Facultad de Química, Universidad de Cádiz, Cádiz, Spain 
DESIGN. Retinal inflammation occurs during an early stage in diabetic retinopathy (DR), which is believed to play a crucial role in the development and progression of DR. Algae bioactive extracts have shown properties against inflammatory processes. The present study analyses of microglia/macrophage dynamics in retinal diseases influenced by systemic inflammation, rendering the regulation of these cells activation a potentially important therapeutic target for DR

PURPOSE. The aim of this work was to investigate the effects of the biological molecules on inflammation associated with early diabetic retinopathy by immunomodulation of specific immune retinal system and the possible role of systemic immune cells in DR progression.

METHODS. Bv.2 and Raw264.7 cells were stimulated with lypopolysaccharide (LPS), as an inflammatory stimulus and/or bioactive molecules. The effects of conditioned medium (CM) from Raw264.7 on Bv.2 cells were analysed in order to determine the contribution of both immune system in DR progression and immuno-modulation. Retinal explants from BB rat, at 7 weeks was cultured in the presence or absence of bioactive molecules, and with both CM from Bv.2 or Raw264.7 cells. Pro/anti-inflammatory and signalling pathways were analysed by qPCR, Western blotting and immunofluorescence

RESULTS. In a prediabetic status (normo-glycaemia) of T1DM animal model, we found retinal inflammatory events that antecedes to hyperglycaemic detection. Bv.2 microglial, Raw264.7 macrophage cells cultured under diabetic environment and retinal explants from $\mathrm{BB}$ rats treated with bioactive molecules reduces the pro-inflammatory markers, such as Nos2, Il1b, I16 and Tnfa expression. In retinal explants from $\mathrm{BB}$ rats normo-glycemic, the activation of the inflammasome complex is blocked and bioactive molecules is able to induce Arginase-1 expression (M2 response). The CM from macrophage cells cultured in diabetic environment in presence or absence of bioactive molecules induced an immune differential response on microglial cells and retinal explants from wild type rat

CONCLUSIONS. The inflammatory processes that precedes to DR is able to be modulated by algae bioactive molecules, which exerts an anti-inflammatory effect on retinal and systemic immune cells that and could be an effective alternative for DR treatment and/or prevention.

\section{Mapping the Daily Rhythmic Transcriptome in the Diabetic Retina}

E. Beli, H.R. Winter, R. Silk, V. Tiwari, D Simpson, A. Stitt

Wellcome-Wolfson Institute of Experimental Medicine, Queen's University Belfast, Belfast, UK

DESIGN. Herein we mapped how diabetes affects the daily rhythmic transcriptome in the mouse retina using deep mRNA sequencing methods.
PURPOSE. The eye is a rhythmic organ specifically evolved to function around the light cycle via functional circadian clocks. Diseases such as diabetes have been reported to disrupt circadian rhythms and circadian disruption emerges as an important factor in the prognosis of disease outcomes and treatment success. Our purpose was to understand the extent of circadian disruption in the mouse retina due to diabetes.

METHODS. Healthy control and Ins2Akita/J diabetic mice were kept under a physiological $12 \mathrm{~h}: 12 \mathrm{~h}$ light-dark cycle until 4 months of age. Deep mRNA sequencing was conducted in retinas collected every 4 hours around the day/night cycle. Computational approaches were used for detection of rhythmicity, acrophase prediction, differential rhythmic patterns, phase set enrichment analysis and upstream regulator predictions.

RESULTS. Almost $10 \%$ of the retinal transcriptome was identified as rhythmic with a clear $12 \mathrm{hr}$ axis of transcriptional activity, peaking at midday and midnight. Although the 12-hour transcriptional axis is retained in the diabetic retina, it was phase advanced by approximately 1-3 hours. Downstream analysis identified oxygen sensing mechanisms and HIF1A as major predicted upstream regulators. In vitro experiments with hypoxia indicated that indeed hypoxia can phase shift the clock in human retinal endothelial cells.

CONCLUSIONS. To our knowledge this is the first study mapping the effects of diabetes in the rhythmic output in the retina. Importantly, we identified that hypoxia may be a primary driver of disrupted circadian rhythms in the diabetic retina.

\section{Circadian Clocks in Diabetic Retinal Endothelial Cells}

H.R. Winter, D. Hughes, A. Margariti, A.W. Stitt, E. Beli

Wellcome-Wolfson Institute for Experimental Medicine, Queen's University Belfast, Belfast, UK

DESIGN. An in vitro primary cell model was used to examine how hyperglycaemia and hypoxia affected the circadian clock in retinal endothelial cells. Cells were synchronised with $50 \%$ serum shock and repeated samples collected every two hours over 36 hours.

PURPOSE. Diseases such as diabetes have been reported to disrupt circadian rhythms and circadian disruption emerges as an important factor in the prognosis of disease outcomes and treatment success. Our objective was to investigate whether diabetes affects circadian gene expression in endothelial cells and the mechanisms involved.

METHODS. Induced Pluripotent Stem Cell-Derived Endothelial Cells (iPS-ECs) from healthy and diabetic patients were sequenced and differential analysis was performed. Genes related to circadian rhythms were identified. 
Primary human retinal endothelial cells (HRECs) were cultured in vitro in hyperglycaemic and hypoxic conditions to validate results. Circadian gene expression measurement was performed using RT-PCR.

RESULTS. iPS-ECs from diabetic patients had 5.7 fold reduction in Dec 2 mRNA expression, and 4.0 fold increase in Bmal-2. Synchronised HRECs under hypoxic conditions showed more robust circadian oscillation but lower amplitude of Bmal-1 and Cry1 mRNA expression, indicating an effect of hypoxia on circadian rhythmicity. Four weeks of hyperglycaemic conditions resulted in a slight increase of Bmal-1 and reduction in Cry1, Cry2 mRNA expression. Dec2 was the gene most affected by hyperglycaemic conditions. Hypoxia had a significant effect in reducing the expression of the majority of circadian clock genes.

CONCLUSIONS. Diabetic conditions resulted in a specific reduction of Dec2 expression in both patient derived iPS-ECs and HRECs in hyperglycaemic conditions. Hypoxia alone had more pronounced effects on circadian gene expression and rhythmicity compared to hyperglycaemia alone.

\section{Five-Year Incidence and Progression of Age- Related Macular Degeneration in People with Diabetes}

D. Romdhoniyyah', C.P. Cheyne ${ }^{4}$, A. Alshukri', D.G. Parry 3 , M.G. Finana ${ }^{4}$, S.P. Harding ${ }^{1,2,3}$, N.A.V. Beare ${ }^{1,2}$

'Department of Eye and Vision Science, Institute of Life Course and Medical Sciences, University of Liverpool, Member of Liverpool Health Partners, Liverpool, UK

${ }^{2}$ St. Pauls Eye Unit, Liverpool University Hospitals NHS Foundation Trust, Member of Liverpool Health Partners, Liverpool, UK

${ }^{3}$ Liverpool Ophthalmic Reading Centre, University of Liverpool and Liverpool University Hospitals NHS Foundation Trust, Liverpool, UK

${ }^{4}$ Department of Health Data Science, Institute of Population Health,

University of Liverpool, Member of Liverpool Health Partners, Liverpool, UK

DESIGN. Retrospective study of routinely collected fundus photographs.

PURPOSE. Age-related macular degeneration (AMD) and diabetic retinopathy (DR) increasingly occur simultaneously as the prevalence of diabetes rises in people over 50. Evidence on any pathophysiological relationship is conflicting. We investigated the association between AMD and DR by study the 5 -year incidence and progression of AMD in people with diabetes (PWD) attending a DR screening program.

METHODS. 1,500 people aged $\geqslant 50$ years were randomly selected from 10,336 PWD who attended the Liverpool Diabetic Eye Screening Programme in 2011. Retinal images and clinical information (estimated diabetes duration, $\mathrm{HbA1c}$, lipids, smoking status) were retrieved at baseline and in year 5. AMD grading followed the modified Age-Related Eye Disease Study system; non-central geographic atrophy was assigned to level 4 (late AMD). DR was graded within routine screening by trained accredited graders following national standards. Multiple logistic regression with multiple imputation adjusting for possible confounding factors was used for data analysis; variables were discounted if missing values were $\geqslant 20 \%$.

RESULTS. 1,492 of the 1,500 PWD had gradable retinal photographs at baseline. 258/1,492 had ungradable/unavailable images of both eyes in year 5 leaving 1,234 sets of photographs for analysis. The incidences of early, intermediate, late and any AMD were $12.1 \%, 11.5 \%, 2.2 \%$, and $15.6 \%$. Progression from early/intermediate to late AMD occurred in $11.4 \%$. Progression of any AMD (increase in $\geqslant 1$ AMD stages) occurred in $30.3 \%$.

In multiple logistic regression analysis, increasing age was associated with increased risk of incidence of intermediate (OR 1.05; 95\%CI 1.02-1.07, $\mathrm{p}<0.001)$, late $(1.16 ; 1.08$ $1.25, \mathrm{p}<0.001)$, and any AMD $(1.03 ; 1.01-1.05, \mathrm{p}=0.003)$, as expected. Likewise, increasing age was related to late $(1.13 ; 1.03-1.24, \mathrm{p}=0.01)$ and any AMD progression (1.04; $1.02-1.05, \mathrm{p}<0.001)$. No significant association was identified between the presence or severity of DR, diabetes duration, glycaemic/lipid control or smoking status at baseline with AMD incidence or progression (all $\mathrm{p} \geqslant 0.05$ ).

CONCLUSIONS. We did not find any evidence that DR is protective or is a risk factor for AMD incidence or progression over a 5 year timeframe. This suggests the mechanisms of these prevalent retinal diseases may not shared.

\section{Relationship between Diabetic Retinopathy and Systemic Neurodegenerative Diseases: A Systematic Review and Meta-Analysis}

J. Grauslund ${ }^{1,2,3}$, H.E. Pedersen ${ }^{1,2}$, C.H. Sandvik ${ }^{1,2}$, Y. Subhi ${ }^{4,5}$, F.N. Pedersen ${ }^{1,2}$

'Department of Ophthalmology, Odense University Hospital, Odense, Denmark

${ }^{2}$ Department of Clinical Research, University of Southern Denmark, Odense, Denmark

${ }^{3}$ Steno Diabetes Center Odense, Odense University Hospital, Odense,

Denmark

${ }^{4}$ Department of Ophthalmology, Zealand University Hospital, Roskilde, Denmark

${ }^{5}$ Department of Ophthalmology, Rigshospitalet, Glostrup, Denmark

DESIGN. Systematic review and meta-analysis.

PURPOSE. In recent years, retinal neurodegeneration has been identified as an early event in diabetic retinopathy (DR). To examine the potential role of systemic neurodegeneration, this study aimed to provide an overview of the available evidence on the relationship between DR and systemic neurodegeneration and to provide summary estimates on the measures of association.

METHODS. We searched the literature databases PubMed/MEDLINE, EMBASE, and the Cochrane Library 
on 3 October 2020 for all observational studies on humans evaluating the association between DR and systemic neurodegenerative diseases. Two authors conducted literature search, study selection, and data extraction in an independent fashion. Studies were reviewed qualitatively in text and quantitatively in meta-analyses.

RESULTS. We identified 27 eligible studies with a total of $1,398,041$ patients with diabetes. Diagnosis of DR was made using fundus photography or examination $(n=20)$, health registries $(n=4)$, self-reported $(n=1)$, and not disclosed in the remaining studies. Neurodegenerative conditions studied were cognitive impairment $(\mathrm{n}=23)$, Alzheimer's disease $(n=3)$, and Parkinson's disease $(n=1)$. In cross-sectional and longitudinal studies, respectively, presence of any DR was associated with present (OR: 1.57; $95 \%$ CI: 1.02 to $2.43, \mathrm{P}=0.043$ ) and incident (OR: 1.68 ; $95 \%$ CI: 1.25 to $2.27, \mathrm{P}=0.00066$ ) systemic neurodegeneration, but severity of DR was not associated with differences in systemic neurodegeneration (OR: $0.98 ; 95 \%$ CI: 0.45 to $2.15, \mathrm{P}=0.96$ ).

CONCLUSIONS. In this systematic review, we identified DR as a potential marker of systemic neurodegeneration. Further studies are warranted to better elucidate the clinical practical implications of this relationship.

\section{Non-Attendance at Diabetic Eye Screening in the Danish Nationwide Screening Program}

A.S. Thykjær ${ }^{1,4}$, L. Stokholm ${ }^{4}$, R. Kawasaki ${ }^{5}$, K. Højlund ${ }^{2,3}$, K.H. Rubin ${ }^{4}$, J. Grauslund ${ }^{1,2,3}$, on behalf of the OASIS Study Group

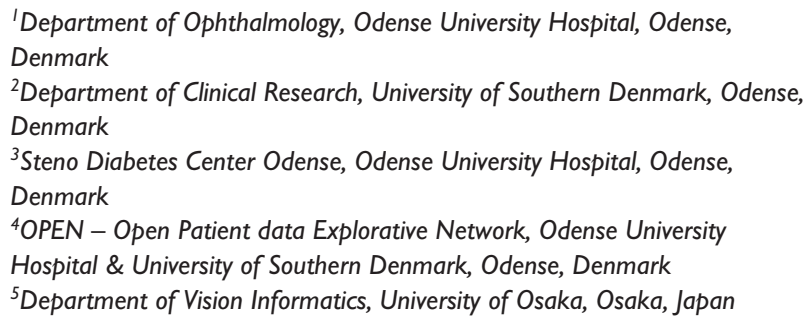

'Department of Ophthalmology, Odense University Hospital, Odense, Denmark

${ }^{2}$ Department of Clinical Research, University of Southern Denmark, Odense, Denmark

${ }^{3}$ Steno Diabetes Center Odense, Odense University Hospital, Odense, Denmark

${ }^{4}$ OPEN - Open Patient data Explorative Network, Odense University Hospital \& University of Southern Denmark, Odense, Denmark

${ }^{5}$ Department of Vision Informatics, University of Osaka, Osaka, Japan

DESIGN. Registry based national cohort study

PURPOSE. In Denmark, a nationwide screening program for diabetic retinopathy (DR) has been established since 2013. Patients with diabetes are, free of charge, offered regular diabetic eye screenings by practicing ophthalmologists or at selected hospital departments.

The aim of this study was to perform a real-life national evaluation of the DR screening program in Denmark, examining the screening pattern and the ability to remain within the screening program.

METHODS. The study population consisted of a longitudinal, nationwide cohort of diabetes patients $(n=206,220)$ in Denmark, who participated in the nationwide screening program for DR from 2013 through 2018 and were registered in the Danish Registry of Diabetic Retinopathy
(DiaBase) database. Data from DiaBase was enriched with demographic data from the Danish Civil Registration register and comorbidity information from The Danish National Patient Register.

We investigated a sub cohort of 28,990 patients in DiaBase with unexplained non-attendance after first diabetic eye screening. Patients, who died, migrated, disappeared, or were not due for next screening episode within the observation period, were excluded.

RESULTS. The baseline prevalence of DR in non-attendees was $9.3 \%$ as compared to $16.0 \%$ in repeating attendees $(\mathrm{p}<0.001)$.

There was a geographical skewing in the fraction of nonattendees in the five Danish regions; ranging from $9.4 \%$ in the Region of Southern Denmark to $18.4 \%$ in the Capital Region of Denmark $(p<0.001)$.

Non-attendees did not differ from the repeating attendees with regards to age, gender, marital status or screening facility (private practicing ophthalmologist or hospital), likewise they had an equivalent Charlson comorbidity score, as compared to repeating attendees.

CONCLUSIONS. In a real-life, national diabetic eye screening program from Denmark, $14.1 \%$ of patients, without evident reason, only attended eye screening once. Data suggests that non-attendance might be related to geographical factors and lower DR severity, as opposed to general comorbidity. Technical differences between the Danish regions might cause variations in registrations from examining doctors.

\section{Association between Diabetic Retinopathy and Glaucoma: Results from a National Screening Program. A National Study}

S. Sperling', L. Stokholm 2,4 J. Andresen ${ }^{5}$, M. Kolko ${ }^{6,7,8}$, A.H. Vestergaard', J. Grauslund ',2,3

'Department of Ophthalmology, Odense University Hospital, Odense, Denmark

${ }^{2}$ Department of Clinical Research, University of Southern Denmark, Odense, Denmark

${ }^{3}$ Steno Diabetes Center Odense, Odense University Hospital, Odense, Denmark

${ }^{4}$ OPEN - Open Patient data Explorative Network, Odense University Hospital \& University of Southern Denmark, Odense, Denmark

${ }^{5}$ Organization of Danish Practicing Ophthalmologists, Copenhagen, Denmark

${ }^{6}$ Department of Ophthalmology, Rigshospitalet-Glostrup, Copenhagen,

Denmark

${ }^{7}$ Department of Clinical Medicine, University of Copenhagen, Copenhagen, Denmark

${ }^{8}$ Department of Drug Design and Pharmacology, University of Copenhagen, Denmark

DESIGN. A cross-sectional and bi-directional 5-year prospective, registry-based cohort study.

PURPOSE. While previous smaller studies have proposed an association between diabetic retinopathy (DR) and glaucoma, longitudinal data from larger cohorts are 
lacking. We aimed to investigate if DR and glaucoma are cross-sectionally and longitudinally linked.

METHODS. Data of exposure and outcome were extracted from national registers, including the Danish Registry of Diabetic Retinopathy (DiaBase), the Danish Civil Registration System, the Danish National Patient Register and the Danish National Prescription Registry. We included 205,970 persons with diabetes from DiaBase (cases), who had attended diabetic eye screening and 1,003,170 age- and gender-matched non-diabetes controls. Exposures were (i) present and (ii) level-specific DR, and (iii) glaucoma. Outcomes were multivariable adjusted odds ratio (OR) for prevalent glaucoma (i) and multivariable adjusted hazard ratios (HR) for 5-year incident glaucoma (ii) and DR (iii). According to the International Clinical Retinopathy Disease Severity Scale, the level of DR was defined by the highest level of the two eyes. Glaucoma was defined by diagnostic codes $\left(\mathrm{H} 40^{*}\right)$ or at least three redeemed prescriptions of glaucoma medication $\left(\mathrm{S}_{01 E^{*}}\right)$ within one year.

RESULTS. At the index date, the prevalence of glaucoma was $4.7 \%$ for cases $(n=9,584)$ and $3.3 \%$ for controls $(n=32,746)$. Compared to controls, cases had a higher prevalence of glaucoma (multivariable adjusted OR 1.30, 95\% CI 1.27-1.34), which increased with higher levels of DR (multivariable adjusted OR 1.18 vs. 1.52 vs. 1.34 vs. 2.69 vs. 6.06 for DR levels $0-4$, respectively). Compared to controls, cases were also more likely to develop glaucoma (multivariable adjusted HR 1.12, 95\% CI 1.071.17). Compared to cases without glaucoma, cases with glaucoma were more likely to develop DR (multivariable adjusted HR 1.12, 95\% CI 1.02-1.22).

CONCLUSIONS. In this national cohort study, persons with diabetes were $30 \%$ more likely to have been diagnosed with glaucoma, with an increasing risk for persons with DR. Persons with diabetes had a $12 \%$ higher risk to be diagnosed with glaucoma, and cases with glaucoma had a similar higher risk to develop DR.

\section{Prevalence and Progression of Diabetic Retinopathy and Macular Edema during Pregnancy in Women with Type I and Type 2 Diabetes}

E.R. Mathiesen', N. Pappot', N.C. Do', J.N. Hajari², H. LundAndersen ${ }^{2}$, P. Holmager', L. Ringholm'

'Center for Pregnant Women with Diabetes, Department of Endocrinology and Metabolism PE7562, Rigshospitalet University of Copenhagen, Denmark ${ }^{2}$ Department of Ophthalmology, Rigshospitalet-Glostrup Hospital, Copenhagen, Denmark

DESIGN. Cohort study

PURPOSE. To evaluate the prevalence and progression of diabetic retinopathy during pregnancy in women with type 1 and type 2 diabetes.
METHODS. In 348 women (223 with type 1 diabetes and 125 with type 2 diabetes) evaluation of retinopathy with retinal photo screening was performed at 12 and 28 weeks and more frequently if indicated.

Severity of diabetic retinopathy was classified in five stages and macular edema in three. Sight-threatening retinopathy was defined as presence of either proliferative diabetic retinopathy or clinically significant macular edema (CSME).

RESULTS. In women with type 1 diabetes $52 \%$ were diagnosed with retinopathy at some point during pregnancy, compared with $14 \%$ in women with type 2 diabetes. Sight-threatening retinopathy was identified in $16 \%$ of women with type 1 diabetes and in $6 \%$ of women with type 2 diabetes.

Women with sight-threatening retinopathy were characterized by retinopathy present at baseline, longer diabetes duration and was mainly identified in women with type 1 diabetes, while women without retinopathy were characterized by shorter diabetes duration and predominance of type 2 diabetes.

Treatment for proliferative diabetic retinopathy during pregnancy was given in five eyes $(0.7 \%)$, belonging to three women who had proliferative diabetic retinopathy at baseline. Treatment for CSME was given in six eyes $(0.9 \%)$ in six different women where visual impairment also was present.

Among 175 women without retinopathy at 12 weeks and with $\mathrm{HbAlc}<53 \mathrm{mmol} / \mathrm{mol}(7.0 \%)$ at baseline, representing half of the total cohort, $165(94 \%)$ remained without retinopathy and none developed sight-threatening retinopathy during pregnancy.

CONCLUSIONS. Retinopathy during pregnancy was present in $52 \%$ of the women with type 1 diabetes and in $14 \%$ of the women with type 2 diabetes. Sight-threatening retinopathy requiring treatment during pregnancy occurred in a minority, and not in women without retinopathy having good glycaemic control in early pregnancy.

In perspective, retinal photo screening in early pregnancy identifies a large group of women at low risk of progression of diabetic retinopathy, while those identified with sight-threatening retinopathy require closer follow-up.

\section{Do Systematic Diabetic Retinopathy Screening Programmes Prevent Severe Sight Impairment?}

C. Styles', N. O'Connell', M. Anderson'

'Department of Ophthalmology, Queen Margaret Hospital, Dunfermline, Fife, UK

DESIGN. Retrospective analysis of annual data.

PURPOSE. Diabetic retinopathy screening aims to prevent severe sight impairment (SSI) through early diagnosis and treatment. Sight threatening complications are also 
related to duration of diabetes, glycaemic control and management of blood pressure. Improvements in the treatment of diabetic eye disease over the last decade, in particular sight threatening maculopathy with anti-vascular endothelial growth factor (VEGF) injections can also prevent sight loss.

We aimed to examine the potential contributions of these factors in sight loss since the introduction of Scotland's systematic national screening programme in 2006.

METHODS. We analysed new SSI certification due to diabetic eye disease in Fife, Scotland between 2000-19. Annual incidence of SSI was calculated from National Records of Scotland population estimates and SCIdiabetes, Scotland's national diabetes register, which also provided data on duration of diabetes, $\mathrm{HbAlc}$ and blood pressure, which were analysed for the period 2006-2019.

RESULTS. The incidence of SSI reduced significantly $(\mathrm{P}<0.001)$ from 59.7 to 16.4 per 100,000 per year, between 2000 and 2019. SSI due to diabetic maculopathy was uncommon, with 3/34 registrations between 2010-19, and $6 / 43$ between $2000-09$.

In $2006,23.1 \%$ of people with diabetes in Fife had retinopathy screening. In 2010 this was $86.6 \%$ and has remained over $80 \%$ since then. In $2006,9.1 \%$ of people had diabetes for less than 1 year and $7.6 \%$ for over 20 years, compared to $3.1 \%$ and $44.5 \%$ in 2019 . In $2006,55 \%$ had an $\mathrm{HbA} 1 \mathrm{c}$ of less than $75 \mathrm{mmol} / \mathrm{mol}$ compared with $68 \%$ in 2019 . In $2006,64.7 \%$ met the blood pressure target compared to $72.1 \%$ in 2019 .

CONCLUSIONS. The significant reduction in severe sight impairment due to diabetes in our region since 2000 suggests that there have been successful interventions over the last 2 decades. Glycaemic and blood pressure control have improved since the introduction of screening. Prevention of sight loss is multifactorial so it is difficult to prove a direct link between implementation of diabetic retinopathy screening and reduction in severe sight impairment. It is important that all these factors are considered together to further reduce preventable sight loss in people with diabetes.

\section{The Levels of Placental Growth Factor and Other Proteins in the Vitreous of Patients Undergoing Diabetic Vitrectomy and the Effect of Bevacizumab as Compared to Aflibercept Pre- Treatment}

\author{
I. Klaassen', D.H.W. Steel' ${ }^{2}$ R.O. Schlingemann' \\ 'Ocular Angiogenesis Group, Amsterdam UMC, The Netherlands \\ ${ }^{2}$ Institute of Genetic Medicine, Newcastle upon Tyne and Sunderland Eye \\ Infirmary, UK
}

DESIGN. Case-control study and experimental study PURPOSE. Proteins are known to interact in networks rather than in isolation, and we sought to investigate the differences in protein levels including placental growth factor (PlGF) and vascular endothelial growth factor (VEGF) in patients with PDR with variable disease severities and signs, including after pretreatment with either bevacizumab/ranibizumab or aflibercept. Additionally, interference of aflibercept with detection of proteins in immunoassays was analysed.

METHODS. We prospectively collected vitreous in a consecutive cohort of patients undergoing vitrectomy for the complications of diabetic retinopathy $(\mathrm{N}=74)$ and macular holes as controls $(\mathrm{N}=52)$, and analysed the samples for a range of growth factors and cytokines in a custom quantitative antibody array and by ELISA. A proportion of patients were pre-treated with either bevacizumab/ranibizumab $(\mathrm{N}=13)$ or aflibercept $(\mathrm{N}=25)$ as part of their pre-operative preparation for surgery and we investigated the effect of these agents on the measured protein levels.

RESULTS. VEGF (98-fold higher in PDR) was significantly lower in the bevacizumab/ranibizumab and aflibercept treated group as compared to the untreated group. PlGF (75-fold higher in PDR) was reduced in both treatment groups, but more in the aflibercept group. In the aflibercept treatment group, concentrations of a number of proteins was decreased, especially that of FLT1 (VEGFR1). Decreasing concentrations of VEGF, PlGF or FLT1 in the presence of increasing amounts of aflibercept were detected by immunoassays. Whereas increased recovery of PlGF was found after extended incubation of PlGF:aflibercept complexes in the immunoassays, the recovery of FLT1 after extended incubation of FLT1:aflibercept complexes was similar to that of standard incubation in the immunoassays.

CONCLUSIONS. In the present study differences in protein levels were observed in the vitreous of patients treated with aflibercept or bevacizumab that may underlie the differences in improvements in patients with PDR. Moreover, here we demonstrate the interference of aflibercept in the detection of VEGF and PIGF and we found strong evidence that aflibercept can also bind FLT1.

\section{The Risk for Developing Vision Threatening Retinopathy after Cataract Surgery in Patients With Diabetes Depends on the Post-Operative Follow-Up Time}

T. Bek', K.Tilma', M.la Cour ${ }^{2}$

'Aarhus University Hospital, Denmark

${ }^{2}$ Rigshospitalet, Denmark

DESIGN. Retrospective cohort study

PURPOSE. To identify parameters that can predict the post-operative risk for progression of retinopathy to a vision threatening stage after cataract surgery. This may 
optimize the timing of cataract surgery in patients with diabetes.

METHODS. Multi-state survival analysis with death as competing risk was used to investigate how year and age of onset of diabetes, gender, body mass index, $\mathrm{HbAlc}$ and blood pressure had affected the risk for developing diabetic macular edema (DME) and proliferative diabetic retinopathy (PDR) among 2,540 right eyes from 2,797 patients with diabetes operated for cataract during 25 years until $1^{\text {st }}$ July 2019.

RESULTS. Cataract surgery had been performed in nearly all patients reaching 90 years of age. The risk of developing both DME and PDR was increased by cataract surgery. The risk was highest during the first post-operative years and was increased by pre-operative variability in $\mathrm{HbAlc}$. The risk after more than 20 years post-operatively was increased by increased cumulative $\mathrm{HbA} 1 \mathrm{c}$ pre-operatively. The other studied risk factors contributed differently to the development of the two complications.

CONCLUSIONS. Decision models for the timing of cataract surgery in patients with diabetes should consider that the risk for developing vision threatening retinopathy depends on follow-up time. Differences in the risk profiles for developing DME and PDR after cataract surgery supports that the two complications should be regarded as separate late complications.

\section{Are there Still Indications for Macular Laser in Diabetic Macular Oedema?}

\author{
A. Dale', C.F.E. Norridge', P.H.J. Donachie', Q. Mohamed', \\ P.H. Scanlon' \\ 'Gloucestershire Retinal Research Group, Gloucestershire Hospitals NHS \\ Foundation Trust, Gloucestershire, UK
}

DESIGN. Inclusion criteria were people with diabetes commencing treatment with macular laser recorded on the ophthalmology electronic medical record system with an indication for laser treatment of Clinically Significant Macular Oedema (CSMO), with baseline Optical Coherence Tomography (OCT) measurements from the same machine, within 3 months before and 1-12 months after treatment. Eyes were excluded if their central subfield retinal thickness (CRT) was $\geqslant 400$ microns, they had ocular co-pathologies or surgery in the previous 6 months or OCT measurements on different machines.

PURPOSE. To audit outcomes of macular laser therapy in patients with diabetes and predominantly non-centre involving clinically significant macular oedema in a UK centre.

METHODS. A retrospective analysis of people receiving their first macular laser treatment between 01/01/2010 and 31/12/2019 and OCT measurements, within 3 months before and 1-12 months after treatment.
RESULTS. Eligible for analysis were 316 eyes from 275 patients with 175 (63.6\%) male and $100(36.4 \%)$ female, 29 (10.6\%) T1DM and 246 (89.4\%) T2DM. The median age was 63.1 years (range; 28.2 - 90.9 years). Altogether, 253 eyes had Zeiss Cirrus OCT measurements, with mean baseline CRT 303.6 microns, mean follow up CRT 305.1 microns and mean difference +1.5 microns $(95 \% \mathrm{CI}$ : -4.750 to 7.659 microns). In only two macular regions did $<50 \%$ of eyes have a reduction in thickness. From 63 eyes with Heidelberg OCT measurements, the mean baseline CRT was 302.4 microns, the mean follow up CRT was 309.4 microns and the mean difference was +7.0 microns ( $95 \%$ CI: -4.733 to 18.764 microns). In all macular regions $>50 \%$ of eyes had a reduction in thickness. After VEGF inhibitor injections became available in the UK in 2013, only $3(5.4 \%)$ eyes with Heidelberg and 10 (10.5\%) eyes with Zeiss OCT measurements received injections within 1 year of laser. The median baseline and follow up VA were both 0.20 LogMAR, and the median change in VA was 0.00 LogMAR.

CONCLUSIONS. These results suggest that there is still a place for macular laser treatment in predominantly noncentre involving diabetic macular oedema.

\section{Effect of Calcium Dobesilate in Patients With Subclinical Diabetic Macular Edema: The Cadodiame Study}

O. Simó-Servat' ${ }^{\prime}$ A.S. Simó-Servat ${ }^{2}$, X.V. Valldeperas ${ }^{3}$, C.H. Hernández', L.L. Lehr", D.Z. Zingg ${ }^{4}$, R.S. Simó'

'Department of Endocrinology, Hospital Vall d'Hebron, Barcelona, Spain ${ }^{2}$ Department of Endocrinology, Hospital Mutua de Terrassa, Terrassa, Spain ${ }^{3}$ Department of Ophthalmology, Hospital Germans Trias i Pujol, Badalona, Spain

${ }^{4}$ OM-Pharma, Geneve, Switzerland

DESIGN. Randomized placebo-controlled clinical trial PURPOSE. Several randomized placebo-controlled clinical trials have shown that calcium dobesilate $(\mathrm{CaD})$ arrest the progression of early DR, but more supportive evidence is needed. Patients with subclinical DME represent a good target population to evaluate therapeutic strategies for preventing CSME. On this basis, we aimed to test the effect of $\mathrm{CaD}$ in early stages of $\mathrm{DR}$, including the progression from subclinical DME to CSME.

METHODS. In this randomized, multicenter placebocontrolled study of parallel groups, 60 eligible patients with type 2 diabetes with subclinical DME were randomized 1:1 to receive $\mathrm{CaD}$ (Doxium ${ }^{\circledR}, 1000 \mathrm{mg}$ twice per day) or placebo for 1 year. The primary endpoint was to determine whether $\mathrm{CaD}$ is able to prevent the increase of retinal thickness (RT). The secondary endpoints were to assess whether $\mathrm{CaD}$ is able to arrest: 1) the progression from subclinical to CSME; 2) the progression of the 
ETDRS level ( $\geqslant 1$ step); 3 ) the progression of neurodegenerative changes, 4) the reduction of visual acuity, 5) the progression of foveal avascular zone.

RESULTS. A total of 50 patients completed the 1-year follow-up. RT decreased by a mean of $2.5 \mu \mathrm{m}$ (SD14) in patients treated with $\mathrm{CaD}$, whereas in patients treated with placebo RT increased by $3.8 \mu \mathrm{m}$ (SD24). However, this difference does not reach statistical significance $(\mathrm{p}=$ 0.26). Regarding secondary endpoints the only difference was the effect of $\mathrm{CaD}$ on the progression of ETDRS severity level. We found an improvement of ETDRS severity level in 9 of patients in the $\mathrm{CaD}$ in comparison with 3 in the placebo group ( $37.5 \%$ vs. $12 \% ; \mathrm{p}=0.05)$. In the logistic regression analyses adjusting for age, hypertension and the mean of $\mathrm{HbAlC}$, treatment with $\mathrm{CaD}$ was independently associated with an improvement of ETDRS severity level at the end of 1-year follow-up ( $\mathrm{P}=0.03)$.

CONCLUSIONS. In our study, the treatment with $\mathrm{CaD}$ did not have any significant impact on the progression of RT, at least not at one year of follow-up. However, it was a useful treatment for early microvascular impairment in the setting of DR as shown by the improvement of ETDRS level.

\section{Factors Associated with the Response to Fluocinolone Acetonide $0.19 \mathrm{mg}$ in Diabetic Macular Edema Evaluated as the Area-Under- The-Curve}

M.V. Cicinelli', L. Capone', C. Di Biase', R. Lattanzio', F. Bandello'

'Department of Ophthalmology, IRCCS Ospedale San Raffaele, Milano, Italy

DESIGN. Retrospective clinical study

PURPOSE. To investigate the impact of different baseline demographic, clinical, and optical coherence tomography (OCT) factors on the functional and morphologic response to fluocinolone acetonide (FAc) $0.19 \mathrm{mg}$ implant in patients with diabetic macular edema (DME), evaluated as the area-under-the-curve (AUC).

METHODS. Retrospective study of pseudophakic eyes with DME undergoing FAc implant. The AUC of the bestcorrected visual acuity (BCVA expressed as LogMAR) and the central macular thickness (CMT) changes up to 36 months were calculated with the trapezoidal rule. The AUC values were divided by the amount of available follow-up (expressed in months) of each eye, to correct for the heterogeneous follow-up. Demographic and clinical data and OCT features at the time of FAc administration were collected, and their associations with BCVA and CMT AUC were investigated with linear mixed models.

RESULTS. Eighty-one eyes of 63 patients with a minimum 12-month follow-up were enrolled; median available follow-up was 26 months. The mean AUCBCVA after FAc injection was $0.24 \pm 0.17 \mathrm{LogMAR} /$ month. Lower AUCBCVA (better vision during follow-up) was associated with higher baseline BCVA $(\mathrm{p}<0.001)$, lower AUCCMT after FAc administration ( $\mathrm{p}<0.001)$, type 2 diabetes $(\mathrm{p}=0.04)$, intact subfoveal ELM/EZ layer $(\mathrm{p}=0.01)$.

CMT significantly decreased after FAc $(p<0.001)$. The mean AUCCMT was $179.6 \pm 54.3 \mu \mathrm{m} /$ month. Lower AUCCMT (thinner macula during the follow-up) was associated a history of anti-VEGF injections administered before FAc $(p<0.001)$. Eyes with higher CMT at baseline $(p<0.001)$ and those with tractional DME $(p=0.01)$ had higher AUCCMT during the follow-up. The need of additional treatments after FAc was also associated with higher AUCCMT for 36 months $(\mathrm{p}=0.001$ )

CONCLUSIONS. Baseline visual acuity, retinal thickness, and photoreceptors' integrity were associated with better functional response to FAc implant over time; severe macular edema and tractional DME had a worse response to intravitreal sustained-release corticosteroids. In our cohort of eyes with long-standing DME, the duration of the disease was not associated with treatment outcomes. These findings might guide clinicians in a more informed decisional algorithm in treating DME.

\section{Macular Function and Structure in Early Diabetic Retinopathy}

J.Y. Ku', 2, S.P. Harding', 2, G. Czanner', 3, W. Zhu', P.C. Knox'

'Department of Eye and Vision Science, University of Liverpool, Member of Liverpool Health Partners, Liverpool, UK

${ }^{2}$ St. Pauls Eye Unit, Liverpool University Hospitals, Member of Liverpool Health Partners, Liverpool, UK

${ }^{3}$ Department of Biostatistics, John Moores University, Member of Liverpool Health Partners, Liverpool, UK

DESIGN. Single centre, prospective cross-sectional controlled observational study

PURPOSE. The effect of diabetes on retinal biology in early disease is poorly understood. We investigated functional and structural alterations in the macula in people with diabetes (PWD) with early diabetic retinopathy.

METHODS. PWD referred from the Liverpool Diabetic Eye Screening Programme (LDESP) to hospital clinics as being at risk of diabetic macular oedema (DMO) and agematched healthy controls (HC) were recruited. Macular function was measured using handheld radial shape discrimination (hRSD), distance and near visual acuity (VA). Macular structure was measured using optical coherence tomography (OCT; Spectralis, Heidelberg Eye Explorer 1.10.0.0, version 6.8a). Retinal layer segmentation was performed using Heidelberg auto-segmentation software with manual adjustment as needed. Full and individual retinal layer thickness across all Early Treatment Diabetic 
Retinopathy Study (ETDRS) subfields were measured. One eye from each participant was randomly selected for analysis.

RESULTS. 292 PWD (mean \pm SD age $54 \pm 14$ yrs, 175 males) cases and $50 \mathrm{HC}(55 \pm 14 \mathrm{yrs}, 26$ males $)$ were recruited. Compared to $\mathrm{HC}$, hRSD performance and distance VA were progressively worse in PWD with no or minimal diabetic retinopathy (DR), (hRSD logMAR: HC $-0.77 \pm 0.11$, no DR $-0.68 \pm 0.18$, minimal DR $-0.61 \pm 0.25$, ANOVA $p<0.001$; distance VA $\log$ MAR: HC $-0.08 \pm 0.12$, no DR $0.03 \pm 0.15$, minimal DR $0.06 \pm 0.16$, ANOVA $\mathrm{p}<0.001)$. There was also a reduction in full retinal thickness across most subfields. Compared to $\mathrm{HC}$, retinal nerve fibre layer thickness was reduced in outer subfields (OS) in PWD with no DR $(4.6 \mu \mathrm{m}, 12.3 \%$ difference, $\mathrm{p}<0.001)$ or minimal DR $(2.7 \mu \mathrm{m}, 7.2 \%, \mathrm{p}=0.015)$. Compared to HC, ganglion cell layer thickness was reduced in the in inner subfields (IS) in PWD with no DR $(4.1 \mu \mathrm{m}, 8 \%, \mathrm{p}=0.003)$ and in both IS and OS in PWD with minimal DR $(5.1 \mu \mathrm{m}$, $9.9 \%, \mathrm{p}<0.001 ; 1.9 \mu \mathrm{m}, 5.5 \%, \mathrm{p}=0.015$, respectively).

CONCLUSIONS. Functional and structural changes are detectable in the early pathogenesis of DR, consistent with inner neuroretinal thinning developing before microvascular abnormalities. Our findings add further support to the concept of "pre-clinical retinopathy". Future studies to explore OCT thickness measurements as surrogate markers for DR neurodegeneration will be useful.

\section{Early Worsening of Retinopathy in People with Diabetes after Rapid Improvement in Glycaemic Control: A Systematic Review}

H. Akil ${ }^{1,2}$, J.B. Burgess ${ }^{3}$, S.N. Nevitt ${ }^{4}$, S.H. Harding ${ }^{1,2}$, U.A. Alam $^{3,5}$, P.B. Burgess ${ }^{1,2}$

'Department of Eye and Vision Science, Institute of Ageing and Chronic Diseases, University of Liverpool, Liverpool, UK

${ }^{2}$ St. Paul's Eye Unit, Liverpool University Hospitals NHS Trust, Liverpool, UK ${ }^{3}$ Diabetes \& Endocrinology Research, Institute of Cardiovascular and Metabolic Medicine and The Pain Research Institute, University of Liverpool and Liverpool University NHS Hospital Trust, Liverpool, UK

${ }^{4}$ Department of Health Data Science, University of Liverpool, Liverpool, UK ${ }^{5}$ Division of Endocrinology, Diabetes and Gastroenterology, University of Manchester, Manchester, UK

DESIGN. Systematic review

PURPOSE. To systematically review the epidemiology of early worsening of diabetic retinopathy (EWDR) after substantial improvements in glycaemic control and estimate review the epidemiology and characteristics including risk factors.

METHODS. An electronic literature search was performed according to PRISMA guidelines using MEDLINE, EMBASE, PubMed, Web of Science, Scopus and Cochrane databases and manual reference for the articles published until 2020. Published full-text English language articles that report data on diabetic retinopathy in people with diabetes experiencing a rapid, substantial decrease in $\mathrm{HbA} 1 \mathrm{c}$ after going through intensive therapy were included. All articles were screened, data extracted and methodological quality evaluated by two independent reviewers using a priori criteria.

RESULTS. 346 articles were identified after the removal of duplicates. Data were extracted from 19 full text articles with a total of 15,588 participants. Included studies varied considerably in terms of patient selection and method of assessing the eye and retinopathy classification. EWDR was reported to occur in a wide range of prevalence's 3.3 to $47 \%$ (IQR: 12 ) of participants within 3-84 months after intensification of glycaemic control. Risk factors for EWDR included long-duration of diabetes, long term uncontrolled hyperglycemia and baseline retinopathy severity in both type 1 and type 2 diabetes. The occurrence of EWDR and progression of retinopathy were found to have an association with the amplitude of $\mathrm{HbA} 1 \mathrm{c}$ reduction.

CONCLUSIONS. EWDR has been described in a proportion of people with intensification of glycaemic control. However, the prevalence remains unclear due to methodological differences in the identified studies. Future interventional studies should report retinopathy and visual outcomes using standardised protocols.

\section{Mfap4 Immunotherapy-Mediated Inhibition of Retinal Neovascularization and Vascular Leakage}

A. Schlosser 1,2, B. Pilecki', J. Grauslund ${ }^{4}$, T.L. Andersen ${ }^{3}$, S. Heegaard $^{6}$, D.O. Bates' ${ }^{2}$ G.L. Sorensen'

'Department of Molecular Medicine, University of Southern Denmark, 5000 Odense, Denmark

${ }^{2}$ Cancer Biology, Division of Cancer and Stem Cells, School of Medicine, University of Nottingham, Nottingham UK

${ }^{3}$ Clinical Cell Biology, Department of Pathology, Odense University Hospital, 5000 Odense, Denmark

${ }^{4}$ Department of Ophthalmology, Odense University Hospital, 5000 Odense, Denmark

${ }^{5}$ Department of Pathology, Rigshospitalet, University of Copenhagen, 2100

Copenhagen, Denmark

${ }^{6}$ Department of Ophthalmology, Rigshospitalet-Glostrup, University of Copenhagen, Copenhagen, 2600 Glostrup, Denmark

DESIGN. Neovascular age-related macular degeneration (nAMD) and diabetic macular edema (DME) are leading causes of visual disability in the elderly and workingage populations, respectively. Integrins alphaVbeta3 and alphaVbeta5 are implicated in main pathologic disease hallmarks like neovascularization and vascular leakage. Microfibrillar-associated protein 4 (MFAP4) is integrin alphaVbeta3/5 ligand expressed in the vascular extracellular matrix. We envision that therapeutic blocking of MFAP4 can benefit nAMD and DME and we therefore produced MFAP4 blocking antibodies 'hAS036' and tested 
the beneficial effect using preclinical in vitro and in vivo disease models.

PURPOSE. The purpose was to obtain preclinical proofof-concept of efficacy.

METHODS. Immunohistochemistry and in situ hybridization was used to detect MFAP4 expression in the human eye. hAS0326 was produced by immunization of MFAP4deficient mice with human MFAP4 and consecutive hybridoma technique and finally humanized. hAS0326 efficacy was tested using retinal endothelial cell adhesion, proliferation and migration assays and mouse model of laser-induced choroidal neovascularization (CNV), rat model of streptozotocin (STZ)-induced retinopathy, and African green monkey model of DL-2-aminoadipic acid (DLAAA)-induced chronic retinopathy.

RESULTS. MFAP4 was expressed in retina and choroid of the human eye. hAS0326 blocked integrin-dependent endothelial cell adhesion, proliferation and migration in vitro and had efficacy at par with anti-VEGF in models of mouse laser-induced CNV, rat streptozotocin-induced retinopathy and in a chronic non-human primate model DLAAA-induced model of retinopathy. In the latter model, one intravitreal dose of hAS0326 exerted $>3$ months duration of efficacy in reduction of vascular leakage.

CONCLUSIONS. These data suggest that hAS0326 is a promising stand-alone therapy against a novel target for the treatment of vision-threatening retinal vascular eye diseases such as nAMD and DME.

\section{Diabetic Retinopathy and Skin Tissue Advanced Glycation End Products are Biomarkers of Vascular Events in Patients with Type 2 Diabetes: Results of the "Precised" Study}

A. Planas ${ }^{1,2}$, O. Simó-Servat
C. Hernández ${ }^{1,2}$, I. Merreira-González ${ }^{3}$, R. Simón ${ }^{2}, 2$
'Diabetes and Metabolism Research Unit Vall d'Hebron Research Institute,
Barcelona, Spain
${ }^{2}$ Department of Endocrinology and Nutrition, Hospital de Vall d'Hebron,
Barcelona, Spain
${ }^{3}$ Cardiology Research Unit. Vall d'Hebron Research Institute, Barcelona, Spain

DESIGN. Prospective case-control study.

PURPOSE. The early identification of those patients with diabetes who are at high risk of developing cardiovascular events (CVE) remains a challenge. The aim of this study is to evaluate whether the presence of diabetic retinopathy (DR) and accumulation of advanced glycation end products (AGEs) in subcutaneous tissue can help to identify those patients with type 2 diabetes (PwT2D) at high risk of (CVE).

METHODS. It was a prospective case-control study comprising 200 PwT2D with no history of CVE and 60 non-diabetic controls, matched by age and sex (PRECISED study:
ClinicalTrial. Gov NCT02248311). The inclusion period began on September 2014 and finished on June 2017. We collected basal features of the subjects, classical cardiovascular risk factors (i.e. age, sex, hypertension, dyslipidemia and coronary artery calcium score [CACs]), presence and degree of DR, and the accumulation of advanced glycation end products in subcutaneous tissue using the AGE readerTM device (DiagnOptics Technologies). We followed these subjects until December 2020, collecting any coronary, cerebrovascular or peripheral arterial event. We analyzed the differences between PwT2D who presented a CVE compared to those who had not.

RESULTS. After a follow up of $4.35 \pm 1.43$ years, a total of $24 \mathrm{CVE}$ were registered. The number of CVE was higher in PwT2D than in the control group $(12.3 \%$ vs. $1.75 \%, \mathrm{p}=0.035)$. PwT2D with CVE presented a higher prevalence of DR (47.8\% vs. $24.4 \%$; $p=0.018)$ and AGEs in subcutaneous tissue $(63.15 \%$ vs $26.71 \%$ of values in the higher tertile, $\mathrm{p}=0.001$ ). We observed that sex, gender, $\mathrm{DR}$, CACs and AGEs were independent predictors of CVE in the Cox's regression analysis.

CONCLUSIONS. DR and high levels of AGEs in subcutaneous tissue are independent predictors of CVE and could help to identify those PwT2D in whom a more tight control of risk factors should be recommended.

\section{Octa Metrics Monitor Severity Progression of Diabetic Retinopathy}

J. Cunha-Vaz', 2, I.P. Marques', 2, T. Santos', M.H. Madeira', 2 , A.R. Santos ${ }^{1,4}$, C. Lobo', 2, 5 , M. Durbin ${ }^{3}$

'AIBILI - Association for Innovation and Biomedical Research on Light and Image, Coimbra, Portugal

${ }^{2}$ University of Coimbra, Coimbra Institute for Clinical and Biomedical Research (iCBR), Faculty of Medicine, Coimbra, Portugal

${ }^{3}$ Research and Development, Carl Zeiss Meditec, Dublin, California, USA ${ }^{4}$ Department of Orthoptics, School of Health, Polytechnic of Porto, Porto, Portugal

${ }^{5}$ Department of Ophthalmology, Centro Hospitalar e Universitário de Coimbra (CHUC), Coimbra, Portugal

DESIGN. 3-year longitudinal study

PURPOSE. To examine retinal vessel closure metrics and neurodegenerative changes occurring in the initial stages of nonproliferative diabetic retinopathy (NPDR) and severity progression in a three-year period.

METHODS. Three-year prospective longitudinal observational cohort of eyes/patients with type 2 diabetes (T2D) using spectral domain-optical coherence tomography (SD-OCT) and OCT-Angiography (OCTA). Eyes were examined four times with one-year intervals. OCTA vessel density maps of the retina were used to quantify vessel closure. Thickness of the ganglion cell inner plexiform layer (GCL+IPL) was examined to identify retinal neurodegenerative changes. Diabetic retinopathy ETDRS 
classification was performed using the seven-field ETDRS protocol.

RESULTS. A total of 78 eyes/patients, aged 52 to 80 years, with T2D and ETDRS grades from 10 to 47 were followed for 3 years with annual examinations. A progressive increase in retinal vessel closure demonstrated by higher decreases in vessel density (VD) correlated with retinopathy worsening identified by step-changes in ETDRS severity scale $(p<0.001)$. This decrease in VD varied between different individuals. No apparent correlation was found between neurodegenerative changes and retinopathy progression.

CONCLUSIONS. Retinal vessel closure in NPDR correlates with DR severity progression. Our findings provide evidence to support that OCTA metrics of vessel closure may be used as a surrogate for DR severity progression.

\section{Ganglion Cell-Inner Plexiform Layer Alteration in the Initial Stages of Diabetic Retinopathy in Type 2 Diabetic Patients: A 5-Year Longitudinal Study}

M.H. Madeira' ${ }^{1.2 .3}$, D. Tavares', I.P. Marques ${ }^{1,2,3}$, A.R. Santos ${ }^{1,2,3,4}$, T. Santos', C. Lobo' ${ }^{1,2,3,4,5}$, J. Cunha-Vaz ${ }^{1,2,3}$

'AIBILI, Association for Innovation and Biomedical Research on Light and Image, Coimbra, Portugal

${ }^{2}$ Coimbra Institute for Clinical and Biomedical Research (iCBR), Faculty of Medicine, University of Coimbra, Coimbra, Portugal

${ }^{3}$ Center for Innovative Biomedicine and Biotechnology (CIBB), University of Coimbra, Coimbra, Portugal

${ }^{4}$ Department of Orthoptics, School of Health, Polytechnic of Porto, Porto, Portugal

${ }^{5}$ Department of Ophthalmology, Centro Hospitalar e Universitário de Coimbra (CHUC) Coimbra, Portugal

DESIGN. 5-year prospective, longitudinal study of individuals with type 2 diabetes (T2D) with mild nonproliferative diabetic retinopathy (NPDR, ETDRS level 20 or 35). PURPOSE. Diabetic Retinopathy (DR) has always been considered a microvascular disease, but it has been suggested that neurodegeneration plays a key role in its complex pathology. We evaluated the progression of DR neurodegeneration, considering ganglion cell + inner plexiform layers (GCL + IPL) thinning, and its correlation with microvascular disease, visual acuity, ETDRS grade and severity progression.

METHODS. We included 212 T2D individuals of which 145 completed the 5-year follow-up, with ophthalmological examinations at baseline, 6-months and annually (one eye per patient). GCL+IPL average thickness was evaluated by optical coherence tomography (OCT). ETDRS level and severity progression were assessed by grading of 7-fields color fundus photography. Microaneurysm turnover (MAT) was evaluated using the RetMarkerDR. Visual acuity (BCVA) was measured using Snellen chart and converted $\log$ MAR VA. A population of 58 healthy individuals was used as baseline reference. Statistically differences between continuous variables were assessed by Mann-Whitney test. Linear mixed effects models with patient random effect patient were used to analyze GCL+IPL progression over-time and the effect of variables of interest.

RESULTS. Baseline GCL+IPL thickness $(80.06 \pm 7.71$ $\mu \mathrm{m})$ in T2D individuals was significantly reduced when compared to controls $(82.67 \pm 5.46 \mu \mathrm{m}, \mathrm{p}=0.02)$, indicating the presence of neurodegenerative changes since the initial stages of retinopathy. Through the 5 -year follow up there was progressive GCL + IPL thinning $(p<0.001)$, occurring independently of the baseline ETDRS levels (20 or 35) and microvascular changes (evaluated by MAT). Although progressive neurodegeneration was identified in 5-years of follow-up, it did not show an association with worsening of DR severity (ETDRS step changes). Additionally, GCL+IPL thinning (difference V6-V1), was shown to be associated with BCVA worsening $(\mathrm{p}=0.021)$.

CONCLUSIONS. Neurodegeneration, represented by GCL+IPL thinning, is present since the initial stages of NPDR and progresses significantly over a 5 -year period, independently of the microvascular alterations (identified by MAT), ETDRS grade and DR severity progression. Neurodegenerative changes are associated with visual acuity loss during the 5-year period of follow-up.

\section{Microaneurysm Turnover in Eyes with Mild Nonproliferative Diabetic Retinopathy is Associated with Progression and Development of Sight-Threatening Complications: A 5-Year Longitudinal Study}

A.R. Santos 1,2, I.P. Marques',3, C. Lobo',3,4, M.H. Mendes',3, D. Tavares', J. Figueira ${ }^{1,3,4}$, J. Cunha-Vaz ${ }^{1,3}$

'AIBILI - Association for Innovation and Biomedical Research on Light and Image, Coimbra, Portugal

${ }^{2}$ Department of Orthoptics, School of Health, Polytechnic of Porto, Porto, Portugal

${ }^{3}$ University of Coimbra, Coimbra Institute for Clinical and Biomedical Research (iCBR), Faculty of Medicine, Coimbra, Portugal

${ }^{4}$ Department of Ophthalmology, Centro Hospitalar e Universitário de Coimbra (CHUC), Portugal

DESIGN. A 5-year prospective, longitudinal study PURPOSE. To evaluate microaneurysm turnover (MAT) as a predictive biomarker for retinopathy progression and development of sight-threatening complications, diabetic macular edema (DME) or proliferative retinopathy (PDR), in patients with type 2 diabetes and mild nonproliferative retinopathy (NPDR) in a 5-year follow-up.

METHODS. 212 individuals with type 2 diabetes (T2D; ETDRS grades 20 and 35) were evaluated annually in a 5 -year prospective, longitudinal study, by color fundus photography and optical coherence tomography. Endpoints 
were diabetic macular edema (DME) or proliferative retinopathy (PDR). MAT analysis included determination of MA formation and disappearance rates, automatically assessed using the RetMarkerDR $®$. Retinopathy severity progression was evaluated using step increases in ETDRS severity levels.

RESULTS. Of the 212 individuals, 172 completed the 5 -year follow-up study or developed an endpoint $(\mathrm{n}=27)$. MAT calculated at 1 year showed a significant association with the development of any endpoint $(\mathrm{p}=0.018)$, in particularly MA disappearance rate $(\mathrm{p}=0.007)$. MAT showed also significant association with ETDRS severity worsening in the 5-year period $(\mathrm{p}=0.035)$. There was a clear increase in the MA disappearance rate being observed in the last year of the study $(1.50 \pm 1.93)$ compared with the first-year of follow-up $(1.05 \pm 1.99 ; \mathrm{p}=0.003)$.

CONCLUSIONS. MAT is an indicator of the development of DME and/or PDR as well as of DR severity progression in $\mathrm{T} 2 \mathrm{D}$ individuals with mild retinopathy.

\section{Three-Year OCT-Angiography Evaluation in Patients with Diabetes Mellitus Type I and 2 Without Clinical Signs of Diabetic Retinopathy and with Good Glycemic Control}

\author{
S. Vujosevic ${ }^{2}$, C. Toma 2 , E. Torti ${ }^{3}$, P. Nucci ${ }^{4}$, S. De Cillà2,5 \\ 'Eye Clinic, IRCCS MultiMedica, Milan, Italy \\ ${ }^{2}$ Eye Clinic, University Hospital Maggiore della Carità, Novara, Italy \\ ${ }^{3}$ Department of Electrical, Computer and Biomedical Engineering, University \\ of Pavia, Italy \\ ${ }^{4}$ Department of Clinical Sciences and Community Health, University of Milan, \\ Milan, Italy \\ ${ }^{5}$ Department of Health Sciences, University of East Piedmont "A Avogadro", \\ Novara, Italy
}

DESIGN. 3-year prospective, longitudinal, case-control comparative study.

PURPOSE. To evaluate and compare microvascular and neuronal changes in the macula in patients with type 1 and 2 diabetes mellitus (DM1/DM2) and no clinical signs of diabetic retinopathy (DR) and healthy controls using optical coherence tomography (OCT)/OCT-angiography (OCT-A).

METHODS. Twenty eyes with DM1, 48 with DM2 and 24 controls underwent a complete eye examination and swept source OCT/OCT-A at baseline and after 3 years. Thickness of retinal nerve fiber layer(NFL), ganglion cell layer $(\mathrm{GCL}+)$ and $\mathrm{NFL}+\mathrm{GCL}+(\mathrm{GCL}++)$ was evaluated on OCT. On OCT-A: FAZ area, perimeter and circularity index; perfusion and vessel density(PD/VD) and fractal dimension(FD) at the superficial and deep capillary plexus(SCP/DCP); choriocapillaris flow voids(CC-FV). Analyses were performed using ImageJ software (version 1.5; http://imagej.nih.gov/ij/) and MATLAB (version 2017b; Mathworks, Niatick, MA).
RESULTS. All patients maintained a good glycemic control throughout the study (mean HbA1c $7.4 \pm 0.8 \%$ at baseline, $7.1 \pm 0.9 \%$ after 3 years in DM1; $7.2 \pm 0.8 \%$ at baseline, $6.8 \pm 1.2 \%$ after 3 years in DM2).No eye developed DR during the follow-up. At baseline subfoveal GCL +/GCL + + were significantly higher in DM1vsDM2 $(\mathrm{p}<0.01)$; SCP-PD was reduced in DM1 vs controls $(\mathrm{p}=0.05)$; FD was reduced in both DM1 and DM2 vs controls in both plexuses $(\mathrm{p}<0.001)$; CC-FV were increased in $\mathrm{DM} 2$ vs DM1 ( $\mathrm{p}=0.02)$ and controls $(\mathrm{p}=0.002)$; FAZ metrics were significantly altered in both DM1 and DM2 vs controls in both plexuses $(p \leqslant 0.03)$. After 3 years: SCP-PD increased $(\mathrm{p}=0.03)$, DCP-PD and CC-FV decreased in $\mathrm{DM} 2(\mathrm{p}=0.007$ and $\mathrm{p}=0.004$ respectively); FAZ area and perimeter at the DCP significantly increased in DM2 $(\mathrm{p}<0.001)$ compared to DM1 and controls.

CONCLUSIONS. During the 3 -year follow-up no patients developed clinically detectable DR in a cohort of DM type $1 \& 2$ patients with good glycemic control. The increase in perfusion at the SCP and CC (secondary to vasodilation) may be due to a compensatory effect to the reduced perfusion at the DCP in the earliest phases of diabetic retinal disease. Longer and larger longitudinal studies are needed to confirm these preliminary data.

\section{Development of an Artificial Intelligence-based Diabetic Retinopathy Screening Tool for Optos Ultra-Widefield Imaging}

\author{
T. Peto', L.P. Aiello², S. Sadda ${ }^{3}$, Rinaldo Study Group \\ 'Centre for Public Health, Queen's University Belfast, UK \\ ${ }^{2}$ Beetham Eye Institute, Joslin Diabetes Center, Harvard Medical School, USA \\ ${ }^{3}$ Doheny Eye Institute, Ronald Reagan UCLA Medical Centre, USA
}

DESIGN: Retrospective, multicentre study.

PURPOSE: OptosAI is intended for the screening of Diabetic Retinopathy (DR) in individuals with diabetes using a machine learning algorithm to analyse ultra-widefield imaging (UWF). This study presents the method for collecting and grading data to support the development of the algorithm.

METHODS: Altogether, 67,200 UWF colour images from patient with diabetes were used in algorithm training. Of these, 30,836 images were collected from diabetic eye screening programs from the United Kingdom and United States with assigned ICDR grades. Images were anonymized, made DICOM compliant and were double graded at eight different grading centres (CARF, DIRRL (2), BEI, DIRC, EyePacs, Apollo, BIRC) by 125 certified graders using the ETDRS extension of the Modified Airlie House Classification of Diabetic Retinopathy following the JVN Clinical Trial Ultrawide Field Grading Manual v1.0. The grading system used traditional ETDRS 7-SF field definition as well as extended fields 3-7 to evaluate 
the periphery. Grading resulted in 179 individual fields of data for each image. Discrepancies were adjudicated by consultant ophthalmologists. A further 36,364 UWF colour images from ophthalmology clinics in Canada, France, India, United Kingdom and United States with DR severity graded using a redacted grading protocol based on the ICDR classification. From the first 20,000 images of 125 graders a randomly selected intra-grader assessment to check the quality of the grading was completed. $33 \%$ of graders with over 100 grades were chosen with $5 \%$ of total images selected. Intra-grader assessment was complete with a defined protocol with an acceptance criterion of $80 \%$ agreement within 1 level.

RESULTS: 332 grades from 10 graders were accessed with an acceptance level $82-100 \%$. Graders exhibited a learning curve with a temporal trend in early cases.

CONCLUSION: OptosAI was trained with the highest quality graded images to support. High quality data, grading and adjudication will support development of an algorithm with the potential to contribute to safe and timely decision so patients are on the most appropriate pathway.

\section{Trustworthy Al: Closing the Gap between Development and Integration of $\mathrm{Al}$ in Ophthalmology}

C. González-Gonzalo',2, F. Verbraak³, R.O. Schlingemann ${ }^{3}$, C.C.W. Klaver ${ }^{4,5,6,7}$, A.Y. Lee ${ }^{8}$, A. Tufail ${ }^{9,10}$, C.I. Sánchez ${ }^{1,11}$

'Eye Lab, qurAl Group, Informatics Institute, University of Amsterdam, Amsterdam, The Netherlands

${ }^{2}$ Diagnostic Image Analysis Group, Department of Radiology and Nuclear Medicine, Radboud University Medical Center, Nijmegen, The Netherlands

${ }^{3}$ Department of Ophthalmology, Amsterdam University Medical Center, Amsterdam, The Netherlands

${ }^{4}$ Department of Ophthalmology, Erasmus Medical Center, Rotterdam, The Netherlands

${ }^{5}$ Department of Epidemiology, Erasmus Medical Center, Rotterdam, The Netherlands

${ }^{6}$ Department of Ophthalmology, Radboud University Medical Center, Nijmegen, The Netherlands

${ }^{7}$ Institute of Molecular and Clinical Ophthalmology, Basel, Switzerland

${ }^{8}$ Department of Ophthalmology, School of Medicine, University of

Washington, Seattle, WA, USA

${ }^{9}$ Moorfields Eye Hospital NHS Foundation Trust, London, UK

${ }^{10}$ Institute of Ophthalmology, University College London, London, UK

"Department of Biomedical Engineering and Physics, Amsterdam University

Medical Center, Amsterdam, The Netherlands

DESIGN. Descriptive study.

PURPOSE. To identify the main aspects that currently complicate the integration of artificial intelligence (AI) in ophthalmic settings.

METHODS. Based on an extensive review of state-ofthe-art literature of AI applied to ophthalmology plus interviews with multidisciplinary, international experts, we identified the most relevant aspects to consider during AI design to generate trustworthy (i.e., transparent, robust, and sustainable) AI systems and, consequently, facilitate a subsequent successful integration in real-world ophthalmic settings.

RESULTS. Several essential aspects to consider were identified:

1. The reliability of the human annotations that are used for establishing the reference standard an AI system learns from, or for setting robust observer studies that allow for fair human-AI performance comparison.

2. The ability of an AI system to generalize across populations, ophthalmic settings, and data acquisition protocols in order to avoid the negative consequences of algorithmic bias and lack of domain adaptation.

3. The integration of multimodal data for AI development to consider multiple contexts when available (phenotyping, genotyping, systemic variables, patient medical history).

4. The importance of providing interpretable AI-based predictions to open the "black box" and increase trust and clinical usability.

5. A plan to monitor the impact of AI on the clinical workflow, i.e., the adaptation of healthcare providers and patients to the new technology, human-AI interaction, cost-benefit analyses. . .

6. The necessity to update current regulations to accelerate and control AI integration and all related aspects, such as patient privacy, systems' updates, and liability.

CONCLUSIONS. It is important that healthcare providers in ophthalmology consider these aspects and their consequences when thinking of $\mathrm{AI}$ in practice. It is key that all involved stakeholders collaborate and interact from the beginning of the AI design process to ensure a good alignment with real-world clinical needs and settings. This way, it will be possible to generate trustworthy AI solutions and close the gap between development and deployment, so that the AI benefits currently shown on paper reach the final users.

\section{Accuracy of a Diabetic Retinopathy Grading Algorithm used with a Hand-Held Fundus Camera in Southern Malawi}

\author{
J. Aslan ${ }^{1,2}$, P. Kayange 1,2, P. Soliz ${ }^{4}$, S. Mdala², T. Zungu' ${ }^{2}$, J.J. van \\ Oosterhout $^{5,6}$, P.I. Burgess ${ }^{3}$ \\ 'Lions Sight First Eye Unit, Zomba Central Hospital \\ ${ }^{2}$ College of Medicine, University of Malawi, Malawi \\ ${ }^{3}$ Department of Eye and Vision Science, University of Liverpool, UK \\ ${ }^{4}$ Vision Quest Biomedical, Albuquerque, NM, USA \\ ${ }^{5}$ Partners in Hope, Lilongwe, Malawi \\ ${ }^{6}$ David Geffen School of Medicine, Division of Infectious Diseases, University \\ of California, USA
}

DESIGN. Prospective study of the accuracy of a screening test

PURPOSE. Most Malawians with diabetes live in rural areas where access to screening for retinopathy is very 
limited. We investigated the accuracy of an automated grading algorithm used with a hand-held fundus camera for detection of sight threatening diabetic retinopathy that has potential for use in rural settings in low income countries.

METHODS. Subjects were systematically sampled from a single primary care diabetes clinic at Zomba Central Hospital, Malawi, between December 2018 and March 2019. Digital fundus photography was performed through dilated pupils by a health worker using a hand-held camera (Pictor Plus, Volk, OH). Retinopathy was graded in 2 standard fields (disc centred and macula-centred) in 1 eye. An algorithm (Eye Star, Vision Quest, NM USA) graded images as "non-refer" or "refer" corresponding to International Clinical Diabetic Retinopathy Severity Scales level 4 or exudates within 1 disc diameter of the centre of the fovea. Reference standard, feature-specific grading was undertaken by a single certified grader.

RESULTS. Amongst 119 participants, 115 (97\%) were gradable by the human grader; EyeStar software processed $105(88 \%)$. Prevalence of retinopathy grades from manual grading was 'level 0 ' $72 \%$ (95\%CI 63-80), 'level 1' 2\%(0.5-7), 'level 2' 22\%(15-30), 'level 3' 2\%(0.5-7), level 4 0.8\%(0.2-5), level 5 0\%. 12/115 (10\%) subjects exhibited exudates within 1 disc diameter of the centre of the fovea. EyeStar software reported a refer result for 13/105 cases $(12 \%)$. Using a comparable threshold the manual grader referred 19/105 (18\%). Against the reference standard of manual grading, the algorithm achieved sensitivity and specificity for referable retinopathy of $84.6 \%(95 \% \mathrm{CI}$ 55-98) and 91.3\% (84-96), respectively.

CONCLUSIONS. EyeStar software was able to process a high proportion of images from a hand-held camera in a population with limited access to health services. Encouraging sensitivity and specificity data suggest this system has potential to be an effective point of care test in low-resource settings.

\section{Testing of Thirteen Artificial Intelligence Algorithms for Diabetic Retinopathy using Fundus Images from Finland}

P. Huhtinen', N. Hautala ${ }^{2,3}$

'Optomed Plc, Oulu, Finland

2University of Oulu, Department of Ophthalmology, PEDEGO Research Unit and Medical Research Center, Oulu, Finland

${ }^{3}$ Oulu University Hospital, Oulu, Finland

DESIGN. Retrospective study

PURPOSE. Diabetic retinopathy (DR) screening is an essential part of the management of diabetes. Unfortunately, screening is not accessible and affordable to all patients with diabetes. Various solutions, e.g. telemedicine and portable screening solutions, have been proposed to help to solve the issue. Now, artificial intelligence (AI) -based analysis systems have been added to the equation, and they seem to provide additional value. We tested the performance of thirteen different AI algorithms to assess their potential in the screening for DR.

METHODS. The fundus image set of 157 patients with either type 1 or type 2 diabetes, attending screening or follow-up of DR was collected with handheld fundus camera Optomed Aurora and graded as described by Kubin et al. (doi: 10.1111/aos.14850). The images were analysed using thirteen different AI algorithms for DR. The analysis was done with all algorithms without any premodifications of the data set. Moreover, the analysis was blinded. The AI-based analysis results were compared with the human interpretation. When applicable; agreement, sensitivity, and specificity were calculated between the different AI results and ophthalmologist's grading.

RESULTS. Majority of the algorithms provided eye-specific results but there were a few algorithms that returned patient-specific results only. The agreement between the human interpretation and AI results varied from 53\% to $91 \%$. Sensitivities and specificities were $47 \%-90 \%$ and $33 \%-98 \%$, respectively. One of the algorithms was not able to read majority of the images and the performance parameters were not calculated.

CONCLUSIONS. The results show significant differences in performance between algorithms even though all the tested algorithms had been informed to meet typical DR screening performance requirements. Similar results were reported recently by Lee et al. (doi $10.2337 / \mathrm{dc} 20$ 1877). In our opinion the difference in performance in this study is not that crucial. However, it is very important to acknowledge and understand that algorithms must be validated properly in the real-world settings before applying in the routine screening programs.

\section{POSTER SESSION}

\section{Optimization of Immunofluorescent Detection of Vascular Markers on Frozen Human Retinal Sections}

N. Bakker', I. Klaassen', C.J.F. Van Noorden ${ }^{1,2}$, R.O.

Schlingemann'

'Department of Ophthalmology, Amsterdam University Medical Center, The Netherlands

${ }^{2}$ Department of Genetic Toxicology and Cancer Biology, National Institute of Biology, Slovenia

DESIGN. Optimization of immunohistochemical protocol PURPOSE. With this comparative study, we aimed to optimize the immunofluorescence protocol in cryostat sections for visualizing vascular markers in the human retina. Detection of vascular markers is important to study protein 
alterations with spatial context in the blood-retinal barrier during diabetic retinopathy.

METHODS. We extensively compared different conditions for key parameters in the immunofluorescence dual-staining procedure for vascular markers. Indirect fluorescence IHC was performed on sections of frozen human post-mortem retinal tissue. We compared different fixation methods, blocking buffers, antibody concentrations, primary antibody incubation time and temperature and antibody solvents to achieve the highest signal-to-background ratio. For neuron-glial antigen 2 (NG2), stained sections were post-treated with Sudan Black B in an attempt to reduce unspecific staining in nuclei. IHC was performed on serial cryostat sections using primary antibodies against various vascular markers: tight junction marker claudin-5, endothelial cell marker CD31, alpha smooth muscle actin $(\alpha-S M A)$, pericyte marker NG2 and basal lamina markers laminin and collagen type IV.

RESULTS. Paraformaldehyde (PFA) provided the most efficient tissue fixation. Blocking sections with 5\% normal goat serum and $1 \%$ Triton X-100 in PBS gave the best results. An optimal concentration was found for every primary and secondary antibody. Overnight incubation of primary antibodies at $4^{\circ} \mathrm{C}$ rather than three-hour incubation at room temperature gave optimal staining. For dual-staining with both claudin-5 and collagen-IV, and NG2 and CD31, the commercially available antibody diluent was superior as antibody solvent, whereas $5 \%$ normal goat serum with $0.2 \%$ Triton X-100 in PBS was superior for laminin and $\alpha$ SMA. Post-treatment with Sudan Black B could not ameliorate specific staining with NG2.

CONCLUSIONS. With the optimized IHC protocol, the stained retina retained excellent tissue structure. In conclusion, a guideline and an optimized immunofluorescence protocol is provided for sets of vascular markers to maximally visualize these antigens in human retinal tissue.

\section{Correlation between the Aqueous Humor Proteome and Severity of Diabetic Macular Edema}

\author{
L.J. Cehofski',2, S. Thineshkumar', J. Grauslund', K. Kojima ${ }^{2}$ \\ 'Department of Ophthalmology, Odense University Hospital, Odense, \\ Denmark \\ ${ }^{2}$ Department of Ophthalmology, Kyoto Prefectural University of Medicine, \\ Japan
}

DESIGN. Retrospective case-control study

PURPOSE. The global protein profile, the proteome, of the aqueous humor has previously been found to correlate with severity of macular edema in retinal vascular disease. However, the correlation between the aqueous proteome and severity of diabetic macular edema (DME) remains largely unelucidated. Proteomic analysis of DME may bring novel insights into disease mechanisms underlying the complication.

METHODS. Aqueous humor samples from treatmentnaïve patients with DME $(\mathrm{n}=15)$ and age-matched controls $(\mathrm{n}=15)$ were donated from the biobank of Kyoto Prefectural University Hospital, Kyoto, Japan. The study was approved by the Institutional Review Board of Kyoto Prefectural University of Medicine (permission RBMR-C-864-6) and adhered to the tenets of the Helsinki Declaration. The inclusion criterium was macular edema caused by diabetes mellitus. Exclusion criteria were macular edema due to other retinal or chorioretinal disease, use of eye drops three months prior to sample collection, iris rubeosis and glaucoma. Control samples were from age-matched patients from whom aqueous humor samples were obtained prior to cataract surgery. Patients in the control group had no other ocular disease than cataract. The samples were prepared with a filter-aided method and analyzed with label-free liquid chromatography tandem mass spectrometry. Statistical analysis was performed with student's t-test and correction for multiple hypothesis testing with the BenjaminiHochberg method. Proteins were considered statistically significantly changed if $\mathrm{p}<0.05$ with a false-discovery rate at 0.05 . Correlations between severity of macular edema and significantly regulated proteins were calculated with Pearson's correlation coefficient.

RESULTS. The sample preparation and mass spectrometry allowed for quantitative analysis in three analytical runs, reducing variation in quantitative values. In the first analytical runs, 1403 proteins were successfully identified in the aqueous humor. The analyses are ongoing and validation of key proteins is expected to be performed with enzyme-linked immunosorbent assay.

CONCLUSIONS. Advanced proteomic techniques by mass spectrometry can be used to provide in-depth analysis of aqueous proteins. Protein changes discovered with proteomics may bring insights into the complex biological processes underlying DME.

\section{Identifying Undetected Prevalent Disease: The First-Pass Effect in Diabetic Retinopathy Screening Programs}

R. Salongcay 1,2, L.C. Aquino', C.M. Salva', J.K. Sun ${ }^{3,4}$, L.P. Aiello $^{3,4}$, T. Peto ${ }^{2}$, P.S. Silva ${ }^{1,3,4}$

'Philippine Eye Research Institute, University of the Philippines, Manila, Philippines

${ }^{2}$ Centre for Public Health, Queen's University Belfast, Belfast, UK

${ }^{3}$ Beetham Eye Institute, Joslin Diabetes Center, Boston, Massachusetts, USA

${ }^{4}$ Department of Ophthalmology, Harvard Medical School, Boston, Massachusetts, USA

DESIGN. Comparative cross-sectional evaluation of the rates of diabetic retinopathy (DR) 
PURPOSE. To compare the rates of referable DR (refDR) identified on a rapid assessment avoidable blindness and DR (RAAB+DR) survey with community-based DR screening program (DRSP) in the same population.

METHODS. The RAAB+DR survey was performed following standard methodology using random compact segment sampling with the addition of ultrawide field imaging (UWFI) to assess DR and diabetic macular edema (DME). The DRSP was performed using a validated methodology of 5-field 50-degree mydriatic retinal imaging (disc, macula-centered, superior, inferior, temporal) that has been shown to have substantial levels of agreement for DR/DME $(\mathrm{Kw}=0.79 / 0.81)$ compared to standard Early Treatment Diabetic Retinopathy Study (ETDRS) photography. The RAAB+DR was performed from August to October 2017 in preparation for the implementation of the DRSP. The DRSP is ongoing and the data presented was collected from February to March 2021 and represent 10.6\% of the screening target. RefDR was defined as moderate nonproliferative DR (NPDR) or worse, any DME or ungradable images.

RESULTS. Evaluating the same target population, a total of 1,609 individuals were evaluated in the RAAB $+\mathrm{DR}$, with $341(21.2 \%)$ individuals with diabetes (PwDM). DR severity based on UWFI was 239(70.1\%) no DR, 38(11.1\%) mild NPDR, 35(10.3\%) moderate, 10(2.9\%) severe, 19(5.6\%) proliferative DR (PDR), 34(7.4\%) with DME and $9(2.4 \%)$ were ungradable. The DRSP evaluated $562 \mathrm{PwDM}$, distribution of DR severity was 335(59.6\%) no DR, 92(16.4\%) mild NPDR, 51(9.1\%) moderate, $39(6.9 \%)$ severe, 33(5.87\%) PDR, 30(5.4\%) with DME and $31(5.5 \%)$ were ungradable. RefDR was identified in $86(18.7 \%)$ of the RAAB+DR cohort and $169(30.1 \%)$ in the DRSP.

CONCLUSIONS. In the initial year of screening there will be a significant first-pass effect, detecting higher levels of previously undetected prevalent disease. Due to this first-pass effect, the planning of DRSP needs to account for this initially large demand placed on eye care service. In this cohort, there was $61 \%$ increase in the rate of refDR that will need to be accommodated in the existing health care system.

\section{Five Year Outcomes of Diabetic Eye Screening in Patients Aged 80 Years}

N. Albutt ${ }^{1,2}, \mathrm{H}$. Wharton ${ }^{1,2}$, S. Jacobs ${ }^{1,2,3}$

'Birmingham, Solihull and Black Country Diabetic Eye screening programme, Birmingham, UK

${ }^{3}$ University Hospitals Birmingham, Birmingham, UK

${ }^{4}$ Aston University, Aston, UK

DESIGN. Retrospective audit

PURPOSE. To assess the incidence of referable diabetic retinopathy (DR) in patients in their 80's looking at rates of progression of disease and determine whether screening interval can be extended safely in this age group.

METHODS. Patients who were aged 80 when they attended screening during April 2014-March 2015 were included. Screening results at baseline and over the next four years were analysed along with the patient demographics. Referable DR was classed as maculopathy, preproliferative or proliferative changes.

RESULTS. 1880 patients aged 80 were included. Average age at their first ever screen was 73 years. 51\% were noted to be male and the majority were Caucasian $68 \%$, with $21 \%$ Asian and $11 \%$ Black. At 80 years (baseline screen) $26(1.4 \%)$ had referable DR, 2 required treatment. At year 1 follow up (FU) 1722 patients were screened of these 12 $(0.7 \%)$ had referable DR with only 1 requiring treatment. At year $2 \mathrm{FU}, 1347$ were screened of which $18(1.3 \%)$ had referable DR, 4 received treatment. At year $3 \mathrm{FU}, 1538$ patients were screened, $11(0.7 \%)$ were referred for DR and 3 had treatment. At year 4 FU, 1119 were screened, 9 $(0.8 \%)$ had referable DR and 1 received treatment. In total $76(4 \%)$ were referred to the hospital eye service (HES) for DR over the FU period, of which $11(0.6 \%)$ received treatment. Over the course of the FU, 403 (21\%) died.

CONCLUSIONS. This study showed that the risk of progression of retinopathy is quite low in this elderly age group and only a small proportion of patients developed referable retinopathy requiring any treatment. It is possible to consider increasing screening interval in this 80 plus cohort in line with current national recommendation to increase the screening interval in people with low risk of sight loss. Screening attendance rates were found to decrease significantly with advancing age due to reasons such as death and other diseases making them no longer suitable for screening.

\section{A Qualitative Study on Barriers and Enablers to Uptake of Diabetic Retinopathy Screening by People with Diabetes in the Western Province of Sri Lanka}

M.M.P.N. Piyasena ${ }^{1,2.3}$, G.V.S. Murthy ', J.Y.L. Yip', C. Gilbert ', T. Peto ${ }^{3}$, M. Premarathne ${ }^{4}$, M. Zuurmond'

'London School of Hygiene and Tropical Medicine, London, UK

${ }^{2}$ National Eye Hospital, Colombo, Sri Lanka

${ }^{3}$ Ministry of Health, Sri Lanka

${ }^{4}$ Centre for Public Health, Queen's University Belfast, Belfast, UK

DESIGN. Qualitative Study

PURPOSE. Blindness and visual impairment from diabetic retinopathy (DR) are avoidable through early detection and timely treatment. The Western province of Sri Lanka has the highest prevalence of diabetes mellitus (DM) in the country. A situational analysis identified a significant gap in DR screening services (DRSS) uptake. 
Barriers that hinder people with DM (PwDM) from attending DRSS are poorly understood. The purpose of this study is to understand the factors which influence uptake of DRSS and follow up to inform health promotion strategies and improve uptake of these services.

METHODS. Eleven focus group discussions (FGDs) were conducted with PwDM who presented to medical and eye clinics in three public sector institutions (tertiary and secondary level) in the Western province between October 2016 and March 2017. We enrolled six groups (4 Sinhala speaking, 2 Tamil) of women and five groups (3 Sinhala and 2 Tamil) of men representing ethnicity and gender. We performed a thematic analysis and described the main themes and subthemes using the socio-ecological model as a framework.

RESULTS. We identified lack of knowledge of both, the condition and the need for screening as key barriers to access DRSS. Socio-cultural factors in the family environment, economic reasons and institutional factors were also important barriers. Additional reasons include long waiting time at eye clinics and poor referrals exacerbated by the lack of a systematic DRSS. In addition, attitudes to DRSS such as fear of discomfort from the procedure and the need for accompaniment following mydriasis were also deterrents to follow up screening.

CONCLUSIONS. This study has shown that there are inter-related user, family and institutional factors which affect the uptake of DRSS. Understanding how DR is conceptualised by PwDM in this region is essential to refine strategies to improve access to DRSS. Strategies to improve knowledge need to be more culturally acceptable and relevant to PwDM and their families, with increased availability of DRSS at convenient locations may increase timely uptake of screening.

\section{Inclusion of Diabetic Retinopathy Screening Strategies in National Level Diabetes Care Planning in Low and Middle-Income Countries: A Scoping Review}

K. Curran', P. Piyasena' ${ }^{1,2}$, N. Congdon ${ }^{1,3,4}$, L. Duke ${ }^{5}$, B. Malanda ${ }^{5}$, T. Peto'

'Centre for Public Health, Queen's University Belfast, UK

${ }^{2}$ Directorate of Policy Analysis and Development, Ministry of Health, Sri Lanka

${ }^{3}$ Zhongshan Ophthalmic Centre, Sun Yat-sen University, Guangzhou, China ${ }^{4}$ Orbis International, New York, USA

${ }^{5}$ International Diabetes Federation, Brussels, Belgium

DESIGN. Scoping review

PURPOSE. Diabetes is a major public health concern with approximately $80 \%$ of the burden falling on low- and middle-income countries (LMICs). Diabetic retinopathy (DR) is one of the most common microvascular complications of diabetes and early detection through diabetic retinopathy screening programmes (DRSPs) is essential to prevent vision impairment and blindness. Careful planning at a national level is crucial for effective implementation of such programmes. The main purpose of this study was to identify LMICs that have not included DR in their national diabetes strategic plans, action plans or policies, or as part of their non-communicable diseases policies or prevention of blindness plans. Furthermore, the study aimed to assess gaps in national level DR services planning in LMICs.

METHODS. The scoping review protocol has been previously published. Data were collected from databases and searches, including grey literature. Consultations with key informants from LMICs were conducted to strengthen the findings. A policy-framework with four stages: agenda setting (early and late phases), policy formation, policy implementation and evaluation was adopted to address gaps in national level DR service planning, and a colourcoded system highlighted country progress in terms of their development in the policy cycle. A quantitative analysis was carried out to map the data in tabular form, highlighting country progress in terms of national level DRSP implementation.

RESULTS. Lower-middle (29/50, 58\%) and upper-middle income countries $(27 / 59,45.8 \%)$ are making more progress than low-income countries $(4 / 29,13.8 \%)$ in term of DR policy planning. However, no surveyed countries have implemented national DR policies. Upper-middle income countries are making the most progress in implementing national DRSPs; though, their progress remains slow, with only $5 / 59(8.5 \%)$ having such programmes.

CONCLUSIONS. There are significant gaps in the literature, with no data reported in 78/138 (56.5\%) LMICs. Further research is necessary to support and document DR policy development and implementation in LMICs for the future.

\section{Out of Hours Diabetic Eye Screening - A High Risk Group}

R. Aldred', S.J. Aldington ${ }^{2}$, I.M. Stratton ${ }^{2}$

'Gloucestershire Diabetic Eye Screening Programme, Gloucestershire Hospitals NHS Foundation Trust, UK

${ }^{2}$ Gloucestershire Retinal Research Group, Gloucestershire Hospitals NHS Foundation Trust, UK

DESIGN. Gloucestershire Diabetic Eye Screening Programme has 35,028 patients invited annually for mydriatic 2-field digital imaging. Attendance rates are lower in younger people of working age. Out-of-hours [OOH] (evenings, weekends) clinics are run to enable them to attend. We report here on screening outcomes from $\mathrm{OOH}$ and compare them with those from the routine weekday clinics. Data was exported from the screening programme database from $01 / 01 / 2016$ to $31 / 12 / 2019$. 
PURPOSE. Investigate referral rates in $\mathrm{OOH}$ and standard working hours and relationship with demographic factors. METHODS. Retinal images were captured and graded following the English NHS Diabetic Eye Screening Programme system. Outcomes were urgent referral for Diabetic Retinopathy [DR], routine referral for DR, urgent referral for non-DR, routine referral for non-DR, referral to Digital Surveillance [DS], referral for slit lamp biomicroscopy [SLB] if unassessable, or mild DR or no DR (both routine rescreen). Data for $\mathrm{OOH}$ and standard working hours clinics was exported from clinical systems and matched with other available information.

RESULTS. Over the 4 years, 224 of 4,596 screening episodes in $\mathrm{OOH}(4.9 \%)$ led to referrals to the hospital eye service (HES): 20 (0.4\%) urgent DR, $32(0.7 \%)$ routine DR, 15 $(0.3 \%)$ urgent non-DR, $23(0.5 \%)$ routine non-DR, and 134 $(2.9 \%)$ to digital surveillance. 17 screening episodes $(0.02 \%)$ were referred to SLB. The comparator data for weekday working hours appointments over the 4 years, 3,851 of 93,559 people $(4.1 \%)$ were referred to HES: $216(0.2 \%)$ urgent DR, $623(0.7 \%)$ routine DR, $218(0.2 \%)$ urgent nonDR, $404(0.4 \%)$ routine non-DR, and 2,390 (2.6\%) to digital surveillance. 1,916 screening episodes $(2.15 \%)$ were referred to SLB. There was a significant trend to more severe DR in the $\mathrm{OOH}$ group ( $\mathrm{p}=0.012$ on Mantel Haenszel test).

CONCLUSIONS. OOH clinics provide more convenient attendance opportunities for working people. Those attending these clinics have more DR and more serious DR than those who attend in working hours. Monday to Friday 9 to 5 clinics do not give equal opportunities for all at-risk groups to attend for screening.

\section{Diabetic Retinopathy in Pregnancy: Screening Attendance Rates and Development of Disease}

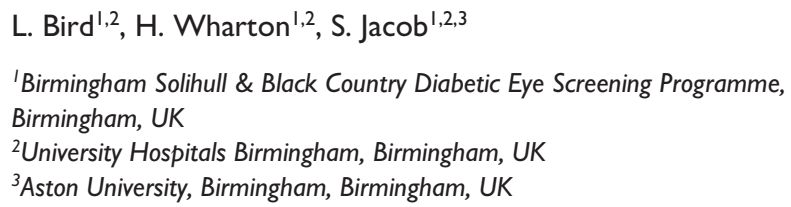

DESIGN. Retrospective cohort study.

PURPOSE. To assess diabetic eye screening attendance records of patients throughout their pregnancy and to identify the development and progression of diabetic retinopathy pre, during and post pregnancy.

METHODS. Patients who went full term with expected delivery dates between June 2018 and May 2019 were included. Demographics, retinopathy grades and outcomes were recorded from the last screen pre-pregnancy, during and the first screen post pregnancy.

RESULTS. 97 women with an average age of 32.7 (19-44) years. The majority were Asian 49, with 32 white, 9 Black and 7 other.
Average gestation of first screen during pregnancy was 17.5 (4-33) weeks. 64 had no DR, 25 background changes, 7 maculopathy and 1 unassessable. 11 were screened during third trimester and all returned to annual recall (AR). 6 were referred to ophthalmology. Sixty-six attended a second screen with an average gestation of 27.4 (10-36) weeks. Compared to first screen 53 were stable, 6 improved and 7 deteriorated. One was referred urgently for pre-proliferative changes and diabetic maculopathy. Nine patients attended 3 pregnancy screenings, average gestation 31.3 (28-35) weeks. 6 were stable, 2 had improved and 1 had deteriorated. Looking at pre- and post-pregnancy, 68 attended at least once prior averaging 12.4 (2-113) months. Comparing the most recent pre-pregnancy screen to first pregnancy screen, 49 had the same results, 3 improved, and 16 deteriorated. Fifty attended post-partum after an average of 13.5 (6-31) months. 39 of these showed stable results since their final pregnancy screen, 8 improved, 3 deteriorated. One had preproliferative changes and was referred urgently. Fourteen only ever attended screening during their pregnancy, 35 attended all screens pre-, during and post-pregnancy.

CONCLUSIONS. The results of this audit suggest that retinopathy status remained fairly stable in the majority $(94 \%)$ and only a minority developed referable disease $(6 \%)$ during early pregnancy. Most cases improved or remained stable towards the end of gestation. Outcomes for patients referred to eye clinic suggest that referable maculopathy noted only needed observation and no treatment suggesting milder disease and improvement. As pregnancy progresses, non-attendance was noted to increase significantly.

\section{Progression of Diabetic Retinopathy in Type $I$ and 2 Diabetes During Pregnancy and Postpartum in the Danish National Diabetes Retinopathy Screening Program}

J.E. Larsson', L. Stokholm ${ }^{2,3}$, T. Bek ${ }^{4}$, N. Andersen ${ }^{5}$, J. Grauslund $^{2,6,7}$, C.S. Laugesen', on behalf of the OASIS Study Group

'Department of Ophthalmology, Zealand University Hospital Roskilde, Roskilde, Denmark

${ }^{2}$ Department of Clinical Research, University of Southern Denmark, Odense, Denmark

${ }^{3}$ OPEN - Open Patient data Explorative Network, Odense University Hospital, Odense, Denmark

${ }^{4}$ Department of Ophthalmology, Aarhus University Hospital, Aarhus, Denmark

${ }^{5}$ Organization of Danish Practicing Ophthalmologists, Copenhagen, Denmark ${ }^{6}$ Department of Ophthalmology, Odense University Hospital, Odense, Denmark

${ }^{7}$ Steno Diabetes Center Odense, Odense University Hospital, Odense, Denmark

DESIGN. Registry-based, national cohort study.

PURPOSE. Pregnancy in diabetes can increase the risk of progression of diabetic retinopathy (DR). This national, 
retrospective registry-based cohort study aims to identify women with diabetes type 1 (DM1) and 2 (DM2) with progression of DR during pregnancy or postpartum comparing them with a control group.

METHODS. Diabetic retinopathy screening is nationally implemented in Denmark and the results are registered in the Danish Registry of Diabetic Retinopathy (DiaBase) using the 5-step ICDRDS scale. Baseline DR was the last screening 12-24 months before pregnancy. Progression of DR was registered in all trimesters and 12 months postpartum. The control group consisted of women who did not give birth and were screened twice in the study period.

RESULTS. We identified 1327 parous women aged 18 to 50 , with a total of 1509 births, and a control group of 2359 nulliparous women aged 20 to 40, all screened for DR in 2013-2018.

In the DM1 study group 576 women (55.0\%) had no DR at baseline versus $929(63.9 \%)$ in the control group. At baseline 471 women $(45.0 \%)$ had DR level $1-4$ in the study group versus 525 (36.1\%) in the control group. At the postpartum follow-up 539 women (51.5\%) had DR versus 736 $(50.6 \%)$ in the control group at follow-up.

In the DM2 study group 432 women (91.5\%) had no DR at baseline versus $829(91.6 \%)$ in the control group. At baseline 40 women $(8.5 \%)$ had DR level $1-4$ in the DM2 study group versus $76(8.4 \%)$ in the control group. At the postpartum follow-up 47 (10.0\%) had DR versus 125 women (13.8\%) in the control group at follow-up.

In the study group 2338 eyes showed no progression of DR, $62 \%$ having DM1 and 38\% DM2. Progression was mainly seen in the third trimester and postpartum, $87 \%$ having DM1.

CONCLUSIONS. Higher DR-level at baseline and DM1 were associated with DR progression during pregnancy and postpartum. Parous women had a lower incidence for progression of DR compared to nulliparous women.

\section{Treatment and Follow Up of Patients Graded as R3 (Proliferative Retinopathy) by Diabetic Retinopathy Screening Programme (DRSP) In Belfast, Northern Ireland}

\author{
A. Heaney', D. McCullagh', N. Parker', T. Peto ${ }^{1,2}$ \\ 'Belfast Health and Social Care Trust, Belfast, UK \\ ${ }^{2}$ Centre for Public Health, Queen's University Belfast, Belfast, UK
}

DESIGN. Retrospective audit of patient records.

PURPOSE. This study assessed the treatment received by patients graded as R3 by DRSP in Belfast. The data enabled identification of trends which facilitated the implementation of treatment pathways. In the last 5 years the DRSP in Belfast has undergone modernisation and it is vital to ensure that patients are being followed up in an appropriate time interval and that these $\mathrm{R} 3$ patients are not lost to follow up. It has enabled us to assess if Belfast is meeting the targets set by the Royal College of Ophthalmologists, and identify any areas for improvement.

METHODS. Patient records were reviewed over a 1 year period (October 2015 to September 2016) of all patients graded as R3 at diabetic retinopathy screening in either eye in Belfast.

RESULTS. 946 patients graded as R3 in at least 1 eye, median age 62 range $18-91,70 \%$ male and $30 \%$ female. Of the 946 patients, 746 (79\%) remained within the screening programme with inactive disease, $46(5 \%)$ did not attend follow up and 154 (16\%) were lost to follow up.

Only 370 patients required treatment for having active disease; $239(65 \%)$ treated with laser alone, $50(14 \%)$ patients had intravitreal antiVEGF injection alone, $73(20 \%)$ had laser and injection and 8 patients required other interventions including VR surgery. Of the patients who required laser treatment, $383(84 \%)$ required 1-5 laser sessions, 60 (13\%) required 6-10 laser sessions and $12(3 \%)$ had 11-15 laser sessions. Of the patients who required intravitreal anti VEGF injections, $92(55 \%)$ had 1-5 injections, 36 (21\%) had 6-10 injections, 24 (14\%) had 11-15 injections, $16(10 \%)$ had 16-30 injections.

CONCLUSIONS. A large majority of patients graded as $\mathrm{R} 3$ could remain within the screening programme and were not lost to follow up. Many of these patients require treatment, emphasizing the importance of follow up and the development of pathways to help deliver this service to all patients who require it.

\section{Diabetic Retinopathy Screening in Individuals with Severe Mental IIIness}

J. Sandford ${ }^{1,2}$, Z.S. Sherwood', S.J. Jacob ${ }^{2}$

'Birmingham, Solihull and Black Country Diabetic Eye Screening Programme, Birmingham, UK

${ }^{2}$ Birmingham and Solihull Mental Health NHS Foundation Trust, Birmingham, UK

DESIGN. A collaborative data sharing project between two NHS Trusts, working together to identify ways of improving attendance and access to screening for patients with Severe Mental Illness (SMI).

PURPOSE. The goals were to increase the attendance rate of diabetic retinal screening in those diagnosed with SMI by increasing the number of patients who receive a screening invite within this cohort. Another aim was to encourage earlier treatment intervention to prolong quality of sight in this population group, as well as identifying any possible barriers to accessing annual diabetic retinal screening.

METHODS. A list of patients with both SMI and diabetes was identified which was then categorised and analysed. Interventions were made to ensure all appropriate patients 
were registered and offered screening. The data was analysed initially before interventions were attempted, with a second analysis performed six months later to assess the effectiveness of these efforts.

RESULTS. Screening attendance rates in this cohort increased by $31 \%$ (28/91) by the end of data run 1 and $28 \%$ $(30 / 109)$ by the end of data run 2 . A significant number of these patients $(17 \%$ and $21 \%$ respectively in each data run) who were screened, required onward referral to hospital eye services. In the Not Registered patient group, $84 \%$ of those with confirmed diagnosis in data run 2 became registered and offered screening. The number of individuals registered with DESP at the six month data review increased from $35 \%$ for the first data collection to $84 \%$ in the second. As the project progressed, the processes became more streamlined and results improved once the method had been firmly established.

CONCLUSIONS. These results highlighted how information sharing and collaborative working between services has the potential to improve long term patient health outcomes, as it can increase the number of those eligible individuals with SMI registered for diabetic retinopathy screening. This improves attendance, thus improving long term eye health outcomes. The project has shown the potential for future data sharing collaborations between NHS providers, as well as highlighting the need for further improvement, development and investment to target inequalities and improve access for all.

\section{Diabetic Eye Screening Outcomes after Non- Attendance for Three or more Years}

\author{
L. Brown ${ }^{1,2}$, L. Bird ${ }^{1,2}$, S. Jacob ${ }^{1,2,3}$ \\ 'Birmingham, Solihull \& Black Country Diabetic Eye Screening Programme, \\ Birmingham, UK \\ ${ }^{2}$ University Hospitals Birmingham NHS Foundation Trust, Birmingham, UK \\ ${ }^{3}$ Aston University, Birmingham, UK
}

DESIGN. A retrospective audit of patients who attended screening following three plus years of no response, including patients never screened though registered.

PURPOSE. To analyse the outcomes of these patients to determine if delay in screening affected levels of diabetic retinopathy (DR) found. To identify the demographics of do not respond (DNR) patients in order to promote effective engagement work thereby improving programme uptake.

METHODS. Retinopathy grades and outcomes were analysed with demographics for patients attending between May 2020 and October 2020 with previous DNR.

RESULTS. 346 patients were analysed- 55\% male, 43\% female and 2\% unknown. Average age was 57 years. Ethnicity was $42 \%$ Caucasian, 35\% Asian, 9\% Black, $8 \%$ other and $6 \%$ unknown. 51 patients had never attended screening, of which 9 (18\%) had been registered for over ten years. Fortunately none of these patients had pre-proliferative or proliferative changes. 14 showed background retinopathy including 5 with maculopathy, where 2 were referred to Ophthalmology and 3 were referred to digital surveillance clinics. The remaining 295 patients that previously attended had an average delay period of 54 months (30-152 months). Comparing previous and most recent grades, 189 remained stable, 15 improved, 89 deteriorated and 2 were unassessable. 7 developed proliferative changes with maculopathy. 12 developed pre-proliferative changes including 7 with maculopathy. 97 showed background retinopathy including 26 with maculopathy. In total, $31(9 \%)$ were referred to ophthalmology for DR, 6 for non-DR, and 1 for urgent non-DR. Of those referred for DR, 25 attended and 10 received treatment. 7 were referred urgently and 5 of them received treatment.

CONCLUSIONS. This audit suggests that patients who attend after years of non-attendance had some deterioration but only $9 \%$ had referable disease and when referred to HES most patients attended. Majority of the patients were made eligible following review at an optician. So non-attendance for screening gets picked up when attending for routine eye tests. The audit also shows that persistent non-attenders are usually males of working age and predominantly of Asian and Caucasian ethnicity which matches the results of other screening engagement work.

\section{Diabetes-Related Blindness between 1999 and 2018 in Denmark}

S.L. Blindbæk', L. Stokholm²,3, S. Heegaard ${ }^{4,5}$, T. Bek ${ }^{6}$, J. Grauslund ${ }^{1,2,7}$, on behalf of the OASIS Study Group

'Department of Ophthalmology, Odense University Hospital, Odense, Denmark

${ }^{2}$ Department of Clinical Research, University of Southern Denmark, Odense, Denmark

${ }^{3}$ Open Patient Data Explorative Network, Odense University Hospital \&University of Southern Denmark, Odense, Denmark

${ }^{4}$ Department of Ophthalmology, Rigshospitalet-Glostrup, Copenhagen, Denmark

${ }^{5}$ Department of Clinical Medicine, University of Copenhagen, Copenhagen, Denmark

${ }^{6}$ Department of Ophthalmology, Aarhus University Hospital, Aarhus, Denmark

${ }^{7}$ Steno Diabetes Center Odense, Odense University Hospital, Odense, Denmark

DESIGN. National, longitudinal, cohort study PURPOSE. The past decades have offered tremendous development within treatment of diabetic retinopathy, but in Denmark it is not known, if this has also affected the number of persons with diabetes-related blindness.

The aim of this study was to calculate the total and disease-specific incidence of blindness in Denmark from 1999-2019. 
METHODS. As part of the Ocular And Systemic complications In diabetic retinopathy Study (OASIS), we performed a national study based on data from approximately 8000 patients living with social blindness and registered in the volunteer-based organization Danish Society of the Blind. Social blindness is defined as visual acuity $\leqslant 35$ Early Treatment Diabetic Retinopathy Study letters on the better eye or similar visual deficits. Cause of blindness is documented by the referring physician/ophthalmologist upon registration.

RESULTS. Preliminary data suggest that for the 5-year periods 1999-2003, 2004-2008, 2009-2013 and 20142018 , the absolute number of new members of Danish Society of the Blind with diabetes as registered cause of blindness was 207, 181, 97 and 44, which represents a decrement of $78.7 \%$ during the 20 years. Correspondingly, diabetes as the registered cause of blindness accounted for $4.6 \%, 4.3 \%, 4.0 \%$ and $2.1 \%$ among all new member of the Danish Society of the Blind.

For members $<65$ years, the corresponding numbers were 104, 100, 63 and 21, which represent a decrement by $77.9 \%$ between $1999-2018$, and diabetes as the registered cause of blindness accounted for $20.9 \%, 20.7 \%, 16.3 \%$ and $7.4 \%$ among all new member of the Danish Society of the Blind. In this age group, diabetes dropped from the second to the fifth most frequent registered cause of blindness between the periods 1999-2003 and 2014-2018.

CONCLUSIONS. Preliminary data suggest that the incidence of diabetes-induced social blindness decreased by approximately $80 \%$ in the period $1999-2019$. To examine the numerous possible reasons behind the pronounced decrease in diabetes-induced blindness, we plan to link data from the Danish Society of the Blind to register data in order to describe the characteristics of blind diabetes patients as compared to those living with diabetes without loss of vision.

\section{A Retrospective Analysis of Factors Affecting Attendance at the Northern Ireland Diabetic Eye Screening Programme in Young People with Diabetes Aged 12-26}

L. Cushley', K. Curran', A. Bell ${ }^{3}$, U. Graham², D. McCance², Q. Wen', T. Peto',2

'Centre for Public Health, Queen's University Belfast, Belfast, UK

${ }^{2}$ Belfast Health and Social Care Trust, Belfast, UK

${ }^{3}$ London North West Healthcare, London, UK

DESIGN. Analysis of factors affecting attendance of young people with diabetes (YPwD), aged 12-26, at Diabetic Eye Screening Programme Northern Ireland (DESPNI), UK

PURPOSE. To investigate attendance rates at DESPNI among young people (12-26 years) with diabetes.
METHODS. This retrospective study collected data for age, gender, hospital area, deprivation score, attendance at screening and diabetes clinics, $\mathrm{HbAlc}$ value and diabetic retinopathy/maculopathy (DR/DMac) grade. Anonymised data were analysed using $\mathrm{R}$ version 3.6.2 and an ordinal logistic regression was carried out.

RESULTS. Altogether, there were $2330 \mathrm{YPwD}$ eligible for the cohort, of these, 499 had to be removed due to missing variables, resulting in a cohort of 1831 for analysis. The cohort included 387 people under 15,489 between 16-29, 417 between 20-22 and 538 aged 23-26, the mean age of the cohort was 19.5. Forty-seven percent were male and $53 \%$ were female. After ordinal logistic regression, age, attendance at diabetes clinic, $\mathrm{HbAlc}$ value, diabetes duration and hospital clinic area were statistically significantly associated with attendance. Results showed that those aged between $12-15$ were $75 \%$ more likely to attend DESPNI than those aged 16-26. YPwD who were diagnosed in the past 5 years were $30 \%$ more likely to attend screening than those diagnosed for over 5 years. In addition, those who attended their diabetes clinic appointments were $89 \%$ more likely to attend DESPNI. In keeping with diabetes clinic attendance and engagement, young people with lower $\mathrm{HbAlc}$ values were $46 \%$ more likely to attend their eye screening. Interestingly, young people in one particular area were $43 \%$ more likely to attend screening than in other hospital areas.

CONCLUSIONS. This study provides further information on factors influencing YPwD annual attendance at DESPNI. This information included the whole cohort of YPwD in Northern Ireland and so it allows detailed analysis of barriers and enablers in order to maximize impact.

\section{Impacts on Eye Health for Patients Who Fail to Attend Hospital Ophthalmology Appointments}

J. Harmon', S. Mc Mahon', C. Murphy³, D. Keegan³, R. Acheson', L. O'Toole ${ }^{2}$

'NEC Care, Cork, Republic of Ireland

${ }^{2}$ ENP Health Solutions, Letterkenny, Republic of Ireland

${ }^{3}$ National Screening Service, Dublin, Republic of Ireland

DESIGN. Retrospective study

PURPOSE. To identify patients who did not attend their scheduled hospital-based ophthalmology clinical appointments, and to determine if the resulting delay impacted on their eye health.

METHODS. This study looked at NEC Care patients from January 2016 to May 2021 who were discharged from treatment centres due to multiple consecutive missed appointments. There are differing discharge protocols between hospital treatment centres, however in general patients are discharged if they fail to attend two consecutive appointments. Patients aged 12 and older were 
included. The geographical area studied encompassed 17 counties within the Republic of Ireland.

RESULTS. We identified 6,600 patients who failed to attend their scheduled hospital ophthalmology appointments over a 66-month period. These patients had been offered at least 2 appointments by the hospital following referral. Of this patient group, $80 \%$ had been referred for further management of their diabetic retinopathy, and 20\% represented referral for non-diabetic eye disease. Due to their non-attendance the mean time between patient referral and subsequent review was 24 months (range 5-57 months). Altogether, $42 \%$ of patients demonstrated a progression in their diabetic retinopathy between initial referral and subsequent examination. Four percent of patients failed to attend despite being referred on three separate occasions to the hospital from screening.

CONCLUSIONS. The impact of non-attendance is not confined to primary diabetic screening but also affects hospital-based clinics. Despite the patient being aware that pathology has been detected at initial screening, 30\% of patients referred to hospital clinics for further management are discharged secondary to non-attendance. Missed appointments not only have a detrimental effect on the patient's ocular health, but they are also a lost opportunity for treatment for others.

\section{Updating a Diabetic Eye Screening Programme - A Long and Winding Road}

I Stratton

Gloucester retinal research group, Cheltenham General Hospital, Gloucestershire, UK

DESIGN. A personal look back on how a new standard was introduced to a national screening programme.

PURPOSE. To try to explain why it took ten years to add a new quality standard to the English Diabetic Eye Screening Programme.

METHODS. A retrospective look at publications and work around this topic.

RESULTS. Around 2010 I attended an English Diabetic Eye Screening Programme meeting. Proportion of those invited for screening who attended each year was a Quality Standard, and was running at about $85 \%$. However, we did not know whether the $15 \%$ who did not attended were the same $15 \%$ each year or whether they were the same $15 \%$. At the meeting I suggested that we count how many people on the register for 3 years had not been seen at all in the three years. This wasn't accepted. However, we knew that those who did not attend for 3 years were at higher risk. In order to add to the evidence base we used data from the Four Nations Study to examine the risk after different periods from diagnosis of diabetes with no attendance, paper published in 2015. The odds ratio for urgent referral to ophthalmology was 4.3 (odd ratio 2.6 to 7.1 ). We know that some local programmes were making Herculean efforts to screen those who had not recently attended and that the unpublished results showed that the research work was extremely robust. However, without more published evidence it took until 2019 until the DES-S08 Quality Standard was introduced (proportion of cohort who have DNA'd in previous 3 years) and now (2021) it is to become a Key Performance Indicator. Those who did the audits around focussing resources on improving attendance in those unscreened said that "lack of time" was the reason for not writing them up.

CONCLUSIONS. More timely changes to screening programmes might be possible if resources were directed to reporting the impact of local initiatives to improve diabetic eye screening programmes.

\section{Diabetic Retinopathy and Risk of Prevalent and 5-Year Incident Cardiovascular Disorders in Type 2 Diabetes}

L. Stokholm ${ }^{1,2}$, K.H. Frederiksen ${ }^{2,3}$, K.H. Rubin ${ }^{1,2}$, R. Kawasaki ${ }^{5}$, J. Grauslund ${ }^{2,3,4}$, on behalf of the OASIS Study Group

'OPEN Patient data Explorative Network(OPEN), Odense University Hospital, Odense, Denmark

${ }^{2}$ Department of Clinical Research, University of Southern Denmark, Odense, Denmark

${ }^{3}$ Department of Ophthalmology, Odense University Hospital, Odense, Denmark

${ }^{4}$ Steno Diabetes Center Odense, Odense University Hospital, Odense, Denmark

${ }^{5}$ Department of Vision Informatics, University of Osaka, Osaka, Japan

DESIGN. A matched, register-based cohort study PURPOSE. In earlier studies, type 2 diabetes and diabetic retinopathy (DR) have been associated with cardiovascular disorders (CVD), but longitudinal data are sparse, and it is not known if correlations would also apply in an entire population of patients, of whom many do not attend hospital care

METHODS. This study is a nationwide, matched, register-based cohort study where cases $(n=153,238)$ were identified in the Danish Registry of Diabetic Retinopathy (DiaBase) as patients with type 2 diabetes participating in the national DR screening programme between 20132018. Each case were matched $1: 5$ by sex and birth year with non-diabetes controls $(n=746,148)$. Index date was defined as the date of the first occurrence in DiaBase just as level of DR was assessed at the first occurrence in DiaBase and according to the International Clinical Diabetic Retinopathy Severity Scale (level 0-4 according to worse eye). Data on CVD (cardiac, neurological and peripheral vascular disorders) were identified from the Danish National Patient Register. At the date of first DR screening for cases, the risk of CVD was estimated in a 
multivariable logistic regression model, and multivariable Cox proportional hazard models were used to estimate the risk of CVD within five years.

RESULTS. We found that $31.9 \%$ of cases and $21.1 \%$ of the controls were registered with CVD at index date. Those registered with CVD were more likely to be men, older of age and more likely to be widowed or divorced than those without CVD. Overall, patients with type 2 diabetes had a higher risk of prevalent (multivariable adjusted OR: $1.71,95 \%$ CI 1.69-1.73) and a 5-year incident CVD (multivariable adjusted HR: 1.23 95\% CI 1.21-1.26) with a trend of increased risk with higher level of DR (multivariable adjusted HR 1.20 vs 1.41 vs 1.69 vs 2.33 vs 2.38 for DR-level 0 to 4 , respectively).

CONCLUSIONS. In Denmark, patients with type 2 diabetes had a higher risk of prevalent and incident CVD. Further, we found a dose-response gradient between DR and CVD, indicating an association between DR and CVD.

\section{Characteristic Features of Diabetic Retinopathy (DR) In UK Biobank (UKBB)}

M.J. Quinn', T. Peto', NetwORC UK Graders², S.

Madhusudhan ${ }^{3}$, K. Balaskas ${ }^{4}$, UKBB Eye and Vision

Consortium $^{5}$, P. Foster ${ }^{6}$

'Centre for Public Health Queen's University Belfast, UK

${ }^{2}$ NetwORC UK, UK

${ }^{3}$ St Paul's Eye Unit, Liverpool, UK

${ }^{4}$ Moorfields Eye Hospital NHS Foundation Trust, UK

${ }^{5}$ UK Biobank, UK

${ }^{6}$ Institute of Ophthalmology, University College London, UK

DESIGN. UKBB is a biomedical database comprising lifestyle, biomedical and genetic data from over 500,000 UK participants aged 40-69. Almost 70000 participants had ophthalmic imaging of colour fundus photography and optical coherence tomography (OCT) at baseline.

PURPOSE. To provide human grading results in order to describe the characteristic features of DR on colour and OCT imaging in UKBB.

METHODS. Colour and OCT images were analysed by trained and certified NetwORC UK graders to record relevant DR features visible on images. These data were captured in a study specific grading form linked to a study database. Quality assurance was carried out at a rate of 1:20 gradings.

RESULTS. Approximately $99 \%$ of colour images and $99 \%$ of OCT images were available for both eyes. Of these available images, $90 \%$ of colour images and $99 \%$ of OCT images were found to be of gradable quality. Abnormalities were found on approximately $36 \%$ of participant images. Microaneurysms and haemorrhages were found in $1.04 \%$ of UKBB participants. An additional $0.08 \%$ were found to have laser treatment with inner retinal changes that might be suggestive of diabetic eye disease. Potential diabetic eye changes, such as cotton wool spots and exudates were graded in an additional $0.09 \%$ of participants. Amongst those with ungradable colour images but gradable OCT images, $0.32 \%$ of participants were found to have OCTabnormality, such as inner retinal changes, which are comparable to characteristic changes typically found in diabetic eye disease.

CONCLUSIONS. The cohorts identified with characteristic features of DR will form the basis of further analysis for genetic and lifestyle determinants. The importance of the grading results presented here is that over 69000 participants' image sets have been graded in detail for characteristics of diabetic retinopathy. As the UKBB reaches more subjects for follow-up and linkage, this set of grading will become an important dataset for research purposes, and might provide ground-truth for further analysis. The dataset provides an unparalleled opportunity to examine eye disease in this cohort and will provide the much needed baseline data for the follow-up studies that are to be conducted by UKBB.

\section{Characteristics of Diabetic Retinopathy in the Funen Diabetes Database based on Latent Class Analysis}

M.B. Larsen ${ }^{1,2}$, M. Rowley ${ }^{3}$, J. Grauslund ',2, J.E. Henriksen ${ }^{5}$, A.C.C. Coolen ${ }^{3,4}$, T. Peto ${ }^{1,6}$

'Research Unit of Ophthalmology, Department of Clinical Research, Faculty of Health Science, University of Southern Denmark, Odense, Denmark

${ }^{2}$ Department of Ophthalmology, Odense University Hospital, Odense, Denmark

${ }^{3}$ Saddle Point Science, UK

${ }^{4}$ Department of Biophysics, Radboud University, Nijmegen, The Netherlands

${ }^{5}$ Steno Diabetes Center Odense, Odense University Hospital, Odense,

Denmark

${ }^{6}$ Centre for Public Health, Queen's University Belfast, Belfast, UK

DESIGN. Cross-sectional database study

PURPOSE. To evaluate the use of Bayesian latent class (BLC) modelling for analysing diabetic retinopathy (DR) screening data to capture known and potential novel associations with prevalent DR, allowing for correlations and dependence between time-to-event (TTE) proliferative DR.

METHODS. We extracted data from 14,453 patients with diabetes from the Funen Diabetes Database (FDDB) Denmark in 2014. BLC modelling was applied to data with DR severity grade represented by a time-to-event for each patient, to detect latent disease heterogeneity by identifying distinct patient sub-groups having different disease characteristics.

RESULTS. The number of patients with type 1 and 2 diabetes were 2,568 and 11,885 respectively. BLC analysis predicted two latent classes for both types of diabetes; the less frail class 1 represented long TTE (PDR), while the 
frailer class 2 were patients, who had high risk for proliferative DR after a relatively short duration of diabetes. In type 1 diabetes, $83.6 \%$ were assigned to class 2 . The average age and duration of diabetes in classes 1 and 2 were 53.9 versus 45.3 years, and 31.6 versus 19.1 years, respectively. Dominant associations in type 1 diabetes class 2 were duration of diabetes, $\mathrm{HbAlc}$, serum-creatinine and BMI. In type 2 diabetes, $99.5 \%$ were assigned to class 1 . Average duration of diabetes in classes 1 and 2 were 9.5 and 22.0 years. Dominant risk associations in type 2 diabetes class 2 were duration of diabetes, $\mathrm{HbAlc}$, events of hypoglycemia and serum-creatinine.

CONCLUSIONS. Latent class modelling showed biologically feasible results in both types of diabetes, using available ophthalmic and clinical data. Further analyses with either retro- or prospective data are needed to fully evaluate the use of latent class modelling to predict possible new and individualised risk factors for DR progression.

\section{Progression to Proliferative Diabetic Retinopathy in the Danish Registry of Diabetic Retinopathy}

S. Dinesen ${ }^{1,2}$, L. Stokholm ${ }^{2,3}$, T. Peto ${ }^{4}$, T. R. Savarimuthu ${ }^{5}$, J. Grauslund ${ }^{1,2,6}$, on behalf of the OASIS Study Group

'Department of Ophthalmology, Odense University Hospital ${ }^{2}$ Department of Clinical Research, University of Southern Denmark, Odense, Denmark

${ }^{3}$ OPEN - Open Patient data Explorative Network, Odense University Hospital, Odense, Denmark

${ }^{4}$ Centre for Public Health, Queens University Belfast, UK

${ }^{5}$ The Maersk Mc-Kinney Moller Institute, University of Southern Denmark, Odense, Denmark

${ }^{6}$ Steno Diabetes Center Odense, Odense University Hospital

DESIGN. Register based cohort study

PURPOSE. In Denmark, a national diabetic retinopathy (DR) screening programme has been implemented in order to detect sight-threatening disease such as proliferative diabetic retinopathy (PDR). In a national register-based cohort, we aim to identify demographic and clinical characteristics of patients, who later developed PDR, compared to patients who did not.

METHODS. We used the first screening episode as index date for patients with (cases) and without (controls) subsequent development of PDR. A multivariable Cox regression model was performed to measure the hazard ratio (HR) for incident PDR for all relevant demographic and clinical parameters.

RESULTS. A total of 147,841 patients were included in the study, of which 1,382 (0.935) subsequently developed PDR. Progression to PDR within 5 years occurred from ICDR-level 0 in 290 eyes $(0.2 \%)$, level 1 in 572 eyes ( 3.3 $\%)$, level 2 in 340 eyes (6.3\%), and level 3 in 180 eyes (20.3\%). HR for incident PDR were duration of diabetes (HR 7.64 per 10 years, $95 \%$ confidence interval [CI];
6.43-9.08), type 1 diabetes (HR 12.02, $95 \%$ CI; 10.05 14.39), Charlson Comorbidity score of 1 (HR 5.06, $95 \%$ CI; 4.36-5.87), 2 (HR, 2.04, $95 \%$ CI; 1.56-2.65) and 3 or more (HR, 3.36, $95 \% \mathrm{CI}$; 2.44-2.65), use of insulin (HR $10.05,95 \% \mathrm{CI} ; 8.38-12.05)$ and anti-hypertensiva (HR $1.96,95 \% \mathrm{CI} ; 1.60-2.41)$, and use of oral anti-diabetics (HR 0.21, $95 \%$ CI; 0.18-0.25). Compared to DR-level 0 , the risk of progression was increased with DR level 1 (HR 17.08, $95 \%$ CI; 14.31-20.39), level 2 (HR 33.07, $95 \%$ CI; 27.05-40.45) and level 3 (HR 98.95, $95 \%$ CI; 77.15-126.92).

CONCLUSIONS. Patients with subsequent progression to PDR differed from patients, who did not develop PDR by a variety of clinical and demographic characteristics.

\section{Inverse Cross-Sectional and Longitudinal Relations Between Diabetic Retinopathy and Obstructive Sleep Apnea in Type 2 Diabetes: Results from a National Screening Program}

J. Grauslund 1,2,3, L. Stokholm²,4, A.S. Thykjær ${ }^{1,2,3}$, S. Möller ${ }^{2,4}$, K.H. Rubin ${ }^{2,4}$, M.L. Rasmussen ${ }^{1,2}$, on behalf of the OASIS Study Group

'Department of Ophthalmology, Odense University Hospital, Odense, Denmark

${ }^{2}$ Department of Clinical Research, University of Southern Denmark, Odense, Denmark

${ }^{3}$ Steno Diabetes Center Odense, Odense University Hospital, Odense, Denmark

${ }^{4}$ OPEN - Open Patient data Explorative Network, Odense University

Hospital \& University of Southern Denmark, Odense, Denmark

DESIGN. Cross-sectional and 5-year longitudinal, registry-based, cohort study.

PURPOSE. In previous smaller studies, associations have been demonstrated between diabetic retinopathy (DR) and obstructive sleep apnea (OSA), but longitudinal relations have not been evaluated in larger cohorts. The aim of the present study was to assess the cross-sectional and prospective associations between DR and OSA in a national cohort of patients with type 2 diabetes.

METHODS. For cases, we included 153,238 patients with type 2 diabetes, who had attended diabetic eye screening and were registered in the Danish Registry of Diabetic Retinopathy (DiaBase). Each of these were matched by five control persons without diabetes of the same age and gender $(n=746,148)$. Data of exposure and outcome as well as systemic morbidity and use of medications were identified in national registers including DiaBase, the Danish National Patient Register, the Danish National Prescription Registry, and the Danish Civil Registration System. The index date was defined as the date of the first DR screening registered in DiaBase. Exposure was defined as present and level-specific DR, and main outcomes were crude, age- and sex-adjusted, and multivariable adjusted 
odds ratio (OR) for prevalent OSA as well as hazard ratios (HR) for 5-year incident OSA and DR.

RESULTS. Patients with type 2 diabetes were independently more likely to have prevalent OSA (OR 2.01, 95\% CI 1.95-2.08) and to develop OSA within five years (HR $1.55,95 \%$ CI 1.46-1.64). Type 2 diabetic patients with DR at baseline were less likely to have prevalent (OR 0.57, 95\% CI 0.52-0.62) and to develop incident OSA (HR 0.86, 95\% CI 0.74-0.99). Likewise, patients with OSA had a lower risk develop HR (HR 0.83, 95\% CI 0.74-0.92).

CONCLUSIONS. In a registry-based, national cohort study, patients with type 2 diabetes had a higher risk of OSA. However, a $43 \%$ decreased risk of prevalent OSA was demonstrated in patients with DR, and prospectively OSA and DR were both inversely related with each other.

\section{Incidence of Acute Angle Closure Glaucoma in the Northern Ireland Diabetic Eye Screening Programme (DESPNI) between 2016-202 I}

M. O’Donnell ${ }^{1,2}$, T. Peto ${ }^{1,2}$, A. Azuara-Blanco',2

'Centre of Public Health, Queen's University Belfast, UK

${ }^{2}$ Ophthalmology Department, Belfast Health \& Social Care Trust, UK

DESIGN. Clinical Audit

PURPOSE. This audit aims to determine the incidence of acute angle closure (AAC) glaucoma occurring within 72 hours of attendance at and having had a pupillary dilation with tropicamide as part of the Northern Ireland Diabetic Eye Screening Programme (DESPNI) between the dates of 01/09/2016 to $28 / 02 / 2021$.

METHODS. The Health and Social Care Trusts' clinical coding was used to identify all diagnoses of AAC and peripheral iridotomy procedures carried out in Northern Ireland between 01/09/2016-28/02/2021. These were subsequently cross-referenced DESPNI and NI Electronic Care Records to identify AAC within 72-hours of DESPNI attendance.

RESULTS. Altogether, 159 patients were identified as having had AAC in this time period. Of these, 27 had diabetes, most of which (26) attended DESPNI. However, only 1 of these patients had AAC within 72 hours of DESPNI's tropicamide dilation; the rest were all outside of this period, second closest was 10 day time-period. Over the 54 months period of observation, 206334 patients were seen in DESPNI; the dilation rate in NI is approximately $75 \%$, so 154750 patients were dilated. The incidence of ACC within the screening programme was calculated to be 1 event per 154750 episodes. This hypermetropic patient presented to eye casualty within 24 hours of dilation with tropicamide; he had AAC symptoms and unilateral reduced visual acuity. He has a history of Type 2 diabetes for two years. He had fluctuating vision in the left eye with some pain, worse in the dark, shallow angle and $24 \mathrm{mmHg}$ in the left eye. He was treated with pilocarpine $2 \%$ bd both eyes and was referred to Glaucoma clinic where he was diagnosed with ACC and had bilateral laser iridotomies.

CONCLUSIONS. ACC is a rare clinical emergency complication of mydriasis with tropicamide at DESP to improve image quality. It remains vital to advise patients of AAC symptoms and enable appropriate treatment. It is reassuring that our study improves our ability to inform patients of the low risk of angle closure within our service.

\section{Diabetic Retinopathy as a Potential Marker of Protection of Prevalent and Incident Migraine: Results from a National Cohort Study}

A. Vergmann ${ }^{1,3}$, L. Stokholm ${ }^{3}$, K.H. Rubin ${ }^{3}$, A.S. Thykjær ${ }^{1,2,4}$, J. Andresen ${ }^{5}$, J. Grauslund ${ }^{1,2,4}$, on behalf of the OASIS Study Group

'Department of Ophthalmology, Odense University Hospital, Odense, Denmark

${ }^{2}$ Department of Clinical Research, University of Southern Denmark, Odense, Denmark

${ }^{3}$ OPEN - Open Patient data Explorative Network, Odense University Hospital \& University of Southern Denmark, Odense, Denmark

${ }^{4}$ Steno Diabetes Center Odense, Odense University Hospital, Odense, Denmark

${ }^{5}$ Organization of Danish Practicing Ophthalmologists, Copenhagen, Denmark

DESIGN. This study was a national, register-based matched, cohort study

PURPOSE. This study aimed to examine diabetic retinopathy (DR) as a marker of prevalent and 5-year incident migraine in a national cohort of persons with diabetes in comparison with an age- and gender-matched group of non-diabetes persons.

METHODS. In a cross-sectional and 5-year prospective study, we included 205,970 persons (cases) with diabetes attending DR-screening registered in the Danish National Registry of Diabetic Retinopathy (DiaBase) and compared them to an age- and gender-matched group of 1,003,170 persons without diabetes (controls) from the Danish National Patient Registry. Data from DiaBase include the level of DR (0-4), graded according to the International Clinical Diabetic Retinopathy severity scale. Further data were collected from the Danish National Prescription Registry and the Danish Civil Registration System.

RESULTS. In the cross-sectional study, a multivariable model demonstrated a lower prevalence of migraine among patients with DR (OR 0.83, 95\% CI 0.81-0.85), with the lowest risk in those with DR level 1-4 (OR $0.69,95 \%$ CI $0.65-0.72$ ). Furthermore, the prevalence of migraine declined with increasing levels of DR (overall: 6.5\%, DR-levels; 0: 6.7\%, 1: 5.4\%, 2: 4.8\%, 3: 4.6\%, 4: $4.8 \%$ ). In the prospective study, a lower risk of incident migraine was found in a multivariable model among 
patients with diabetes (HR 0.76, 95\% CI 0.70-0.82), but this did not depend upon the presence of DR.

CONCLUSIONS. In a national study of more than 1.2 million persons, patients screened for DR had a lower risk of prevalent migraine. While increasing level of DR was associated with a lower prevalence of migraine in a cross-sectional study, DR was not identified as a protective marker of incident migraine over a course of five years in a prospective study.

\section{Diabetic Retinopathy and Chronic Respiratory Failure: A National Register-Based Cohort Study}

B. Thinggaard', J.R. Davidsen 2,3, M.E. Larsen 1,4, L.M. Stokholm ${ }^{1,4}$, J. Grauslund ${ }^{1,4,5}$, on behalf of the OASIS Study Group

'Department of Ophthalmology, Odense University Hospital, Odense, Denmark

${ }^{2}$ South Danish Center for Interstitial Lung Diseases (SCILS), Department of Respiratory Medicine, Odense University Hospital, Odense, Denmark ${ }^{3}$ Odense Respiratory Research Unit (ODIN), Department of Clinical Research, University of Southern Denmark, Odense, Denmark ${ }^{4}$ Department of Clinical Research, University of Southern Denmark, Odense, Denmark

${ }^{5}$ Steno Diabetes Center Odense, Odense University Hospital, Odense, Denmark

DESIGN. Registry-based, national cohort study.

PURPOSE. While diabetic retinopathy (DR) associates with retinal hypoxia, it is unknown whether DR is also linked to chronic respiratory failure (CRF). To explore if systemic hypoxia may also induce diabetic retinopathy (DR), we aimed to identify and characterize patients with $\mathrm{CRF}$ in a national DR screening program.

METHODS. In Denmark, DR screening is mandatory and results have been registered in the Danish Registry of Diabetic Retinopathy (DiaBase) database since 2013. We performed a national register-based cohort study of $1,209,140$ persons including 205,970 persons with diabetes and 1,003,170 age- and gender-matched controls. We estimated presence of CRF at the first screening episode (index date) and incidence of CRF during follow up. Charlson co-morbidity index was used to estimate individual co-morbidity burden using International Classification of Disease 10 codes from the Danish National Patient Registry.

RESULTS. Among patients with diabetes, we identified 1,980 persons with CRF $(0.96 \%)$ at the index date. In a multivariable regression analysis, the presence of CRF in persons with diabetes was higher compared to persons without diabetes (adjusted OR 1.52, 95\%CI 1.43-1.62), but the presence of CRF did not differ markedly between patients with DR level 0 (adjusted OR 1.53, 95\% CI 1.431.63) and levels grade 1-4 (adjusted OR 1.52, 95\% CI $1.30-1.78)$. During follow-up, we identified $1,726(0.84 \%)$ and 5,177 (0.52\%) events of CRF among persons with and without diabetes, respectively. Among patients with diabetes there was a positive correlation between the risk of developing CRF and increasing DR level (DR level 0: adjusted HR 1.16 95\% CI 1.09-1.25, DR-level 1-4: adjusted HR 1.69 95\% CI 1.48-1.93).

CONCLUSIONS. Patients with diabetes seem to have an increased risk to have and to develop CRF, where the risk rises along with increasing DR levels. Consequently, CRF may be a surrogate marker of DR. Increased attention of DR in CRF is warranted, and shorter DR screening intervals may be considered this patients category.

\section{Diabetic Retinopathy as a Potential Marker of Parkinsons Disease: Results from a Nationwide Register-Based Cohort Study}

M.E.C. Larsen ${ }^{1,2}$, A.S. Thykjaer ${ }^{1,2,3}$, F.N. Pedersen ${ }^{1,2}$, K.H. Rubin $^{2,4}$, M. Blaabjerg ${ }^{2,5}$, L. Stokholm ${ }^{2,4}$, J. Grauslund ${ }^{1,2,3}$, on behalf of the OASIS Study Group

'Department of Ophthalmology, Odense University Hospital, Odense, Denmark

${ }^{2}$ Department of Clinical Research, University of Southern Denmark, Odense, Denmark

${ }^{3}$ Steno Diabetes Center Odense, Odense University Hospital, Odense, Denmark

${ }^{4}$ OPEN - Open Patient data Explorative Network, Odense University Hospital \& University of Southern Denmark, Odense, Denmark

${ }^{5}$ Department of Neurology, Odense University Hospital, Odense, Denmark

DESIGN. Nationwide register-based cohort study PURPOSE. Evidence suggests that retinal neurodegeneration is an early event in the pathogenesis of diabetic retinopathy (DR). Given that the retina is an embryologically derived part of the brain, an association between DR and systemic neurodegeneration in Parkinson's disease (PD) has been proposed. In a national cohort, we aimed to investigate the association between DR and PD and to assess, if DR was a predictive 5-year marker of PD.

METHODS. Patients above 50 years of age who had attended the national DR-screening program and were registered in the Danish Registry of Diabetic Retinopathy between 2013 and $2018(n=173,568)$ were matched 1:5 by gender and birth year with a control population without diabetes $(n=843,781)$. At the date for the first DR-screening episode (index date), the risk of PD was compared between groups in a multivariable logistic regression model, and the subsequent risk to develop PD within five years was estimated in a multivariable cox proportional hazard model.

RESULTS. At the index date, the prevalence of PD was $0.28 \%$ and $0.44 \%$ among patients with and without diabetes, respectively. Patients with diabetes without DR were less likely to have PD (adjusted odds ratio [OR] 0.76, 95\% confidence interval [CI] $0.68-0.85$ ), but the protective factor disappeared in patients with DR levels 1-4 (OR 0.93, $95 \%$ CI $0.72-1.21$ ). In the prospective analysis, patients 
with diabetes were less likely to develop PD (adjusted hazard ratio [HR] $0.88,95 \%$ CI $0.78-1.00$ ), but this did not depend on the level of DR (DR-level 0: adjusted HR 0.91, 95\% CI 0.79-1.04, DR level 1-4: adjusted HR 0.77, 95\% CI 0.56-1.05).

CONCLUSIONS. In a national cohort followed for 3.2 million person-years, patients with diabetes were $21 \%$ and $12 \%$ less likely to have prevalent PD and develop PD in five years, respectively, compared to an age- and gendermatched population without diabetes. DR associated with a higher risk of prevalent PD, but did not affect the risk of 5-year incident PD.

\section{Diabetic Retinopathy and Risk of Major Depression: Bidirectional Results from a Danish Register-Based Nationwide Cohort Study}

F. Pedersen 1,2, F. Pouwer,2,3, L.M. Stokholm²,4, T. Peto ${ }^{2,5}$, J.

Grauslund ${ }^{1,2,3}$, on behalf of the OASIS Study Group

'Department of Ophthalmology, Odense University Hospital, Odense, Denmark

${ }^{2}$ Department of Clinical Research, University of Southern Denmark, Odense, Denmark

${ }^{3}$ Steno Diabetes Center Odense, Odense University Hospital, Odense, Denmark

${ }^{4}$ Open Patient data Explorative Network, Odense University Hospital \& University of Southern Denmark, Odense, Denmark

${ }^{5}$ Centre for Public Health, Queen's University Belfast, Belfast, UK

DESIGN. Matched register-based cohort study.

PURPOSE. Even though diabetes and microvascular complications are described as risk factors of depression, little is known regarding the interplay between diabetic retinopathy (DR) and depression. We aimed to investigate diabetes and DR as a marker of major depression along the inverse association.

METHODS. In Denmark, tax funded DR screening has been nationally implemented and results have mandatorily been registered in the Danish Registry of Diabetic Retinopathy (DiaBase) database since 2013.

We performed a national matched register-based cohort study of $1,209,140$ persons including 205,970 persons with diabetes and 958,170 age- and gender-matched persons without diabetes. Using International Classification of Disease 10 codes, major depression was ascertained by physician diagnostic codes of F32 (unipolar depression), F33 (recurrent depression) or F34.1 (dysthymia) from the Danish National Patient Register. We estimated presence of major depression at the index date and incident of major depression during follow up. In a secondary analysis, we investigated the inverse association in order to study whether major depression at index date was a risk factor of incident DR.

RESULTS. In a multivariable adjusted analysis, the presence of major depression was higher in persons with diabetes compared to persons without diabetes (adjusted OR $1.31,95 \%$ CI $1.28-1.34$ ), but the risk was lower in individuals with DR compared to persons without DR (adjusted OR 0.89 95\% CI 0.85-0.94). During follow-up, persons with diabetes were at higher risk of developing major depression (adjusted HR 1.27, 95\% CI 1.20-1.33), and persons with DR were at higher risk of major depression compared to persons with diabetes without DR (adjusted HR 1.14 95\% CI 1.04-1.26). We did not find an increased risk of DR incident in persons with diabetes and major depression (adjusted HR 0.96 95\% CI 0.89-1.04).

CONCLUSIONS. While the $11 \%$ lower prevalence of major depression in persons with DR could reflect a lower adherence to DR-screening in those with depression, the $14 \%$ higher 5 -year risk of major depression for persons with DR may indicate that DR is emotionally or pathophysiologically linked with development of depression.

\section{Disorganisation of Retinal Inner Layers (DRIL) in Participants with Diabetic Macula Oedema: The Relight Study}

C. Jamison', B. Hamill', A. Muldrew', K. Curran', T. Peto', U. Chakravarthy', RELIGHT STUDY GROUP²

'Centre for Public Health, Belfast Ophthalmic Reading Centre, Queen's University Belfast, UK

${ }^{2}$ Relight Study Group

DESIGN. Interventional clinical trial.

PURPOSE. To identify the change in disorganisation of the retinal inner (DRIL) in patients with diabetic macular oedema (DMO) treated with Ranibizumab in the RELIGHT Clinical Trial at baseline and 12 months.

METHODS. RELIGHT was a Phase 3B 18-months prospective multicentre single-arm study of individualised treatment with Ranibizumab in patients with DR in the United Kingdom. Diabetic retinopathy (DR) severity was established on colour fundus images using the Early Treatment of Diabetic Retinopathy Severity Scale (ETDRS). Optical Coherence Tomography (OCT) images were captured and graded for features of DR and presence, severity and extent of DRIL on the foveal scan. The three scans located immediately adjacent (superior and inferior) to the fovea were subjected to a graded categorical assessment of DRIL severity (none, mild, moderate and severe). For assessment of relationships between visual acuity and DRIL, a weighting system was devised with the foveal scan having the largest weighting (x 4) and the furthest scan (i.e., +3 or -3 ) having a weighting of $x 1$. Data were analysed in SPSS.

RESULTS. High quality OCT scans were available for 80 participants at baseline and 12-months. The mean (SD) age was $63 \pm 9$ years, and 23 participants $(28.7 \%)$ were women; median BCVA at baseline was 74.5 ETDRS 
letters. The mean weighted DRIL severity score was lower across all scans after treatment with Ranibizumab compared to baseline (12.9 vs 16.9). A higher frequency of more severe DRIL was observed at the fovea and the scans immediately adjacent. The mean DRIL at the fovea was $1365.5 \pm 784.6 \mu \mathrm{m}$ at baseline and $1013.2 \pm 687.0 \mu \mathrm{m}$ after 12 months post after treatment $(\mathrm{P}<0.001)$. The median DR severity of 43 remained the same throughout the study.

CONCLUSIONS. This study highlights that treatment with Ranibizumab results in improved retinal morphology as proven by the reduction of DRIL severity score after treatment of DMO. Further studies are required to ascertain how these changes might be reflected in functional vision tests.

\section{Risk of Progression of Type 2 Diabetes Individuals with Non-Proliferative Retinopathy: Characterization of Phenotypes in A I-Year Follow-Up Study}

L. Ribeiro',2, R. Coimbra', T. Santos', M.H. Madeira ${ }^{1,2}$, A.R. Santos $^{1,2,4}$, C. Lobo ${ }^{1,2,3}$, J. Cunha-Vaz ${ }^{1,2}$

'AIBILI - Association for Innovation and Biomedical Research on Light and Image, Coimbra, Portugal

${ }^{2}$ University of Coimbra, Coimbra Institute for Clinical and Biomedical Research (iCBR), Faculty of Medicine, Coimbra, Portugal

${ }^{3}$ Department of Ophthalmology, Centro Hospitalar e Universitário de Coimbra (CHUC), Portugal

${ }^{4}$ Department of Orthoptics, School of Health, Polytechnic of Porto, Porto, Portugal

DESIGN. To characterize the risks phenotypes (B and C) associated with diabetic retinopathy (DR), 141 type 2 diabetic (T2D) individuals with nonproliferative DR (ETDRS 20 to 47 ) were recruited ( 81 eyes phenotype B and 60 eyes phenotype $\mathrm{C}$ ) and followed for 1-year.

PURPOSE. DR phenotypes $\mathrm{B}$ and $\mathrm{C}$ has been shown to be associated with DR progression risk and vision-threatening complication development. In this study we aim to further characterize these phenotypes at systemically and at ocular levels.

METHODS. Of 141 individuals included, 135 completed the 1-year follow-up. Systemic evaluation included lipidic profile, interleukins (IL), C-reactive protein, haemoglobin Alc (HbAlc) levels and blood pressure. Ophthalmological examinations, performed at baseline and one-year, included visual acuity (BCVA), colour fundus photography (CFP) and optical coherence tomography (OCT and OCTA). Phenotype were classified at 6-month visit based on microaneurysm turnover (MAT, on CFP) and central retinal thickness (CRT, on OCT). Phenotype B identified by low MAT $(<6)$ and increased CRT; and Phenotype C identified by higher MAT ( $\geqslant 6$ ) with or without increased CRT. Vessel density (VD) was assessed by OCTA metrics in Superficial Capillary Plexus
(SCP), Deep Capillary Plexus (DCP) and Full Retina. Neurodegeneration was accessed by quantification of Ganglion Cell Layer+Inner Plexiform Layer (GCL+IPL) thickness on OCT. Differences between phenotypes were assessed by changes over one-year (Wilcoxon sign test).

RESULTS. During 1-year follow-up, and at systemic level, the study population showed significant lower levels of LDL cholesterol and increased HDL $(\mathrm{p}<0.001)$, and higher systolic pressure $(\mathrm{p}=0.038)$. Locally, ophthalmological evaluations revealed a decreased in VD in the DCP $(\mathrm{p}<0.001)$, increased BCVA (logmar scale $)(\mathrm{p}=0.007)$ and decrease in GCL+IPL thickness $(\mathrm{p}<0.001)$ and MAT $(\mathrm{p}<0.001)$.

When comparing phenotypes $\mathrm{B}$ and $\mathrm{C}$, the main significant differences observed were in systolic blood pressure $(\mathrm{p}=0.024), \mathrm{GCL}+\mathrm{IPL}$ thickness $(\mathrm{p}=0.016)$ and, as by definition, MAT $(\mathrm{p}<0.001)$.

A spearman correlation revealed moderate associations between VD, GCL+IPL Thickness and BCVA for phenotype $\mathrm{C}$, particularly at 12 -month visit.

CONCLUSIONS. In 1-year period of follow-up, only phenotype $\mathrm{C}$ show $\mathrm{DR}$ progression in neurogenerative changes (GCL+IPL thinning), which correlate with VD and visual acuity decrease, particularly apparent after 1 year.

\section{Comparison of Mean HbalC Value and EExcessa IC Index as Predictors of Retinopathy Development at Different Diabetes Duration}

A. Hirose ${ }^{1,2,3}$, Y. Maeda ${ }^{1,4}$, A. Goto ${ }^{5}$, M. Minami ${ }^{4}$, S. Kitano², Y. Uchigata $^{6}$

'MINAMI Diabetes Clinical Research Center, Japan

${ }^{2}$ Diabetes Ophthalmology, Diabetes Center, Tokyo Women's Medical University, Japan

${ }^{3}$ Ophthalmology, Graduate School of Medical Sciences, Kyushu University, Japan

${ }^{4}$ Clinic Masae Minami, Japan

${ }^{5}$ Graduate School of Data Science, Yokohama City University, Japan

${ }^{6}$ Medicine, Diabetes Center, Tokyo Women's Medical University, Japan

DESIGN. Retrospective cohort study

PURPOSE. We reported that using HbAlc (A1C) data for the total duration of type1 diabetes (T1D), mean A1C (mA1C) had a substantial capability to predict diabetic retinopathy (DR) development in our patients. This time, we compared the prediction capabilities of $\mathrm{mA} 1 \mathrm{C}$ and an index of $\Sigma$ excessA1C at different timepoints of duration in DCCT/EDIC database.

METHODS. We included 70 patients with no DR and a short duration of diabetes ( $\leqslant 14$ months) at DCCT baseline to examine only those who had A1C data for the total duration and DR data at all three timepoints (year5/9/13) after onset. The mean of yearly A1Cs from Nth to Xth year after onset was denoted as 
mA1Cyear(N-X). EexcessA1C calculated by adding all yearly $\mathrm{A} 1 \mathrm{C}$ values minus a threshold of 6.5 for each (\%: NGSP value; $48 \mathrm{mmol} / \mathrm{mol}$ ) from Nth to Xth year, was denoted as $\Sigma$ excessA1Cyear(N-X). These were actual values calculated from $\mathrm{A} 1 \mathrm{C}$ data of the full period of duration when $\mathrm{N}=1$, and simulated values, without using A1C data during ( $\mathrm{N}-1)$ years after onset, when $N \geqslant 2$. The retinopathy step of the final ETDRS severity scale at yearX was denoted as DRyearX. Retinopathy development, defined as positive when DRyear $X \geqslant 4$, was denoted as DRyearXdev+. We compared AUCs of ROC curves for DRyearXdev+ in mA1Cyear(N-X) and $\Sigma$ excessA1Cyear(N-X) between $\mathrm{N}=1$ and $\mathrm{N} \geqslant 2$ to study changes associated with an increase in the number of $N$ at $X=5 / 9 / 13$. Interactions by $X$ for correlations between mA1Cyear(1-X) or EexcessA1Cyear(1-X) and DRyearX were examined using ANCOVA. Two-sided $\mathrm{P}$ value $<0.05$ was considered significant.

RESULTS. AUCs of mA1Cyear(N-X) and LexcessA1C year(N-X) for DRyearXdev + were the same: $0.848(X=5)$, $0.876(X=9)$, and $0.821(X=13)$ indicating substantial capabilities when $\mathrm{N}=1$. But they tended to decline when $\mathrm{N} \geqslant 2$, and were significantly smaller at $X=9$ when $N \geqslant 5$, and at $X=13$ when $N \geqslant 7$. Interaction by $X$ was significant $(p<.001)$ for the correlation between mA1Cyear(1-X) and DRyearX, but not $(\mathrm{p}=0.139)$ for that between $\Sigma$ excessA1Cyear $(1-\mathrm{X})$ and DRyearX.

CONCLUSIONS. excessA1C may be superior to mA1C in predicting retinopathy development regardless of T1D duration, if we use $\mathrm{A} 1 \mathrm{C}$ data for as much of the total duration as possible.

\section{Subjective Visual Disturbances in Relation to Fluctuating Blood Glucose Levels in Adult Patients with Diabetes Mellitus: Results from a Pilot Study}

M.N. Rasmussen ${ }^{1,2}$, J. Grauslund ${ }^{1,2}$, M.J. Rothmann', M. Røder'

'Steno Diabetes Center Odense, Odense University Hospital, Denmark ${ }^{2}$ Department of Ophthalmology, Odense University Hospital, Denmark

DESIGN. Cross-sectional, questionnaire study.

PURPOSE. Major variation in blood glucose levels, may lead to visual disturbances with subsequent consequences like inability to work, loss of driving license, general frustrations and social isolation. Furthermore, in some patients this may increase fear of permanent visual loss. Patients often seek advice from optometrists but are often disappointed, given the inability to perform accurate refractive measurements in periods of impaired glycaemic control. The aim of this pilot study was to obtain a better understanding of the problem and to evaluate correlations between blood glucose levels and visual disturbances.
METHODS. An anonymous cross-sectional survey conducted at Steno Diabetes Center Odense, Odense University Hospital, Denmark. The questionnaire ware completed by patients attending retinopathy screening at the hospital between 1st of October 2019 and 26th of March 2021.

RESULTS. Subjective visual disturbances were evaluated in 173 patients. Of these, $126(72.8 \%)$ reported visual changes in relation to fluctuating blood glucose levels. Subgroup levels for visual changes were $71.0 \%$ of patients with long-standing diabetes who attended regular screening $(\mathrm{n}=155), 92.3 \%$ with newly diagnosed diabetes $(\mathrm{n}=13)$ and $80.0 \%$ who were pregnant $(\mathrm{n}=5)$. The vast majority of patients with self-reported visual difficulties in times of hyperglycaemia $(\mathrm{n}=56)$, reported blurry vision $(85.7 \%)$, and for those with hypoglycaemia $(n=63)$ mostly reported disturbances were flickering vision (38.1\%), blurry vision $(31.7 \%)$ and difficulties to accommodate (23.8\%). In patients with hyperglycaemia, visual disturbances were reported uniformly for all blood glucose levels between 10 and $25 \mathrm{mmol} / \mathrm{l}$, but in hypoglycaemia $80.1 \%$ of disturbances were reported in the range of $1.5-3.5 \mathrm{mmol} / \mathrm{l}$.

CONCLUSIONS. Subjective visual impairment is common in adult patients with diabetes, affecting almost three in four patients. Blurry vision is the most common disturbance in patients with hyperglycaemia, while flickering vision, blurry vision and accommodative problems are common during hypoglycaemia. The findings will be further explored in an upcoming study, which will include continuous glucose monitoring in combination with measurements of subjective and objective refractive parameters.

\section{Is Visual Function Altered in People with Uncomplicated Type 2 Diabetes or with Early Diabetic Retinopathy?}

K.J. Hellgren',2, D. Cox², C. Ball', A.C. Shore'2, K.M. Gooding ${ }^{2}$

'Department of Ophthalmology, Karlstad Hospital (Centralsjukhuset), Region Värmland, Karlstad, Sweden

${ }^{2}$ Diabetes and Vascular Medicine, University of Exeter Medical School and NIHR Exeter Clinical Research Facility, Royal Devon and Exeter NHS

Foundation Trust, UK

DESIGN. Cross-sectional observational study.

PURPOSE. To examine whether visual function is depressed in people with type 2 diabetes mellitus (T2DM) with either no clinical microvascular complications or with mild diabetic retinopathy (DR).

METHODS. Individuals with T2DM with either no evidence of clinical microvascular complications (micro/ macroalbuminuria, DR or neuropathy assessed by monofilaments) (DM group) or mild DR (defined as background DR, English Diabetic Retinopathy Grading Scheme) (DM+DR group) were identified from the Exeter SUMMIT cohort. Age matched participants without 
diabetes were also identified (non-DM group). Visual function was assessed by standard automated perimetry (SAP). Outcome measures were mean deviation (MD) and number of significantly depressed test points. Participants with other diseases likely to affect the visual field (e.g. glaucoma and cataracts) were excluded. Data from one eye per participant entered analysis. The number of depressed test points and MD values were compared across groups.

RESULTS. 169 participants were identified, with 51, 63 and 55 in the non-DM, DM and the DM+DR groups, respectively. Age was similar across these groups (mean (standard deviation): $68 \pm 9,70 \pm 7$ and $70 \pm 7$ years). The DM group had a higher number of depressed test points than the nonDM group (number of depressed points: DM group median (IQR) 2(5) vs non-DM group $0(3)$ test points, $\mathrm{p}=0.036$ ). Furthermore, the DM group had a lower MD than the non-DM group (MD: $0.19(1.96) \mathrm{dB}$ vs $0.61(1.63) \mathrm{dB}, \mathrm{p}=0.049)$. There was a non-significant trend for $\mathrm{MD}$ to be further depressed in $\mathrm{DM}+\mathrm{DR}$ group $(-0.33(2.63) \mathrm{dB})$ compared to the DM group ( $\mathrm{p}=0.092$ ), and the proportion of eyes with MD below normal limits were significantly higher in the DM+DR group than in the DM group ( $18 \%$ vs, $5 \%, \mathrm{p}=0.036)$.

CONCLUSIONS. Visual function, assessed by standard automated perimetry, is depressed in T2DM before any clinical microvascular complications are evident and further depressed when mild DR is present. The results suggests that visual function is altered by diabetes per se and future studies will investigate the relationship between T2DM, DR progression, particularly in the early stages, and visual function further.

\section{Long-Term Visual Outcomes and Morphologic Biomarkers of Vision Loss in Eyes With Diabetic Macular Edema Treated with Anti-Vegf Therapy}

E. Borrelli ${ }^{1,2}$, C. Barresi ${ }^{1,2}$, D. Grosso ${ }^{1,2}$, G. Lari ${ }^{1,2}$, R. Sacconi ${ }^{1,2}$, G. Querques ${ }^{1,2}$, F. Bandello,2

'Vita-Salute San Raffaele University Milan, Italy

${ }^{2}$ IRCCS San Raffaele Scientific Institute, Milan, Italy

DESIGN. Retrospective study.

PURPOSE. The aim of this study was to perform a qualitative and quantitative analysis on structural optical coherence tomography (OCT) images from diabetic macular edema (DME) eyes obtained more than 5 years after the initiation of anti-VEGF treatment to characterize morphologic characteristics correlating with good and poor longterm visual outcomes.

METHODS. Included subjects were required to have a longterm follow-up ( $\geqslant 5$ years) and evidence of resolved DME in at least one visit after 5 years of follow-up visits following the initiation of anti-VEGF therapy. The last visit with OCT evidence of resolved DME was considered as the study visit and was used for morphologic analysis. OCT images at the study visit were graded for qualitative features previously proposed as reflecting a distress of the neuroretina in patients with DME, as follows: (i) integrity of the ellipsoid zone (EZ) and external limiting membrane (ELM) bands within the foveola; (ii) integrity of the retinal pigment epithelium (RPE) within the foveola; (iii) presence of disorganization of retinal inner layers (DRIL) in the $1 \mathrm{~mm}$ central fovea. A quantitative topographical assessment of the inner and outer retinal thicknesses was also provided.

RESULTS. Sixty-one eyes (50 patients) were included and divided into two subgroups according to the visual acuity (VA) at the inclusion visit, yielding a group of 24 eyes with a VA $<20 / 40$ ("poor/intermediate vision" group), and 37 eyes with a $\mathrm{VA} \geqslant 20 / 40$ ("good vision" group). The ELM and RPE bands were more frequently disrupted/ absent in the "poor/intermediate vision" group $(\mathrm{P}=0.003$ and $\mathrm{P}=0.019$ ). Similarly, DRIL was more prevalent in the "poor/intermediate vision" group $(\mathrm{P}=0.013)$. The foveal and parafoveal outer retinal thicknesses were reduced in poor/intermediate vision eyes $(\mathrm{P}=0.022$ and $\mathrm{P}=0.044)$. Multivariate stepwise linear regression analysis demonstrated that the strongest associations with BCVA were with appearance of the RPE $(\mathrm{P}<0.0001)$, parafoveal outer retinal thickness $(\mathrm{P}=0.035)$, foveal outer retinal thickness $(\mathrm{P}=0.046)$, and appearance of the ELM $(\mathrm{P}=0.048)$.

CONCLUSIONS. This study provides OCT biomarkers associated with long-term visual outcomes in eyes with DME treated with anti-VEGF.

\section{Dry Eye Symptoms and Signs in Patients with Type 2 Diabetes}

\author{
S.A. Sandvik', A.E. Ystenæs', T.L. Morisbakk', V. Sundling' \\ 'National Centre for Optics, Vision and Eye Care, University of South-Eastern
} Norway

DESIGN. Cross-sectional study.

PURPOSE. To explore symptoms and signs of dry eye disease in patients with type 2 diabetes.

METHODS. Seventy-eight patients with type 2 diabetes $>$ 18 years consented to participate in the study. All underwent a full dry eye work-up according the Diagnostic Methodology of the Tear Film and Ocular Surface Society Dry Eye Workshop 11. Including the Ocular Surface Disease Index (OSDI) questionnaire, assessment of non-invasive break up time (NIBUT), tear osmolarity, fluorescein and lissamine green staining, Schirmer 1 test, and meibomian gland expressibility and quality. The study was approved by the Regional Committees for Medical Research Ethics (2018/04/REK sør-øst) in Norway. Data were analysed with Excel and IBM SPSS Statistics version 26 using standard statistical tests. A p-value $<0.05$ was considered statistically significant.

RESULTS. Fifteen patients (19.2\%) 95\% CI [11.2, 29.7] had dry eye symptoms (OSDI $\geqslant 13$ ). Signs of dry eye 
were found in 75 patients $(96 \%) 95 \%$ CI [89.2, 99.2]. In all, $72 \%, 68 \%, 59 \%$, and $51 \%$ patients had staining $>$ Oxford grade 1, Schirmer I test $<10 \mathrm{~mm}$, osmolarity $\geqslant$ $308 \mathrm{mOsm} / \mathrm{L}$ and NIBUT $<10$ seconds, respectively. Dry eye signs were not associated with dry eye symptoms, age or sex. All symptomatic patients had dry eye signs, $80 \%$ had evaporative and/or aqueous deficiency. Among asymptomatic patients with signs of dry eye disease, $85 \%$ had evaporative and/or aqueous deficiency.

CONCLUSIONS. In our study, patients with type 2 diabetes had dry eye symptoms despite dry eye signs and evaporative and/or aqueous tear film deficiency. This highlights the importance of assesing the tears and ocular surface in patients with type 2 diabetes to detect and manage dry eye disease and diabetic keratopathy

\section{The Statistician Will See You Now - How to Make the Best of a Scarce Resource}

\author{
I. Stratton ${ }^{1,2}$, K.I. Thorne ${ }^{2}$ \\ 'Gloucester Retinal Research Group, Gloucester Hospitals, Gloucestershire, UK \\ ${ }^{2}$ University of Southampton, Southampton, UK
}

DESIGN. A letter to the journal Diabetic Medicine from two medical statisticians

PURPOSE. Medical statisticians are a scarce resource and their time is precious. This is a piece of work to explain to clinicians how to communicate their ideas and questions to statisticians.

METHODS. Using combined expertise gathered over a total of over 50 person years working with clinicians we provide guidance on how best to use that 30 minute appointment in a statistics advice clinic.

RESULTS. Our list starts with the key piece of advice - consult a statistician before doing anything much at all. Being asked to do power calculations 24 hours before a grant submission is too late.

Explain what the condition is, who it affects, the disease course. Provide a list of acronyms and abbreviations. We need a lot of information about what you are measuring and how it is measured and how the data may vary. This might be influenced by the state of health of the person, the laboratory, the equipment, the person making the observation, the time of day the sample was taken, the weather and samples getting held up in the post. Sources of variation are important. Many of the observations made in ophthalmology are complex, the grading less than easy to understand. Diabetes is a complex health condition. Statisticians will need a careful explanation of what it is you are trying to find out. A rough idea of how many people you are able to recruit in the time you have available will mean that we can quickly tell you whether you have any chance of us being able to deliver a useful power calculation.
CONCLUSIONS. Statisticians do want to help - it might just seem at the time that we're being difficult. However, when we see that we have made a real difference to the risk of vision loss in people with diabetes we know that our work is worthwhile.

\section{The Retinal Oxygen Saturation can be used to Differentiate Retinal Neovascularisations from Intraretinal Microvascular Abnormalities (IRMAS) in Diabetic Retinopathy}

L. Petersen', T. Bek'

'Department of Ophthalmology, Aarhus University Hospital, Denmark

DESIGN. Prospective observational

PURPOSE. Diabetic retinopathy may be accompanied with the development of vascular abnormalities such as intraretinal microvascular abnormalities (IRMAs) and neovascularizations. The distinction between these two form of abnormalities is important since only the latter is immediately vision threatening and should be treated promptly. However, a distinction between the two types of vascular abnormalities may be difficult on the basis of regular fundus photography or fluorescein angiography alone. We conjectured that retinal oximetry might be used to differentiate the two types of vascular abnormalities in cases of doubt.

METHODS. Retinal oximetry was performed in 40 eyes with proliferative diabetic retinopathy and in 40 patients with severe nonproliferative diabetic retinopathy with IRMAs where the diagnosis had been made with certainty. The oxygen saturation in the vascular abnormalities were compared among the two groups.

RESULTS. The oxygen saturation in retinal neovascularizations was not significantly different from that in arterioles $(p=0.023)$, whereas the oxygen saturation of IRMAs was significantly lower than in arterioles $(p<0.0001)$ and higher than that in venules $(\mathrm{p}<0.0002)$.

CONCLUSIONS. Retinal oximetry can differentiate vascular abnormalities verified to be either neovascularizations or IRMAs. This suggests that the technique may be helpful in distinguishing the two types of vascular abnormalities when the distinction on the basis of fundus photography is uncertain.

\section{The Importance of Optical Coherence Tomography (OCT) for Identification of Clinically Significant Macular Oedema (CSMO) in UK Biobank (UKBB) \\ B. Hamill', T. Peto', NetwORC UK Graders², S. Madhusudhan ${ }^{3}$, K. Balaskas ${ }^{4}$, UK Biobank Eye and Vision Consortium ${ }^{5}$, P. Foster ${ }^{6}$}


'Centre for Public Health, Queen's University Belfast, UK

${ }^{2}$ NetwORC, UK

${ }^{3}$ St Paul's Eye Unit, Liverpool, UK

${ }^{4}$ Moorfields Eye Hospital NHS Foundation Trust, UK

${ }^{5}$ UK Biobank Eye and Vision, UK

${ }^{6}$ Insttitute of Ophthalmology, University College London, UK

DESIGN. UKBB is a biomedical database comprising lifestyle, biomedical and genetic data from over 500,000 UK participants aged 40-69. Almost 70000 participants had ophthalmic imaging of colour fundus photography and optical coherence tomography (OCT) at baseline.

PURPOSE. To provide human grading results on CSMO in UKBB participants and the importance of OCT scans to determine this.

METHODS. A single macula-centred colour image and OCT images were acquired using Topcon 3D OCT -1000 Mark II system for UKBB. Imaging took place on undilated eyes for UKBB. These images were analysed by trained and certified NetwORC UK reading centre graders in order to answer pre-determined questions for features of DR and CSMO. These data were captured in a study specific grading form linked to a study database. Quality assurance was carried out at a rate of 1:20 gradings.

RESULTS. Approximately $99 \%$ of colour fundus images and $99 \%$ of OCT images were available for both eyes. Of these, $90 \%$ of colour images and $99 \%$ of OCT images were gradable.

Diabetic related changes were identified in $714(1.04 \%)$ UKBB participants; 134 of these $(\sim 19 \%)$ were identified as having CSMO present. Of the 134 participants with CSMO, $37(\sim 28 \%)$ had maculopathy which was identified on OCT images only. Colour images did not show any sign of maculopathy in these instances.

CONCLUSIONS. These data illustrate the importance of the combined analysis of OCT scans with colour images for the accurate detection of CSMO in participants with diabetes. Without the use of OCT scans for identification of this parameter, approximately $28 \%$ of CSMO would have been missed in UKBB. Image quality was better overall on OCT scans compared to colour images and this may have been a factor here, as images were taken on undilated eyes, only one colour image was acquired and time allocation for ophthalmic imaging was limited. This research is particularly relevant for future epidemiological studies and DR screening programmes to ensure CSMO is identified accurately and efficiently, and to ensure it can be managed appropriately.

\section{Comparison of Different Metrics for the Identification of Capillary Closure in Diabetic Retinopathy Using OCTA}

I.P. Marques'1,2,3, L. Mendes', T. Santos', M.H. Madeira'1,2,3, J. Cunha-Vaz ${ }^{1,2,3}$
'AIBILI, Association for Innovation and Biomedical Research on Light and Image, Coimbra, Portugal

${ }^{2}$ Coimbra Institute for Clinical and Biomedical Research (iCBR), Faculty of Medicine, University of Coimbra, Coimbra, Portugal

${ }^{3}$ Center for Innovative Biomedicine and Biotechnology (CIBB), University of Coimbra, Coimbra, Portugal

DESIGN. Cross-sectional study

PURPOSE. To compare the performance of 34 different metrics computed based on optical coherence tomography angiography (OCTA) for identification of capillary closure (CC) in different DRRS groups.

METHODS. OCTA en-face slabs from 84 healthy eyes (70 \pm 4.8 years) and 78 eyes of diabetic individuals $(67 \pm 7.5$ years) were processed using different methods that include: abnormal intercapillary spaces (AIS), vessel density (VD) and 29 metrics extracted from the en-face slab after applying a Frangi filter.The ETDRS levels of the diabetic eyes included in our study range between 10 and 53. Data were acquired using CIRRUS HD-OCT 5000 with AngioPlex ${ }^{\circledR O C T A}$ (ZEISS, Dublin CA) using the acquisition protocol angiography $3 \times 3$. The AUC was used to rank the 34 metrics. All the metrics were computed based on the same en-face slab generated using the Carl Zeiss Meditec Density Exerciser (version:10.0.12787). This software was also used to calculate perfusion density (PD) and vessel density (VD).

RESULTS. The best separation between the eyes of individuals with type 2 diabetes in the initial stages of DR and the control group was obtained in the superficial capillary plexus (SCP), with the full retina (FR) also obtaining a competitive performance. The top five metrics for the SCP (and FR) are the AIS, the VD, FF_2, PD and FF_4. For all top 5 metrics, we found statistically significant differences for all the three ETDRS groups when compared with the age match healthy group. The AIS and VD show a progressive increase in CC on the ETDRS groups 10-20, 35 and 43-53. These results suggest that the percentage of the abnormal intercapillary spaces (AIS) and the number of vessels (VD) are the most discriminatory metrics. Furthermore, the vessels detected in the SCP and in the FR are similar which suggests that the main contribution of vessels that the AngioPlex detects comes from the SCP. CONCLUSIONS. The best separation between the eyes from diabetic individuals and the control group was obtained in the SCP. The results indicate that VD metric supported by the manufacturer is satisfactory. The values of these metrics on the ETDRS groups show a progressive increase in $\mathrm{CC}$ which correlate with the disease severity.

\section{Suspected Diabetic Retinopathy (DR) Related Changes In UK Biobank (UKBB)}

A. Sproule', T. Peto', NetwORC UK. Graders², S.

Madhusudhan $^{3}$, K. Balaskas ${ }^{4}$, UKBB. Eye and Vision

Consortium ${ }^{5}$, P. Foster ${ }^{6}$ 
'Centre for Public Health, Queen's University Belfast, UK ${ }^{2}$ NetwORC UK

${ }^{3}$ St Paul's Eye Unit, Liverpool, UK

${ }^{4}$ Moorfields Eye Hospital, NHS Foundation Trust, UK

${ }^{5} U K B B$ Eye and Vision Consortium, UK Biobank, UK

${ }^{6}$ Institute of Ophthalmology, University College London, UK

DESIGN. The colour and OCT images from the 70,000 UKBB volunteers (aged 40-69 years at baseline) provide a rich source of information for future linkage. The baseline UKBB images represent a subset of the 500,000 participants who volunteered for this population-based prospective study comprising lifestyle, biomedical and genetic data. PURPOSE. To provide human grading results for suspected DR related characteristics in the UKBB image set for those without characteristic features of haemorrhages and microaneurysms (HMAs) and understand the utility of OCT-based macular characteristics in this group.

METHODS. Topcon 3D OCT-1000 Mark II system was used to take undilated single macula centred colour and OCT images and subsequently analysed by trained and certified NetwORC UK graders in the Belfast, Liverpool and Moorfields Reading Centres. For the purposes of this study, patients without HMAs, but otherwise with typical features of DR including exudates, cotton wool spots, intra-retinal microvascular abnormalities and new vessels on colour images were included. Vessel tortuosity, venous beading and AV nipping were also recorded. Changes in OCT were evaluated for these patients and also for those where colours were ungradable but OCT was of good quality. Structured grading forms were used and data were analysed using descriptive statistics.

RESULTS. Isolated cotton wool spots and/or exudates were identified in 64 participants in UKBB. Characteristic vascular abnormalities were identified in 209 participants; 127 of whom had bilateral vascular changes suggestive of underlying systemic disease. The most common retinal vascular findings were vessel tortuosity in one or both eyes $(80 \%)$. There were 219 participants with inner retinal changes resembling diabetic eye disease such as hyper-reflective foci and layer disruption but with the distinct lack of features of retinopathy or any other major sight-threatening disease.

CONCLUSIONS. OCT images have revealed potentially significant number of additional patients who have suspected diabetes related changes but where these cannot be accurately confirmed due to either lack of HMAs or colour imaging. With the addition of OCT images, these UKBB participants have been correctly classified and been able to contribute to the final data analysis.

\section{Retinal Vessel Density, Vessel Diameter and Oxygen Saturation are Associated with Non- Proliferative Diabetic Retinopathy in Young Patients with Type I Diabetes}

N. Veiby', A. Simeunovic ${ }^{2,3,4,5}$, M. Heier ${ }^{3,5}$, M.C. Moe ${ }^{1,4}$, K. DahlJørgensen ${ }^{3,4,5}$, G. Petrovski ${ }^{1,4}$, H.D. Margeirsdottir ${ }^{3,5}$

'Center for Eye Research, Department of Ophthalmology, Oslo University Hospital, Oslo, Norway

${ }^{2}$ Department of Paediatrics and adolescent Medicine, Akershus University Hospital, Lorenskog, Norway

${ }^{3}$ Department of Paediatrics, Oslo University Hospital, Oslo, Norway

${ }^{4}$ Institute of Clinical Medicine, Faculty of Medicine, University of Oslo, Oslo,

Norway

${ }^{5}$ Oslo Diabetes Research Centre, Oslo, Norway

${ }^{6}$ Oslo Centre of Biostatistics and Epidemiology, Oslo University Hospital, Oslo, Norway

DESIGN. Cross sectional population study

PURPOSE. To determine the contribution of retinal vessel density, vessel diameter and oxygen saturation independently of other known risk factors in the development of non-proliferative diabetic retinopathy.

METHODS. Macular Optical Coherence Tomography Angiography (OCTA), central retinal artery/vein equivalent diameter (CRAE/CRVE) measurements and Retinal Oximetry were performed in a cross-sectional study of 166 eyes from 166 individuals with type 1 diabetes (T1D) aged 14-30 years. One hundred and twenty-one individuals had no retinopathy and 45 had non-proliferative retinopathy (33 had mild-, 10 had moderate- and 2 had severe). They were split in two groups, with and without retinopathy. Logistic regression analysis was used to investigate whether clinical well known risk factors and arteriolar-venular (AV) - difference in oxygen saturation, retinal vessel diameters and vessel density in the deep capillary plexus (VD-DCP) were associated with diabetic retinopathy.

RESULTS. Univariable logistic regression analysis showed that age, diabetes duration, higher waist circumference, decreased VD-DCP, CRVE and lower AV-oxygen saturation difference were significantly associated with diabetic retinopathy.

CONCLUSIONS. Our findings suggest that lower vessel density in the deep capillary plexus, wider central retinal venules and lower $\mathrm{AV}$-oxygen saturation difference are significantly associated with diabetic retinopathy. These ocular variables should be given appropriate weight in the risk stratification at early stages of the disease.

\section{Localization of Retinal Lesions in Diabetic Retinopathy in a Virtual Ocular Learning Platform (VIOLA)}

M.L. Rasmussen ${ }^{1,2}$, M.S. Hubel ${ }^{3}$, J.K.H. Andersen ${ }^{3,4}$, T.R. Savarimuthu ${ }^{3}$, J. Grauslund ${ }^{1,2,4}$

'Department of Ophthalmology, Odense University Hospital, Denmark ${ }^{2}$ Department of Clinical Research, University of Southern Denmark, Denmark ${ }^{3}$ SDU Robotics, The Maersk Mc-Kinney Moller Institute

${ }^{4}$ Steno Diabetes Center Odense, Odense University Hospital, Denmark 
DESIGN. Diagnostic study.

PURPOSE. In a virtual ocular learning platform (VIOLA) with marking exercises, heatmaps were constructed to evaluate the localization of diabetic retinopathy (DR)related lesions throughout DR-levels.

METHODS. In a training set of 200 retinal 45 degrees 6-field images with DR (International Clinical Diabetic Retinopathy Disease Severity Scale level 1-4), all DR-related lesions were independently marked by two retinal experts (JG and MLR). DR was evenly distributed across ICDR-levels $(n=50, n=49, n=51, n=50$ for DR-levels 1-4, respectively). Images were aligned with the optic disc located left. A template divided the images into 5 regions (upper, lower, nasal, temporal, and central). Heatmaps were formed for accumulated microaneurysms (MAs), haemorrhages (Hs), hard exudates (HEs), cotton wool spots (CWSs), intraretinal microvascular abnormities (IRMAs) and new vessels (NVs).

RESULTS. In all, 55,108 lesions were marked. For MAs, fewer lesions were located in the lower region throughout DR-level 1-4 (14.9\% in average compared to $22.0 \%$ (upper), $22.8 \%$ (temporal), $21.0 \%$ (nasal), and $19.3 \%$ (central). The same was true for Hs $(15.7 \%$ compared to $21.5 \%$ (upper), $23.0 \%$ (temporal), $22.0 \%$ (nasal), and $17.9 \%$ (central)). CWSs were more likely to be in the upper region for DR-levels 2-3 (32.0\% compared to $22.7 \%$ (temporal) and $22.4 \%$ (nasal)) and less likely to be in the lower (12.4\%) and central (10.5\%) region. However, for DR-level 4 , the majority $(41.6 \%)$ of the CWSs were located in the nasal region. HEs were mostly located in the central region $(67.8 \%)$ and most rarely in the lower (2.6\%). For DR-level 3, fewer IRMAs were located in the lower $(13.0 \%)$ and central (13.5\%) regions. Whereas for level 4, they were less likely located in the lower (13.3\%) and nasal $(15.2 \%)$ regions. Fewer NVs were found in the temporal (13.8\%) and central (6.1\%) regions, but were otherwise evenly distributed.

CONCLUSIONS. Overall, lesions were evenly distributed between the 5 regions throughout DR-levels. However, MAs, Hs, CWSs and IRMAs were less likely located in the lower region. Not surprisingly, HEs were mostly found in the central region. NVs were less likely found in the central and temporal regions.

\section{Agreement between Experts in the Detection of Diabetic Retinopathy Associated Lesions in a Virtual Ocular Learning Platform (VIOLA)}

M.S. Hubel', J.K.H. Andersen', M.L. Rasmussen ${ }^{2,3}$, T.R. Savarimuthu', J. Grauslund 2,3,4

\footnotetext{
'University of Southern Denmark, Denmark

${ }^{2}$ Department of Ophthalmology, Odense University Hospital, Denmark

${ }^{3}$ Department of Clinical Research, University of Southern Denmark, Denmark

${ }^{4}$ Steno Diabetes Center Odense, Odense University Hospital, Denmark
}

DESIGN. Diagnostic study.

PURPOSE. In order to establish a virtual ocular learning platform (VIOLA) with marking exercises, ground truth identification of specific diabetic retinopathy (DR) lesions were performed. The purpose of this study was to evaluate the lesion-specific consistency between experts in order to determine, if it was valid to use the common quantity between the experts for ground truth marking.

METHODS. In a training set of 300 retinal 45 degrees 6-field images, all DR-related lesions were independently marked by two retinal experts (JG and MLR). According to the International Clinical Diabetic Retinopathy Disease Severity Scale, we included retinal images at all DR-levels $(\mathrm{n}=100$, $\mathrm{n}=50, \mathrm{n}=49, \mathrm{n}=51, \mathrm{n}=50$ for DR-levels $0-4$, respectively). For each image, all DR-associated lesions were independently drawn by the two retinal experts. For all lesions, agreement between the graders was calculated by intraclass correlation coefficients (ICC) for a fixed set of two raters and defined as poor, moderate, good and excellent for ICC-values less than $0.5,0.50-0.75,0.75-0.90$, and above 0.90 , respectively.

RESULTS. For raters 1 and 2, the total number of DR-associated lesions marked in the images were 39,723 and 32,069. Corresponding numbers for specific lesions were 14,875 and 9,831 (microaneurysms), 3,621 and 3,401 (haemorrhages), 2,955 and 3,086 (hard exudates), 551 and 418 (cotton wool spots, CWS), 1,409 and 1,419 (intraretinal microvascular abnormalities, IRMA), 5,915 and 3.838 (new vessels), 99 and 192 (central laser) and 10,298 and 9,884 (panretinal laser, PRP). ICC values were 0.84 (microaneurysms), 0.83 (haemorrhages), 0.91 (hard exudates), 0.91 (CWS), 0.77 (IRMA), 0.07 (new vessels), 0.11 (central laser) and 0.99 (PRP).

CONCLUSIONS. While good or excellent agreement was demonstrated for expert-drawings of retinal microaneurysms, haemorrhages, hard exudates, CWS and PRP, poor agreement was demonstrated for new vessels and central laser. This indicates that the common quantity is a valid way to evaluate the former, while additional care should be taken for other DR-associated lesions.

\section{Optical Coherence Tomography (OCT) For Diabetic Maculopathy in a Digital Surveillance Clinic the Value of OCT in Digital Surveillance within Diabetic Eye Screening}

\author{
A. Farnsworth ${ }^{1,2}$, A. Wright ${ }^{1,2}$, H. Palmer', S. Jacob ${ }^{1,2,3}$ \\ 'University Hospitals Birmingham NHS Foundation Trust, Belfast, UK \\ ${ }^{2}$ Birmingham, Solihull and Black Country Diabetic Eye Screening Programme, \\ Birmingham, UK \\ ${ }^{3}$ Aston University, Aston, UK
}

DESIGN. Retrospective Audit

PURPOSE. To assess the impact of the recent introduction of OCT in one more of the digital surveillance 
clinics within the Birmingham, Solihull and Black Country Diabetic Eye Screening Programme.

METHODS. Between April 2019 and September 2019 an audit was undertaken retrospectively of patients attending Digital Surveillance. All had M1 grading on digital photography and OCT (Heidelberg Spectralis) Images were graded as maculopathy with or without oedema. Other lesions such as age-related macular degeneration (ARMD) and epiretinal membrane (ERM) were documented. Outcomes for those with maculopathy were either referral to Hospital Eye Service (HES) or followed up in the Digital Surveillance (DS) clinic with OCT.

RESULTS. 670 patients were included (M 374 F 300 U 23; mean age 60yrs). Ethnicity was not recorded reliably but was predominantly White, Afro/Caribbean, Asian. OCT images were adequate and gradable in 654 patients (97.6\%). 139 (20.7\%) reverted to annual recall. 433 (64\%) were kept within DS. $5(0.75 \%)$ patients were excluded as they were unsuitable for screening. $83(12.4 \%)$ patients were referred to HES, 48 with macular oedema (mean $400 \mu \mathrm{m}$ range 319 to 625 , SD 54.8) who remained in HES for treatment and review. $6(0.9 \%)$ patients were referred to HES/GP for other eye pathology (wet ARMD/ glaucoma). $4(0.60 \%)$ patients were referred to slit-lamp because images were unassessable.

CONCLUSIONS. OCT is effective in DS clinics, providing a definitive diagnosis and measure of macular oedema. This study showed that only a small proportion of patients with maculopathy needed HES referral. All referrals to HES were deemed appropriate and stayed in HES for management. Identification of false positive M1 can continue annual recall safely. OCT allows appropriate prioritising of patients needing treatment more urgently. It has helped ease the pressure in HES with major capacity issues especially during the recent Covid pandemic.

\section{Detection of Diabetic Retinopathy Lesions Using Deep Learning Based Semantic Segmentation in Retinal 45 Degrees 6-Field Images}

\author{
J. Andersen 1,2, M.L. Rasmussen ${ }^{3,4}$, M.S. Hubel', J. Grauslund 2,3,4, \\ T.R. Savarimuthu' \\ 'SDU Robotics, The Maersk Mc-Kinney Moller Institute, University of \\ Southern Denmark \\ ${ }^{2}$ Steno Diabetes Center Odense, Odense University Hospital, Denmark \\ ${ }^{3}$ Department of Ophthalmology, Odense University Hospital, Denmark \\ ${ }^{4}$ Department of Clinical Research, University of Southern Denmark, Denmark
}

DESIGN. Diagnostic study

PURPOSE. A deep learning model was trained to perform segmentation of diabetic retinopathy (DR) lesions in order to detect these. While deep learning models for classification of DR as well as segmentation of some lesions such as hemorrhages and microaneurysms have been studied, segmentation and detection of intraretinal microvascular abnormalities and retinal new vessels are lesser explored uses.

METHODS. The model was trained on 240, 45 degrees 6-field retinal images of 6528 by 6528 pixels with 31,061 semantic labels for microaneurysms (MA, $\mathrm{n}=17,957)$, haemorrhages $(\mathrm{n}=5361)$, hard exudates (HE, $\mathrm{n}=4789)$, cotton wool spots (CWS, $\mathrm{n}=680$ ), intraretinal microvascular abnormalities (IRMA, $\mathrm{n}=1882$ ) and retinal new vessels $(\mathrm{NV}, \mathrm{n}=392)$, independently annotated by two retinal experts. The model was based on the U-net encoder-decoder architecture using a pre-trained encoder with residual connections and multiple neurons with different kernel sizes in the output segmentation layer. The model was trained using an instance weighted categorical cross-entropy loss. The model was evaluated on 30 images, using the markings of Expert 1 as gold standard and those of Expert 2 as comparator.

RESULTS. For detection of DR lesions, the network achieved sensitivities of 0.63 (MA), 0.74 (haemorrhages), 0.70 (HE), 0.43 (IRMA), 0.36 (CWS), 0.59 (NV) and precisions of 0.71 (MA), 0.46 (haemorrhages), 0.61 (HE), 0.56 (IRMA), 0.73 (CWS) and 0.29 (NV).For the inter-grader comparative analysis, sensitivities were 0.40 (MA), 0.57 (haemorrhages), 0.83 (HE), 0.42 (IRMA), 0.56 (CWS), $0.51(\mathrm{NV})$, and precision was $0.78(\mathrm{MA}), 0.61$ (haemorrhages), 0.74 (HE), 0.54 (IRMA), 0.65 (CWS), 0.37 (NV). CONCLUSIONS. The network achieves higher sensitivity for MA detection with slightly lower precision. For haemorrhages, sensitivity was also higher but precision was noticeably lower. For IRMA, performance was about equal for both metrics, while the higher sensitivity for NV yields somewhat lower precision, although this was relatively low for both. For HE, both sensitivity and specificity was significantly lower, while detection of CWS had a low sensitivity, but a higher precision.

\section{Testing The Performance of Risk Prediction Models to Determine Progression to Referable Diabetic Retinopathy (RDR) in an Irish Type 2 Diabetes Cohort}

\author{
J. Smith', N. Lois', P.H. Scanlon², I. Stratton², D. Wright ${ }^{3}$ \\ 'Wellcome Wolfson Institute for Experimental Medicine, School of Medicine, \\ Dentistry and Biomedical Sciences, Queens University Belfast, Belfast, UK \\ ${ }^{2}$ Gloucestershire Hospitals NHS Foundation Trust, Gloucestershire, UK \\ ${ }^{3}$ Belfast Association for the Blind, School of Medicine, Dentistry and \\ Biomedical Sciences, Queens University Belfast, Belfast, UK
}

DESIGN. Validation of prognostic models on patient data from country external to where models were developed. PURPOSE. Prediction models developed to determine risk of progression to referable diabetic retinopathy were tested in an Irish cohort of people with type 2 diabetes 
METHODS. A cohort of 939 people with T2D followed prospectively was used to test the performance of risk prediction models developed in Gloucester, United Kingdom, and Iceland. Observed risk of progression to RDR in the Irish cohort was compared with that derived from each of the prediction models evaluated. Receiver operating characteristic (ROC) curves assessed models' performance.

RESULTS. The original Gloucester model, which includes results of two consecutive retinal screenings; a model incorporating, in addition, systemic biomarkers [HbAlc and serum cholesterol]; and a model including results of one retinopathy screening, $\mathrm{HbAlc}$, total cholesterol and duration of diabetes, had acceptable discriminatory power (area under the curve (AUC of 0.69, 0.76 and 0.77, respectively). The Icelandic model, which combined retinopathy grading, duration and type of diabetes, $\mathrm{HbA} 1 \mathrm{c}$ and systolic blood pressure, performed very similarly (AUC of 0.74 ).

CONCLUSIONS. In an Irish cohort of people with T2D, the prediction models tested had an acceptable performance identifying those at risk of progression to RDR. These risk models would be useful in establishing more personalised screening intervals for people with T2D.

\section{Comparing Siva and Vampire for Vascular Geometric Findings in Patients with Type I Diabetes}

S. Dabbah', D.M. Møller'2, T. Peto³, J. Grauslund ${ }^{1,2,4}$, M.L. Rasmussen ${ }^{1,2}$

'Department of Ophthalmology, Odense University Hospital, Odense, Denmark

${ }^{2}$ The Clinical Research Institute, University of Southern Denmark, Odense, Denmark

${ }^{3}$ Centre for Public Health, Queen's University Belfast, UK

${ }^{4}$ Steno Diabetes Centre Odense, Odense University Hospital, Odense, Denmark

DESIGN. Comparison Study

PURPOSE. To compare two semi-automated software applicants for vascular geometry assessment in patients with type 1 diabetes (T1DM) - Singapore I Vessel Assessment (SIVA) and Vascular Assessment and Measurement Platform for Images of the REtina (VAMPIRE) - and to examine these parameters in associations with proliferative diabetic retinopathy (DR).

METHODS. A cohort of 185 patients with T1DM participated in a clinical examination in 2011, where retinal images were taken. DR was graded using the Early Treatment Diabetic Retinopathy Study (ETDRS) score. Proliferative DR (PDR) was defined as ETDRS level 61 or above. Disc-centered images were analyzed using SIVA and VAMPIRE and needed adjustments were made by trained graders. Central retinal artery and vein equivalent (CRAE and CRVE), Fractal dimension and arterial and venular tortuosity were measured and outcomes from the two applicants were compared using Pearson correlation coefficient. Mann-Whitneys test were performed to investigate correlations between PDR and retinal vascular parameters.

RESULTS. In 4 patients images were non-gradable for the programs; hence 181 images were used for analysis. Mean age and duration of diabetes was 37 years and 29.5 years, respectively, and $49.2 \%$ were male. DR severity was as follows: no DR (ETDRS 10: 2.2\%), non-proliferative DR (ETDRS 20-53: 67.8\%), and PDR (ETDRS $\geqslant 61: 30.0 \%$ ). There was a high correlation between outcomes from SIVA and VAMPIRE for CRAE, CRVE and fractal dimension (Pearson's r: 0.89, 0.89 and 0.72, respectively). However, correlations for arterial and venular tortuosity data were low (Pearson's r: 0.27 and 0.17, respectively). Patients with PDR had lower fractal dimension compared to patients with no DR and non-proliferative in both software applicants (SIVA: 1.37 vs. $1.44, \mathrm{p}<0.001$ and VAMPIRE: 1361.26 vs. $1396.77, \mathrm{p}=0.002$ ). No other vascular parameters were significantly associated to PDR.

CONCLUSIONS. We found a high correlation between the two software applicants for vascular caliber and fractal dimension, as opposed to low correlations for tortuositymeasurements. Fractal dimension was the only parameter that was significantly correlated to PDR.

\section{Children Living with Diabetes See and Thrive with Al Review: Childstar}

N. Whitestone', K. Curran' ${ }^{2}$ N. Congdon ${ }^{1,2}$, T. Peto ${ }^{2}$, J.L. Patnaik $^{1,4}$, N. Jaccard ${ }^{1}$

'Orbis International, USA

${ }^{2}$ Centre for Public Health, Queen's University Belfast, UK

${ }^{3}$ Orbis Bangladesh, Dhaka, Bangladesh

${ }^{4}$ Department of Ophthalmology, University of Colorado School of Medicine, USA

DESIGN. Retrospective study

PURPOSE. Background: Diabetic retinopathy (DR) is a common complication of diabetes, and if left un-treated, can lead to visual impairment and blindness. The prevalence of diabetes in children and young adults is rising rapidly worldwide. The role of artificial intelligence (AI) in the detection of DR appears promising, and the accuracy of AI systems to detect DR has been extensively studied in adult patients; however, there are very limited studies in children. Orbis International's Cybersight AI provides automated recommendations for DR staging from fundus photographs based on Deep Learning algorithms, which were trained using data from adult patients $(18+$ years $)$.

Objective: To compare the clinical performance of the Cybersight AI DR grading capabilities with human graders on a dataset of children and young adult fundus photographs. 
METHODS. Between May 2016 and February 2018, children and young adults were screened at Dhaka (BIRDEM-2) hospital, Bangladesh in Orbis' child-focused DR project, funded by USAID. Fundus images were captured using a digital non-mydriatic camera. Two screening cut-offs were considered: 1) any DR detection (R1 or more severe based on UK NHS grading scheme), and 2) referable DR detection (R2 or more severe). For both scenarios, AI screening accuracy vs certified UK NHS graders was calculated at a patient level.

RESULTS. Fundus images from 1256 children and young adults (3-26 years) were interpreted, including 200 individuals $(16 \%)$ with any DR and 15 individuals $(1 \%)$ with referable DR. When comparing Cybersight $\mathrm{AI}$ and human graders, the area under the receiver operating characteristic curve (AUROC) was $88 \%$ and $97 \%$ for detection of any DR and referable DR, respectively.

CONCLUSIONS. Cybersight AI performed well on images from children and young adults despite its algorithms being trained on adult eyes. AI may be an effective tool to screen children with diabetes to identify referable DR, and can help to reduce demands on scarce physician resources in low-resource settings.

\section{Creating Artificially Training Data for Al Detection of Retinal Microaneurysms}

S.L.B. Sørensen', J.K.H. Andersen', J. Grauslund², T.R. Savarimuthu'

'The Maersk Mc-Kinney Moller Institute, SDU Robotics, University of Southern Denmark, Denmark

${ }^{2}$ Department of Ophthalmology, Odense University Hospital, Odense, Denmark

DESIGN. Grounded theory

PURPOSE. We develop a method of creating artificial training data containing microaneurysms for AI segmentation model development without additional manual annotations. Medical data are often unbalanced towards healthy cases. This will impact the training of medical artificial intelligence models, as fewer samples with pathology will make the model biased towards the normal cases and prevent the models to reach their potential.

METHODS. Given an image of a healthy retina and a mask of positions, in which one wants MAs placed, the method can generate an image that complies with the mask by placing realistic MAs. We trained three different networks to achieve this:

-An inpainting model was trained to create new MAs from a given mask. This was done with $68564 \times 64$ patches with MAs and their mask taken from 88 1-field retina images from the E-Ophtha MA retina dataset.

-A UNet cascade model was trained to create a heatmap where MAs can be placed in retina images. The model contains a secondary UNet that predicts a mask for the vessels and optic nerve.

-An inpainting model was trained to inpaint healthy eyes. This model's training data was $100 \mathrm{k}$ patches from the retina images without MAs. The model was used to remove the MAs from the retinal images. We used these images together with the original masks to train the placement model From the output of the placement model, random MA placements can be sampled. Together with a randomly chosen mask from one of the 685 patches, new MAs can be inpainted in a healthy eye using the inpainting model.

RESULTS. The sick to healthy inpainting model was combined with the placement model to create artificially training data for a MA detection model. The detection model achieved a sensitivity of 0.47 with only 2 false positives per image on real data.

CONCLUSIONS. With multiple models, it was possible to create retinal images with MA from healthy retina images. The placement model can also be used with other methods of placing MA with known position creating unlimited ground truth data for training AI networks.

\section{Performance Evaluation of Software-Assisted Diabetic Retinopathy Screening}

T. Nissen 1,3,4, P. Vestergaard ${ }^{2,3}$, K.C. Schielke', M.M. Dawidowicz', J. Grauslund ${ }^{4}$, K. Aasbjerg' ${ }^{5}$, H. Vorum'

'Department of Ophthalmology, Aalborg University Hospital, Denmark ${ }^{2}$ Department of Endocrinology, Aalborg University Hospital, Denmark ${ }^{3}$ Steno Diabetes Center, North Jutland, Denmark

${ }^{4}$ Department of Ophthalmology, Odense University Hospital, Denmark

${ }^{5}$ Himmerland Eye Clinic, Denmark

DESIGN. Comparative study to evaluate the performance of a support vector machine learning model (RetinaLyze System A/S) for binary classification of the presence or absence of diabetic retinopathy (DR).

PURPOSE. The growing number of patients with diabetes worldwide increases the need for DR-screening. Traditional screening is labour-intensive and requires special trained personnel. This can be quite a monotonous activity, and keeping focus and performance through a work day can be toiling. The purpose of the study is to evaluate the sensitivity and specificity of a commercially available DR screening tool and compare the result to a majority decision of three retinal specialists.

METHODS. We included five fundus images of 1001 patients (1001 eyes) with diabetes, which had previously been captured during normal screening procedures. First, the images were graded using the software. Then, three certified retinal specialist from two hospitals independently and blinded graded the images according to the International Clinical Diabetic Retinopathy Disease 
Severity Scale. A majority decision among the specialists was used for the final label. A power calculation was made by a statistician indicated that a minimum of 960 eyes were to be included.

RESULTS. After the exclusion of 12 ungradable eyes, 989 eyes were included. Prevalence of DR 58.7\% (retinal specialists) and $77.2 \%$ (software). As compared to retinal specialists, sensitivity, specificity, positive predictive value and negative predictive value of the software was $97.0 \%$, $49.5 \%, 73.2 \%$ and $92.2 \%$, respectively. Furthermore, the software detection of DR included $57.0 \%$ true positives, $20.8 \%$ false positives, $1.7 \%$ false negatives, and $20.4 \%$ true negatives. General agreement between the retinal experts was $76.5 \%$, and individual eye-label consensual scores between the retinal experts were $77.0 \%, 78.0 \%$ and $76.0 \%$.

CONCLUSIONS. The software was tested on 1001 eyes labelled by majority decision by three retinal specialist. The software showed a high sensitivity, but limited specificity. As a screening tool for DR, the software can be useful in exclusion of the presence of DR. This may decrease the amount of images with potential DR needed to be manually reviewed.

\section{Pretraining on Abstract Modules on a Virtual Reality Vitreoretinal Simulator is a Waste of Time}

S.B. Petersen 1,2,4 A.H. Vestergaard ${ }^{1,2}$, A.S. Thomsen ${ }^{3,4}$, L. Konge $^{4}$, M. La Cour ${ }^{3}$, J. Grauslund 1,2, A.S. Vergmann ${ }^{1,5}$

'Department of Ophthalmology, Odense University Hospital, Odense, Denmark

${ }^{2}$ Department of Clinical Research, Faculty of Health Science, University of Southern Denmark, Odense, Denmark

${ }^{3}$ Department of Ophthalmology, Rigshospitalet-Glostrup, Glostrup, Denmark ${ }^{4}$ Copenhagen Academy for Medical Education and Simulation, Centre for HR, Copenhagen, Denmark

${ }^{5}$ Regional Center for Technical Simulation, Region of Southern Denmark, Odense Denmark

DESIGN. Prospective, randomised, controlled, two-centre study.

PURPOSE. Virtual reality vitreoretinal simulation training is a learning tool for aspiring surgeons to master complex eye surgeries such as vitrectomy on patients with diabetes in case of vitreous haemorrhage or retinal traction detachment. To optimize efficacy, it is important to consider the potential beneficial effect of abstract training elements. Thus, we aimed to study the inter-transferability of skills in the training program, by investigating whether pretraining with abstract modules in virtual vitreoretinal surgery affected the performance-curve positively, when proceeding to procedure-specific modules.

METHODS. This study investigated a validated virtual reality vitreoretinal training program on the EyeSi Surgical
Simulator consisting of two abstract and two procedurespecific modules. Medical students were randomised into two groups: Group 1 pretrained with the abstract modules until they reached performance-curve plateau. Hereafter, both groups (1 and 2) trained with the procedure-specific modules until they reached performance-curve plateau. Plateau was defined as three consecutive sessions with the same score with an acceptable variation. The primary outcome was time used to reach performance-curve plateau in the procedure-specific modules.

RESULTS. A total of 68 medical students were included, and equally randomised into two groups. Group 1 used a median time of 88 minutes to reach plateau in the abstract modules. Thereafter, the participants in Group 1 and 2 did not differ in time to reach plateau in the procedure-specific modules (183 minutes versus 210 minutes, $p=0.40$ ) or in the amplitude of plateau (ILMP3 81 points versus 79 points, $\mathrm{p}=0.68$, and PostH 386 points versus 74 points, $\mathrm{p}=0.14)$. Group 1 and 2 differed significantly in the starting score of ILM peeling level 3 (0 points versus 3.5 points, $\mathrm{p}=0.03$ ).

CONCLUSIONS. We were not able to show positive skill transfer from the abstract modules to the procedure-specific modules. Neither in time, starting score, or amplitude of plateau. Thus, the results raise the question whether all modules of the training program are necessary. Therefore, we cannot recommend the implementation of abstract modules in the curriculum for aspiring vitreoretinal surgeons.

\section{Vitrectomy for Sight-Threatening Diabetic Retinopathy in Denmark 1999-2019: A National, Registry-Based Study}

T.L. Torp ${ }^{1,2}$, L. Stokholm ${ }^{2,5}$, A.S. Thykjær ${ }^{1,2}$, K.C. Schielke, N. Andersen ${ }^{4}$, J. Grauslund ${ }^{1,2,3}$, on behalf of the OASIS Study Group

'Department of Ophthalmology, Odense University Hospital, Odense, Denmark

${ }^{2}$ Department of Clinical Research, University of Southern Denmark, Odense, Denmark

${ }^{3}$ Steno Diabetes Center Odense, Odense University Hospital, Denmark ${ }^{4}$ Organization of Danish Ophthalmologists, Copenhagen, Denmark ${ }^{5}$ OPEN - Open Patient data Explorative Network, Odense University Hospital \& University of Southern Denmark, Odense, Denmark

${ }^{6}$ Department of Ophthalmology, Aalborg University Hospital, Aalborg, Denmark

DESIGN. National, prospective cohort study PURPOSE. In diabetic retinopathy (DR), vitrectomy is indicated for non-clearing vitreous haemorrhage or tractional retinal detachment. In this registry-based study, we investigated the 20-year risk of diabetic vitrectomy in patients with diabetes, who had attended DR-screening and were registered in a national quality database. 
METHODS. We included Danish patients screened for DR between 2013-2018, who were mandatorily registered in the Danish Registry for Diabetic Retinopathy (DiaBase). Data regarding diabetic vitrectomy was extracted from the Danish National Patient Register if a specific operative (vitrectomy: CKD65) and diagnostic (diabetic retinopathy: H360, OR vitreous haemorrhage: H431, OR tractional retinal detachment: H334B) coding was registered. We excluded patients with diabetic vitrectomy before 1999 and those in whom diabetes was diagnosed more than 12 months after diabetic vitrectomy.

RESULTS. Among 205,970 patients registered in DiaBase, 2,622 (1.3\%) were registered with at least one episode of diabetic vitrectomy during 1999-2019. Multivariable adjusted odds ratios (OR) with $95 \%$ confidence intervals (CI) for diabetic vitrectomy were male gender (OR 1.09, 95\% CI 1.01-1.19), younger age (ORs $0.75,0.58,0.58,0.48$ and 0.33 for increasing 10 -year age-groups as compared to 18-39 years), type 1 diabetes (OR 1.30, 95\% CI 1.10-1.54), duration of diabetes (ORs 1.12 and 1.18 per 10 years for patients with type 1 and 2 diabetes, respectively), marital status (OR 1.31, 95\% CI 1.17-1.46, for patients never married), Charlsons comorbidity score (ORs 2.64, 1.96 and 1.75 for patients with increasing scores), use of insulin (OR 2.09, 95\% CI 1.81-2.42), antihypertensive therapy (OR 2.45, 95\% CI 2.14-2.80) and level of DR at first screening episode (ORs 4.91, 7.11, 14.2 and 71.0 for DR-levels 1-4, respectively).

CONCLUSIONS. In a national cohort including all diabetic vitrectomies in Denmark in a 20 -year span, vitrectomy was performed in $1.3 \%$ of patients and was more likely to occur in younger, unmarried male patients with type 1 diabetes. DR-level at the first screening episode was identified as the principal risk factor.

\section{Treating Diabetic Macular Oedema: I 2-Weeks Intervals in the First Year}

Q. Wen, T. Peto, D.M. Wright

Centre for Public Health, Queen's University Belfast, UK

DESIGN. Historical cohort data

PURPOSE. Treatment of diabetic macular oedema (DMO) with intravitreal anti-vascular endothelial growth factors (anti-VEGF) injections places a substantial burden on the healthcare system, patients and society. We determined the percentage and characteristics of patients whose injection interval could be extended to $\geqslant 12$ weeks in the first treatment year. Patients attended the Mater Macula Service DMO Clinics, Belfast Health and Social Care Trust (BHSCT), between 05/2008-03/2021.
METHODS. METHODS: Clinic attendances and injection data were recorded in a structured format using an electronic medical record system (Medisoft, UK). Data were downloaded and anonymised at source once Caldicott Guardian permission was granted. Inclusion criteria was "Those $\geqslant 18$ years of age having attended the BHSCT Macula Clinic at least once during the period of 05/2008-03/2021 with a diagnosis of diabetic retinopathy (DR)/DMO." The only exclusion was applied to test patients.

RESULTS. Altogether, 4330 patients (2649 male, $61.2 \% ; 1681$ female, $38.8 \%)$, age $21-99.8$ years old (median 64.8) were included in the extracted dataset with 20141 records of anti-VEGF injections. The unit of analysis was the eye so criteria were applied at the eye level. 2215 eyes received at least one anti-VEGF injection with 269 eyes having received only one therefore excluded from the interval analysis. A total of 1946 eyes from 1324 patients (808 males, 61.0\%; 516 females, $39.0 \%$ ) with a total of 17926 treatment intervals analysed. Altogether $22.7 \%$ received bilateral injections. Mean visual acuity (VA) of ETDRS letters was $60.7 \pm 17.4 ; 7.5 \%$ had less VA than 33 letters and $26.2 \%$ had over 73 letters. Altogether, $52.9 \%$ of the patients had at least one treatment interval of at least 12 weeks in the first year of treatment, of these, $61.4 \%$ were males, proportional to the gender composition of the whole cohort (proportion test $\mathrm{p}=0.79$ ).

CONCLUSIONS. The substantial treatment burden of anti-VEGF injections is demonstrated in this cohort. Over half of the DMO patients had at least one interval of 12 weeks or over in the first year of treatment and so analysis of this cohort will help understand how best manage these patients.

\section{Functional and Structural Efficacy of a Novel Combinational Therapy of Aflibercept and Timely Focal/Grid Photocoagulation in Diabetic Macular Edema: Do Clinical Study Results Compare Favorably with a Standard-Of-Care Treated Real-Life Population?}

M.M. Jørgensen ${ }^{1,2}$, A.H. Vestergaard ${ }^{1,2}$, S.L. Blindbæk², T. Peto ${ }^{1,4}$, J. Grauslund ${ }^{1,2,3}$

'Department of Clinical Research, Faculty of Health Sciences, University of Southern Denmark, Denmark

${ }^{2}$ Department of Ophthalmology, Odense University Hospital, Denmark

${ }^{3}$ Steno Diabetes Center Odense, Odense University Hospital, Denmark

${ }^{4}$ Centre for Public Health, Queen's University Belfast, UK

DESIGN. Retrospective and registry based study. PURPOSE. To evaluate treatment efficacy of diabetic macular edema (DME) comparing a study population receiving intravitreal inhibition of vascular endothelial 
growth factor (VEGF) and post-loading focal/grid photocoagulation with a matched, real-life population receiving standard of care treatment.

METHODS. In this 12-months study, 43 eyes of 32 patients from the published study "Aflibercept and navigated versus conventional laser in diabetic macular oedema: a 12-month randomized clinical trial" were included along with 46 eyes from 38 standard of care patients constituting a real-life population. In the study population, patients had received a loading dose of three monthly aflibercept injections followed by focal/ grid photocoagulation and pro re nata (PRN) aflibercept injections. In the real-life population, patients received standard of care treatment with PRN VEGF-inhibition and focal/grid photocoagulation at the discretion of the treating physician. Principal measurements for the study were numbers of intravitreal injections, best corrected visual acuity (BCVA) and central retinal thickness (CRT). RESULTS. At baseline, no differences in age, sex and type of diabetes were observed between the two groups, but the study population had had diabetes for fewer years than in the real-life group ( 12.0 vs. 19.9 years, $p=0.02)$. At month 12 , patients in the study group had received fewer intravitreal aflibercept injections (4.4 vs. 5.9, p $<0.01)$ and were more likely only to receive the three mandatory injections in the loading phase $(39.5 \%$ vs. $13.0 \%$, $\mathrm{p}=0.01)$. There was no difference in visual gains $(+8.4$ vs. +5.8 ETDRS letters, $\mathrm{p}=0.19)$ nor CRT-reduction between the two groups (-97.4 vs. $-103.7 \mu \mathrm{m}, \mathrm{p}=0.78)$.

CONCLUSIONS. In comparison to a matched, reallife DME-population, patients in predefined combined treatment with intravitreal aflibercept and post-loading focal/grid photocoagulation received $25 \%$ fewer intravitreal injections and were almost three times as likely to avoid further treatment after initial loading and photocoagulation.
Assessment of Complications during the Late Postoperative Period after Drainage Surgery of Secondary Neovascular Glaucoma in Patients With Diabetes Mellitus

V.A. Biletskaya ${ }^{1,2}$, D.V. Lipatov',2, M.A. Frolov'

'RUDN University, Moscow, Russia

${ }^{2}$ Endocrinology Research Centre, Moscow, Russia

DESIGN. Retrospective analysis.

PURPOSE. Assessment of patients with diabetes mellitus (DM) in the late (10-15 years after surgery) postoperative period after neovascular glaucoma (NG) drainage surgery by analysing the effectiveness of intervention (study of the dynamics of intraocular pressure, presence or absence of pain and various eye complications).

METHODS. 146 patients (150 eyes) with NG and DM were operated on the base of the National Endocrinology Center (Moscow, Russia) between 2006-2020. All patients had anti-glaucoma drainage surgery with implantation of Ahmed valves (138 eyes) and Molteno valves (12 eyes). The analysis of long-term (10-15 years) postoperative complications was carried out for 59 patients.

RESULTS. No patient had pain in early and/or late postoperative period. In 10-15 years after surgery, corneal vascularisation was diagnosed in $25.4 \%$ of cases (15 patients), epithelial-endothelial corneal dystrophy in $20.3 \%$ of cases (12 patients), eyeball atrophy in $13.5 \%$ (8 patients) and 3 patients $(5.1 \%)$ had enucleation with prosthetics. The cause in case of corneal vascular cataract and epithelial-endothelial dystrophy is DM as primary disease.

CONCLUSIONS. Despite the presence of serious postoperative complications, drainage surgery for DM patients continues to be an effective method of surgical treatment of patients with secondary uncompensated NG for controlling pain. 